UNITED STATES DEPARTMENT OF THE INTERIOR

GEOLOGICAL SURUEY

ISOPLOTZOO

A PLOTTING AND REGRESSION PROGRAM FOR ISOTOPE GEOCHEMISTS, FOR USE WITH HP SERIES 200 COMPUTERS

by

K. R. LUDWIG

OPEN-FILE REPORT $85-513$

This report is preliminary and has not been reviewed for conformity with U.S. Geological Survey editorial standards and nomenclature. Any use of trade names is for descriptive purposes only, and does not imply endorsement by the U.S. Geological Survey. 
SUBJECT

PAGE

Introduction - 1

Hardware required by ISOPLOT200

Preliminary Remarks on Using ISOPLOT200

Getting Started - 4

Loading the Program -

Setting up the Plot -

Choosing the Axis Names -

Entering the Plotbox Limits

Defining the Physical Size of the Plot

Centered Plots -

Uncentered Plots -

Entering the Plot Limits

Plotting Data Points - 9

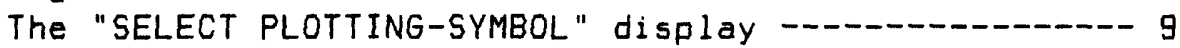

Polygon Plotting-Symbol

Plotting Symbols that Indicate Errors

Entering Data from the Keyboard -

The ADD Screen -

Labelling Words or Phrases on the Plot

Storing and Retrieving Data from Data Files -

Accessing Data Files from ISOPLOT200

Using Data from Data Files -

Using Keyboard-Entered Data as a Temporary File --16

Yorkfits of Data

Yorkfit "MODELS" -

Obtaining isochron ages from Yorkfit 1 ines -.-.-.-- 19

Re-using Yorkfit points; plotting error-envelopes ---- 19

Selecting a Dashed or Solid Yorkfit Line - 20

The OPTIONS Screen

Creating Concordia Plots 21

Obtaining Growth Curves for Pb-Isotope Plots

Dumping a CRT Plot to the Plotter or Printer - 22

References - 23

Figure Captions -

Figures -

Appendix: Program List of ISOPLOT200 29 


\section{ISOPLOT200 - A PLOTTING AND REGRESSION PROGRAM FOR ISOTOPE GEOCHEMISTS, FOR USE WITH HP SERIES 200 COMPUTERS}

by

Kenneth R. Ludwig

\section{INTRODUCTION}

ISOPLOT200 is a program designed primarily for use by isotope geologists, but of value for anyone who wants to plot $X-Y$ data in a simple, rapid, and flexible manner. Data can be entered either from the keyboard or from datafiles produced by the commercially-available VISICALC program. Data can be plotted as a wide variety of symbols, including various error-symbols and polygons. ISOPLOT200 can regress data using a modification of the most widely-used two-error regression algorithm, and will calculate isochron ages or concordia-intercept ages directly from the regression lines. U-Pb isotope concordia plots (together with intercepts and errors of regression lines) are a built-in feature, as are single-stage growth-curves for Pb-isotope plots. ISOPLOT200 is intended to be simple to use, and 50 is provided with HELP screens, extensive error-trapping, and detailed prompts. As a result, most users will seldom need to use this manual.

The pedigree of ISOPLOT200 extends back several years, starting from programs for HP-9830/9831 computers (Ludwig, 1979a, 1979b, 1982). The most recent precursors are a group of programs written for HP-86/87 computers (Ludwig, 1983). Because of the much greater computational speed of the HP Series 200 computers, together with a much more powerful BASIC language superset and greater memory, it seemed worthwhile to develop a much more userfriendly version that would take advantage of the hardware advances. Hence ISOPLOT200.

\section{HARDWARE REQUIRED BY ISOPLOT200}

ISOPLOT200 was written for Hewlett-Packard Series 200 computers (Models 216, 217, 236, 9816, 9817, 9836). The required BASIC language version is either BASIC 2.0 or BASIC 3.0 (I've tried only the RAM-based versions), with the necessary language extensions varying according to the version (see below). You should have at least 750 kilobytes of RAM (before loading the BASIC language). The program requires an HPIB printer (at address 701 ) and an HP-7475A 6-pen plotter (at address 705). For the 
BASIC 2.0 language, you must also load (using the LOAD BIN command) the two binary programs AP2_1 and GRAPH2_1, sold by HP as language extensions. For the BASIC 3.0 language, the following binary language-extension programs are required: GRAPH, GRAPHX, MAT, CLOCK, KBD, ERR, KNB2_0, CS80, DISC, HPIB, and MS.

The program assumes that the keyboard is the OPTION 805 (ASCII extended keyboard character set) keyboard -- the one that is identical to the standard keyboard of the HP-9836. I assume that the standard Series 200 keyboard would work with little or no modification, but haven't actually tried one myself.

The programs were developed using a dual single-sided $3.5^{\prime \prime}$ disk drive (model HP-9121), and should work equally well with the HP82905B 5.25" disk drive. The program itself is compatible with the HP-9122 double-sided 3.5" disk drive: however, HP has not made the UISICALC program available for the double-sided disk drives, nor will they continue selling UISICALC for the Series 200 at all. You can't even get a version of UISICALC for the HP9121 copied onto disk that can be read by the HP-9122 drive. So for the present, if you use the program with the HP-9122 doublesided 3.5" disk drive, you'll have no way of constructing datafiles that ISOPLOT200 can read - unless you want to write a program to mimic the VISICALC storage format yourself. Data files created by PBDAT200, a Pb-U-Th isotope data-reduction program (Ludwig, in press) are compatible without recourse to UISICALC, however.

I've looked at CONTEXT MBA, a combination spreadsheet/word processing/file management/communications program that's supposed to be compatible with the HP-9122. Unfortunately, the documentation is opaque and the program itself much too complex for easy use, so I'm not sure whether I'll ever have the time to modify ISOPLOT200 to use CONTEXT MBA spreadsheet datafiles. Or whether any users would bother with it either.

\section{PRELIMINARY REMARKS ON USING ISOPLOT200}

First, you should understand some of the basic features of the computer -- the keyboard editing keys (left, right, up, and down arrows), what the softkeys ( $k 0-k 9$ ) are for, and how to answer a query by the program. So if you have the time, read the introductory manual to the computer. 


\section{GETTING STARTED}

\section{LOADING THE PROGRAM}

To load the program (the BASIC language and required extensions must aready be in memory, of course), just put the ISOPLOT200 disk in the default drive (the one that lights up when you type CAT, then press EXECUTE), type in LOAD "ISOPLOT", 1 and wait about a minute. The program will start itself.

SETTING UP THE PLOT (X-Y plots)

The first CRT-display of the program (the one that appears when you press the RUN key) will look something like this:

Rev. July 5, 1985 K.R. Ludwig, U.S. Geol. Survey

$\langle\langle\langle\langle\langle\langle\langle\langle\langle<$ ISOCHRON or $X-Y$ PLOTTER $\gg \gg \gg\rangle \gg\rangle) \gg\rangle\rangle$

CRT PLOT

CRT OUTPUT

Press CTRL C for concordia plot

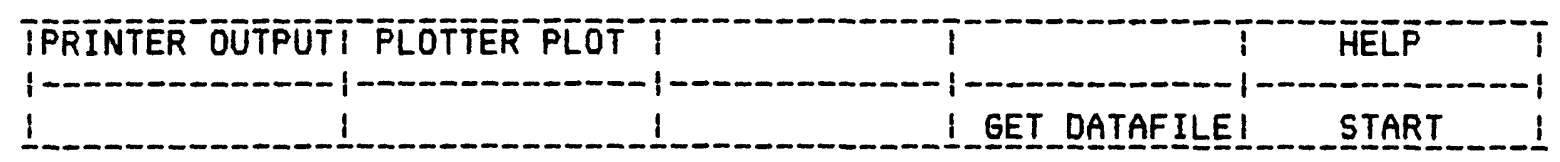

In this section, I'll discuss the use of the ISOCHRON or $X-Y$ PLOTTER. If the display says CONCQRDIA PLOTTER at the top, press CTRL I. If the display says PLOTTER PLOT at the upper left, press $k 1$, and if the display says PRINTER OUTPUT at the upper right, press ko.

First, select where your plot and your printed output are to be sent. Softkey kO determines whether the printed output is to be sent to the printer or the CRT, and toggles between the two. Look at the upper right of the CRT to see what the current status is. Notice that the kO label will always be the opposite. This is because the softkey labels indicate what the softkey will do when pressed, not what the current status is. 
Next, select where you want the plot to appear -- on the CRT or the plotter. The current status is indicated at the upper left of the CRT, and is changed (toggles from CRT to plotter) by pressing $k 1$.

If you select the plotter as the plotting device, several more softkeys will be labelled on the bottom of the CRT, as shown below:

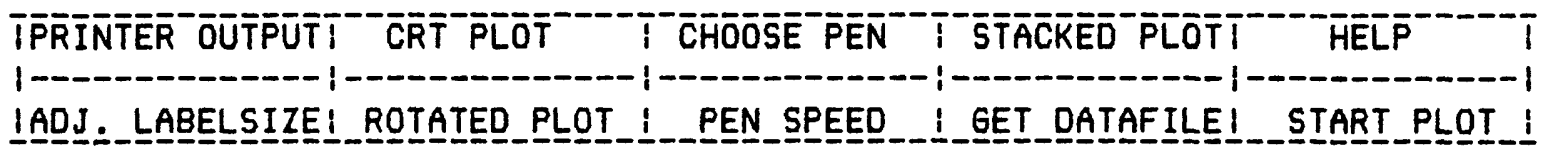

To select one of the 6 pens of the HP-7475A plotter, press k2. One of the bottom lines of the CRT will then ask you to choose a pen.

To select a pen speed (how fast the pen moves while drawing lines), press k7. Use the fastest speed (10) for draft plots, slower for final or publication quality plots. The default speed is the fastest.

To vary the relative sizes of the axis and tick labels of the plot, press $k 5$. The default size (1) is suitable for plots that will not be reduced, but larger sizes are best for plots that will be made into $35 \mathrm{~mm}$ transparancies or will be significantly reduced during reproduction.

If you select either a slow pen-speed or large labels, ISOPLOT200 will draw each part of the plot twice (slightly of fset the second time to give thicker lines) to improve its clarity.

If you want the plot to be drawn vertically on the paper instead of horizontally, press k6 (ROTATED PLOT).

If you want to stack plots $5 Q$ that they share the same $X-$ axis (and $X$ limits), construct the lower plot as usual, but with specifically-defined limits for the plot-box (see below). Then for the upper plot, press k3 (STACKED PLOT). Position the plotbox precisely over the lower box. Because you specified a STACKED PLOT, the upper plot will be drawn without labels for the $X$-axis or the $X$-axis ticks, and without the first $Y$-axis ticklabel. 


\section{Choosing the Axis Names:}

When you've finished selecting the physical parameters of the plot with the softkeys, as described above, press the START softkey ( $k 9$ ). The CRT display will then be:

...Use n to start superscripts or end subscripts,

* to start subscripts or end superseripts

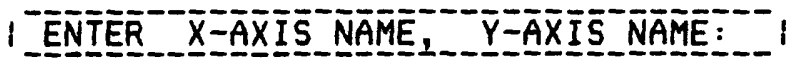

(press CONTINUE to use PPM Sr and PPM Rb)

Shorthand for common axis-names:

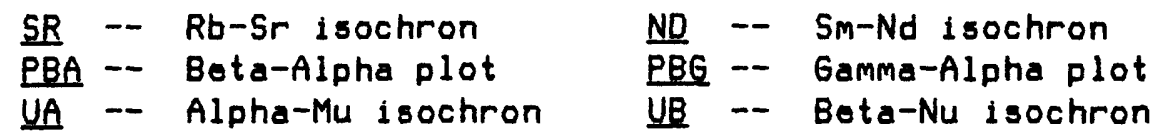

The axis names are the names that will be labelled under the $X$-axis and to the left of the $Y$-axis. You can respond to the query in 3 different ways: ( 1 ) by typing in the $X$-axis name and the $Y$-axis name (separated by a comma) and pressing the ENTER key, (2) by pressing CONTINUE to re-use whatever names were used in the last plot (PPM $5 r$ and PPM Rb in the above example), or (3) entering one of the "shorthand" codes indicated in the screen: SR to obtain the names for an Rb-Sr isochron plot, PBA for a ${ }^{206} \mathrm{~Pb} /{ }^{204} \mathrm{~Pb}-{ }^{207} \mathrm{~Pb}-204 \mathrm{~Pb}$ plot, and so on.

You can specify superscripts or subscripts in the axis names by using the "symbol to start superscripts (or cancel subscripts), and the * symbol to start subscripts (or cancel superscripts. So if you wanted the axis names looking like

$$
{ }^{87} \mathrm{Sr} /{ }^{86} \mathrm{Sr} \text { and } \mathrm{Al}_{2} \mathrm{O}_{3}
$$

you would enter the names this way:

$$
{ }^{\wedge} 87 * 5 r / \wedge 86 * 5 r \quad \text { and } A 1 * 2 \wedge 0 * 3
$$




\section{Entering the Plotbox Limits:}

After you've entered the axis names for the plot, the CRT will display:

\section{ENTER $X$ AND $Y$ LIMITS $\left(\left[X_{\min },\right] X_{\max },\left[Y_{m i n},\right] \quad Y_{\max }\right) ?$}

(press CONTINUE to re-use $0,14, .7,1.2$ )

Your response to this query defines what the minimum and maximum $X$ and $Y$ values of the plotbox will be. You can enter just 2 values, in which case the $X$ and $Y$ minimum values will be assumed to be zero, or 4 values if at least one of the minimum limits is nonzero. If you just want the same limits as were used for the last plot, though, just press CONTINUE without entering any values. At this point, if your plot is to be drawn on the CRT, the computer will construct the plot box, tick-labels, and axis labels.

The format for entering values in response to a query by ISOPLOT200 is similar to the above example throughout the program. Values for prompts in brackets are optional -- a default value will be used if you don't enter a number for them. To enter several values at once (as indicated in the prompt), just separate the values by commas. And, for many queries, there is a default value or set of values that will be assumed if you just press the CONTINUE key without typing in a specific response.

Defining the Physical Size of the Plot:

If you requested that your plot be drawn on the plotter (rather than the CRT), you'll have to specify the size of the plot. The CRT query will be:

$$
\text { ENTER PLOT-SIZE }(3-10) ?
$$

(Press CONTINUE for default size of 8 )

(Press k4 for nonstandard dimensions/locations) 


\section{Centered plots:}

If you want a plot with a "normal" height-to-width ratio (about the same as an 8.5 " by 11 " sheet of paper) and centered in the paper, enter a number from 3 to 10 in response to the above query. A value of 10 gives the largest plot that will fit on a $8.5^{\prime \prime}$ by $11^{\prime \prime}$ sheet of paper, and a value of 3 a plot only $30 \%$ as large. Intermediate values give proportionately intermediate sizes. The default size is 8 .

\section{Uncentered plots:}

If you want a plot with a different height-to-width ratio (perhaps only $5 \mathrm{~cm}$ high by $10 \mathrm{~cm}$ wide), or located off-center in the paper, press softkey $k 4$. The CRT query will then be:

PRESS KQ TO DEFINE THE PLOT-LIMITS FOR THE ENTIRE FIGURE AREA, K4 TO DEFINE LIMITS FOR THE PLOT-BOX ONLY

If you want to define the area that the total plot will occupy, including tick-labels and axis names, press $k \theta$. If you want to specify the location and area of the plot in terms of the plot box, however (the plot box is the box outlining the area where the data points are actually plotted), press $k 4$. The latter option is particularly useful for cases where you are putting two or more plots on a single sheet of paper, and essential if you want to stack two plots that share the same $X$-axis.

\section{Entering the plot limits:}

Once you've selected how you want to define the location and size of the plot, the CRT will query:

$$
\begin{aligned}
& \text { ENTER PLOT-LIMITS IN mm: X-MIN, X-MAX, Y-MIN, Y-MAX? } \\
& \text { (max. limits are } 0-249[X] \text { and } 0-179.5[Y], \\
& \text { lower-left corner of paper is } 0,0)
\end{aligned}
$$

Enter 4 values, separated by commas, to define the location of the lower-left and lower-right limits of the plot (X-MIN, XMAX), and the upper-left and upper-right limits of the plot ( $Y$ MIN, $Y$-MAX). The lower-left corner of the paper is defined as $X=0, \quad Y=0$. Enter your values in millimeters. The computer will now start constructing your plot. 


\section{PLOTIING DATA-POINTS}

\section{THE SELECT PLOTTING-SYMBOL DISPLAY}

After ISOPLOT200 has drawn the plot box, tick-labels and axis-names, you're ready to start plotting your data points. First, though, you must select a plotting symbol. The CRT display will be:

SELECT A PLOTTING SYMBOL TO PLOT DATA

press CTRL L to draft a phrase on the plot

press CTRLP to dump plot to printer

press CTRL D to dump plot to plotter

press CTRL H for HELP

press any key for keyboard plotting-symbol

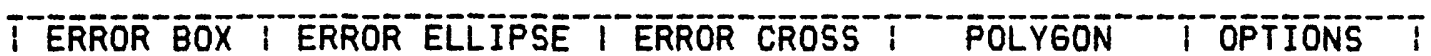 \\ $1-\ldots 1-1-1-1,-1$ \\ SOLID_LINE!_CHOOSE_PEN_ YORKFIT__GET_DATAFILE_INEW_PLOT_I}

I'll refer to this disply as the "SELECT PLOTTING SYMBOL" display. The bottom rows show the softkey definitions.

You can do several things from this part of the program besides just selecting a plotting symbol, but for now we'll ignore them. The simplest type of plotting symbol to select is just an alphanumeric one - that is, using one of the keyboard symbols, such as $+, *, X, O$, or $A, H,-5, \#$. To select a keyboard symbol, just press that key, and the computer will go on to ask you for the data itself.

POLYGON PLOTTING-SYMBOL

You can use a polygon for a plotting symbol, with any number of sides, size, and rotational orientation, either open or filled. Press the softkey labelled POLYGON (unshifted for an open polygon, shifted for a filled polygon). The CRT will then query: 
POLYGON-SYMBOL: ENTER \#SIDES (- for star), [,SIZE (mM) [, ROTATION F(O-360]]

\section{Press CONIINUE to re-use last values $(20,2.5,0)$}

As usual, the parameters in brackets represent optional ones, so you can enter 1, 2, or 3 values (or press CONTINUE to re-use the last values entered). The number of sides can vary from 3 (a triangle) to a large number (in effect, giving a circle). The SIZE parameter refers to the diameter of the polygon, in millimeters. Default is $2.5 \mathrm{~mm}$. The ROTATION parameter refers to the angular orientation (counter-clockwise from the $X$ axis) of one of the verteces of the polygon: 0 gives a vertex at 12 o'clock, 45 a vertex at $1: 300^{\prime}$ clock, 180 a vertex at 9 o'clock, and so on. The default value depends on the number of sides of the polygon, and gives "conventional" orientations (triangle pointing straight up, square with flat side down...).

If you enter the number of sides of the polygon as a neqative value, the polygon will be drawn "puckered in" at each side, to give a "star"-shaped figure (figure 1 ). So if you enter the number of sides as -5 instead of 5 , the plotting-symbol will be a 5-pointed star.

\section{PLOTTING-SYMBOLS THAT INDICATE ERRORS}

There are 3 plotting symbols that you can use to indicate the errors of the points on the plot: error-box, error-cross, and error-ellipse. If you select one of these symbols, ISOPLOT200 will need to know estimated errors for each of your points. If you select the error-ellipse symbol, ISOPLOT200 will also need the correlation between the $X$ - and $Y$-errors. If you don't know how to estimate the error-correlation, the following relationship may help:

$$
\text { Rho }=\left(E_{x}^{2}+E_{y}^{2}-E_{z}^{2}\right) /\left(2 E_{x} E_{y}\right)
$$

where Rho is the correlation between the $X$ and $Y$ errors, $E_{x}, E_{y}$, and $E_{Z}$ are percent errors in $X, Y$, and $Z$, respectively, and $Z=Y / X$. Error correlations are zero if the error in $X$ doesn' $t$ tend to increase with the error in $Y$. If the error correlations are much greater than about 0.5 , don't use error-box or errorcross symbols, because these symbols will convey a misleading impression as to the actual area of uncertainty on the plot. 
To use an open error-box or error-ellipse, just press the labelled softkey. To get filled error-box or error-ellipse symbols, press the labelled softkey with the SHIFT key.

\section{ENTERING DATA FROM THE KEYBOARD}

As soon as you select a plotting symbol, the CRT will clear and display:

PLOTTING-SYMBOL IS *

\section{2-SIGMA \%-ERRORS}

INPUT DATAFILE SET \#s AS 1st SET, LAST SET-ASTERISK

(e.g. 2.12* or 2.12:15,18*)

[use * $L$ or *R to label data-flle name to ( $L$ )eft or $(R$ )ight of point]

SET\#

87Rb/86Sr Xerr

$875 r / 865 r$ Xerr RHO

\#1: 87Rb/865r, [Kerr,] 875r/86Sr [, Kerr] [,err-corr.] (CONT WHEN DONE)

The axis names and plotting symbols are only examples, of course.

To enter the $X$ - and $Y$-values for your data points, just type in the two numbers, separated by a comma, then press the ENTER key. To include the errors with the points (necessary for error plotting-symbols or for a later Yorkfit), enter 4 values separated by commas, in the format $X, X$-error, $Y, Y$-error. The default error-format is at the 2-sigma level and in percent (but can be changed: see a later section). If no error correlations are entered (an optional 5 th value), they are assumed to be zero. If the errors for all of the points to be entered are the same, don't bother entering them at this point - you'll be asked to enter them as a group later.

When you've entered all of the points that you want plotted with the particular plotting-symbol or pen color that you chose earlier, press CONTINUE. If you're using an error-symbol (error box, error cross, or error ellipsel as the plotting symbol and you didn't enter values for some of the errors, the computer will query:

ENTER X-Yerr, Y-Yerr [,Err.-Corr.] FOR SETS WITH ZERO ERRORS?

Enter the values requested. Note that, again, the error correlation is an optional value, and also that the assigned errors must be nonzero. 
The computer will then display a list of the data-point values that you just entered, and query:

\section{SET TO BE CORRECTED? (CONTINUE IF OK)}

Check the displayed list for errors, and if you made any, enter the set\# (shown to the left of the $X$ - and $Y$-values) of one of the incorrect data points. Enter the correct values when requested, then continue with the editing process until all of the values are correct. Press CONTINUE, and the data points will be plotted.

THE ADD SCREEN

After the data points are plotted this display will appear on the CRT:

PRESS GRAPHICS TO UIEW PLOT, ALPHA TO UIEW THIS SCREEN

PRESS ADD TO INCLUDE THESE POINTS WITH OTHERS FOR A YORKFIT

PRESS DELETE TO DELETE A POINT FROM THE LAST SET

PRESS NEW POINTS TO PLOT A NEW BATCH OF POINTS

PRESS CTRL L TO DRAFT A PHRASE ON THE PLOT

PRESS YORKFIT FOR YORKFIT

PRESS NEW PLOT TO START A NEW PLOT

(press NEW POINTS if you just want to return to the data-entry screen)

At this point, you can choose to plot more points, do a Yorkfit, or abandon the current plot and start a new one. If your plot is on the CRT, you can toggle between the graphics display and the alphanumeric display (the one above) with the GRAPHICS and ALPHA keys to the upper-right of the keyboard. If you press the NEW POINTS softkey, the program will return to the "SELECT PLOTTING-SYMBOL" display, and any additional data points that you enter will be counted as a new batch for regression-line purposes.

But if you want to plot more points (perhaps with a different plotting-symbol or pen color) that are to be pooled with the just-plotted points for a later Yorkfit (or other regression), press the ADD softkey. 
If you don't want to include (for subsequent Yorkfit or other regression) one or more of the points that you just plotted, press the DELETE softkey. The CRT will ask you which point to delete.

\section{LABELLING WORDS OR PHRASES ON THE PLOT}

You can have the program draft any phrase you want anywhere on the plot by pressing the CTRL L key from the "SELECT PLOTTINGSYMBOL" display or the "ADO POINTS" display. The CRT will query:

\section{(PRESS CONTINUE TO ESCAPE)}

CHAR.-HEIGHT? (axis tick-labels were 4) [, ROTATION]

Enter at least one value: the height of the letters in millimeters. You can specify the rotational orientation (counterclockwise from the $X$-axis) of the phrase with an optional second value. So an input of

$$
5,90
$$

specifies a phrase whose characters are about 5 millimeters high and oriented vertically (parallel to the $Y$-axis. If you entered just 5 , the phrase would be oriented horizontally.

After selecting the size and rotation of the phrase, the CRT will instruct you to move the cursor (for a CRT plot; the cursor is a cross-shaped symbol) or the pen (for a plotter plot) with the KNOB to indicate where the lower-right corner of the phrase is to be located. Or you can type in a specific $X-Y$ coordinate by pressing the ENTER $X-Y$ softkey. Once you've specified the location of the phrase, just type it in and enter it with the ENTER key. You can specify superscripts or subscripts with the " and * keys, just as in the axis labels. You can also specify the \pm symbol with the characters $+/-$.

\section{STORING AND RETRIEUING DATA FROM DATA FILES}

You can use the commercially available UISICALC program to construct, edit, and store datafiles that can by read by ISOPLOT200. The UISICALC data flle must conform to a specific format to be compatible with ISOPLOT200, however. This format requires that:

1) the column-width be 9 characters (the VISICALC default); 
2) the column names must appear above any data, and directly overlie a line of repeating equals-signs $(====x====)$;

3) the column names may occupy up to 2 cells above the repeating equals-signs;

4) the first 2 columns ( $A$ and $B$ ) are used for sample names only;

5) no more than 200 rows and 50 columns are allowed;

6) the first row of the spreadsheet can be used as a file title (up to 80 characters) to identify the data file;

7) the file must be stored as a /PF file, not as a /SS file.

8) the file must be stored while the PRINTER WIDTH is defined as 80 .

9) the file name (as stored on the disk) can include only the characters $A$ through $Z$ (caps or lower case) and numbers 0 through 9 .

Because /PF files can't be edited, you should also store the UISICALC file in the normal way - as an /SS file. To make sure that the printer width is 80 , type /PN, enter 7 (the printer select-code), enter 1 (the HPIB address), then enter 80 .

ACCESSING DATA FILES FROM ISOPLOT200

To get data from a data file, press the softkey labelled GET DATAFILE from either the initial display or the "SELECT PLOTTING SYMBOL" display. The CRT will then display: 
Press $k$ for a catalog of the disk in the left-hand drive

Press $\underline{k} 1$ for a catalog of the disk in the right-hand drive

Press $\underline{\text { K2 }}$ or CTRL H for HELP

Press $k 3$ to print out the datafile-data on the printer

Press $k 4$ to load a Visicalc datafile into memory

Press k9 to escape.

(No datafile currently in memory)

DISPLAY ON CRT ONLY

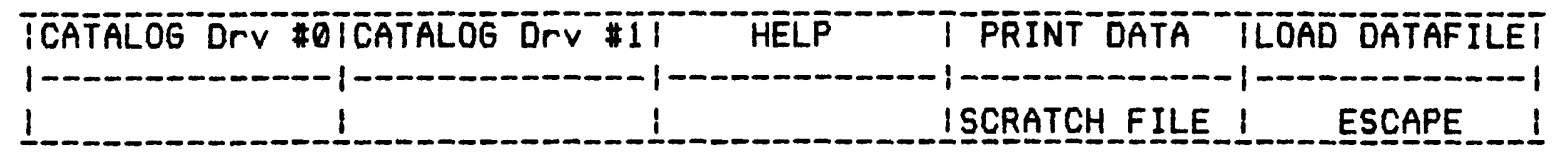

To access data from a data file, if you're sure of its name, press $k 4$. If you're not sure of the name of the file you want, or if you want to see what files are present on a disk, press either $k 0$ or $k 1$. The CRT will then show the names of all of the files on that disk, and indicate those files which are compatible with ISOPLOT200 by highlighting their names. You can then choose one of the compatible files just by entering the number of that file, as shown on the CRT.

Once you've chosen a data file to access, the computer will search for and display a list of all of the column names (headings) for that file. You must then select either 2 columns ( $X$ and $Y$ ), 4 columns ( $X, X$-error, $Y, Y$-error), or 5 columns ( $X, X-$ error, $Y, Y$-error, error-correlation) to get data from.

The computer will then get the data from the disk, and printout the values either on the CRT (default) or the printer (if you pressed $k 3$ on the initial GET-DATAFILE display to specify the printer as the datafile output device).

\section{USING DATA FROM DATA FILES}

To plot data from a data file, you need only refer to the data by "set numbers", where the set number of a data point corresponds to its row in the UISICALC spreadsheet, and is printed out when the data file is accessed by ISOPLOT200. For example, after you've selected a plotting symbol, the CRT display will be: 


\section{PLOTTING SYMBOL IS ERROR-ELLIPSE}

\section{2-SIGMA X-ERRORS}

INPUT DATAFILE SET \#s AS 1 st SET, LAST SET-ASTERISK

(e.g. 2,12* or 2,$12 ; 15,18 *)$

[use *L or *R to label data-file name to ( $L$ )eft or $(R)$ ight of point]

To plot the data for set number 5, enter 5*; to plot the data for sets $5,6,7,8,9$, and 10 , enter 5,10*. To plot data for sets $5,6,7,8$, and $15,16,17$, enter 5,8:15,17*. In other words, indicate continuous sequences of sets with a comma, separate different continuous sequences with a semicolon, and always add an asterisk at the end. It's $O K$ to $m i x$ data file and keyboardentered data, of course.

You can have the computer label each point with the appropriate sample names (from columns $A$ and $B$ in the spreadsheet) by adding an $L$ or an $R$ to the right of the asterisk. An $L$ will result in the names being labelled to the left of the data point, an $R$ to the right of the data point. It's up to you, of course, to beware of any over-writing that this causes.

\section{Using Keyboard-Entered Data as a Temporary Data File:}

If you're entering data-points from the keyboard, and would like to temporarily "file" those data-points for subsequent replotting (perhaps you didn't get everything perfect the first time?), you can do so in the following way. First, press the GET DATAFILE softkey (present in the initial CRT display and the "SELECT PLOTTING-SYMBOL" display). If the GET DATAFILE display indicates that there is a data file currently in memory, press the SCRATCH FILE softkey. ESCAPE from this screen.

With no data file in memory, each data point that you enter is added to a temporary data file, in the sequence that you enter the points. Only the last batch of-data that you enter will be saved, so that the file will consist only of $\underline{N}$ points, where $\underline{N}$ is the highest set-number that you entered. In other words, if you type in the $X-Y$ values for 20 data points, press the NEW POINTS key, then type in another $10 X-Y$ values, the temporary data-file will consists of the second set of 10 points plus the firstentered points 11 through 20 .

To re-plot these points, just treat them as points in a data file with sample numbers 1 through 20. 


\section{YORKFITS OF DATA}

\section{YORKFIT "MODELS"}

A "Yorkfit" refers to a linear regression using the general algorithm developed by Derek York (York, 1969), that weights each point according to both its $X$ - and $Y$-errors and its $X-Y$ error correlation. ISOPLOT200 uses both the original York algorithm and a few modifications, depending on the type of data that you are working with and the amount of scatter that the data shows about a straight line. Each of these modifications, called MODELS by ISOPLOT200, makes different assumptions about the reason for the scatter of the points from a straight line.

The MODEL-1 Yorkfit (York's original algorithm) assumes that the only cause of scatter from a straight line is the errors that you assigned to the points, and weights the points proportional to the inverse square of these errors. A test of this assumption is provided by the PROBABILITY OF FIT value calculated by ISOPLOT200. If this value is reasonably high (more than 0.15 to 0.2 ), then the MODEL-1 assumptions are probably (but not certainly) justified. If this probability is low (less than 0.2 ), however, you may choose another model. The MODEL-1 errors are calculated using the maximum-likelihood algorithm of Titterington and Halliday (1979).

A MODEL-2 Yorkfit assigns equal weights and zero errorcorrelations to each point. This 15 probably seldom valid in terms of any physical model, but at least avoids the mistake of weighting the points according to analytical errors when it is clear that in fact some other cause of scatter is involved.

A MODEL-3 Yorkfit assumes that the scatter is due to a combination of the assigned errors plus a normally-distributed variation (of unknown magnitude) in the Y-values. This model (similar to the Model 3 algorithm of McIntyre and others, 1966) may be realistic in the case of Rb-Sr isochron data for young rocks, where one may expect that the initial $87 / 86$ ratio may have been different for each sample. For this model, ISOPLOT200 will solve for the unknown Y-variation as-well as the best-fit line, so you can judge if the solution is realistic.

A MODEL-4 Yorkfit is used only for U-Pb isotope data on the Concordia diagram. This model assumes that the points scatter due to a combination of the assigned errors plus errors in the $207 / 206$ values that increase linearly from the upper concordiaintercept to the lower concordia-intercept (or vice-versa). This model (see Davis, 1982, for another approach to this problem) may be appropriate for cases where a multi-episodic disturbance is suspected (in which case the closer a point is to the upper intercept the greater its weight), and cases where significant inheritance is suspected (in which case the closer a point is to 
the lower intercept the greater its weight). You may choose whether the lower concordia-intercept or the upper concordiaintercept is to be the age of interest.

The program will always attempt a MODEL-1 fit first. In the Yorkfit printout, the A PRIORI errors are the errors in the slope and intercept calculated only from your assigned errors for each point. In other words, no matter how much the points scatter from a straight line, the APRIORI errors will remain the same. The INCLUDING SCATTER errors, however, are calculated from the actual scatter of the points from the line, so the less the scatter, the lower the INCLUDING SCATTER errors.

How the $95 \%$-confidence limit errors are calculated depends on the probabilility that the assigned errors for the points can account for the observed scatter. If this probabilility is greater than 0.15 , the $95 \%$-confidence limit errors are simply 1.96 times the A PRIORI errors ( 1.96 is the Student's-t value for infinite degrees of freedom - the reason for this is discussed by Brooks and others, 1972). If the probability is less than 0.15 , the $95 \%$-confidence limit errors are $t$ times the INCLUDING SCATTER errors, where $\underline{t}$ is the Student's- $\underline{t}$ value for $N-2$ degrees of freedom ( $N$ = number of points). If you choose the MODEL-1 Yorkfit when the probability is low, you are in effect assuming that the true analytical errors are greater than your original estimate by some unknown factor, but that the relative $X$ - and $Y$ errors, and the error-correlations, are still valid.

The MOOEL-3 Yorkfit is offered as an option if the program recognizes, from the axis names, that you are plotting isochron data for Rb-Sr, Sm-Nd, U-Pb $(238 / 204-206 / 204$ or $235 / 204$ $207 / 204)$, or Th-Pb $(232 / 204-208 / 204)$. The $95 \%$-confidence limit errors are calculated from $t$ times the INCLUDING SCATTER errors, where the $t$ value is for $\mathrm{N}-2$ degrees of freedom. The MODEL-2 95\%-confidence limit errors are also calculated in this way.

The MODEL-4 Yorkfit is offered only for Concordia-diagram data, and only for data-sets with at least 6 points. The 95\%confidence limit errors are calculated by multiplying the INCLUDING SCATTER errors by the $t$ value for $N^{\prime}-2$ degrees of freedom, where $N^{\prime}$ is a number that is in general less than the actual number of points. This approach seems necessary because of the way in which the MODEL-4 algorithm weights the points the more concordant the point, the greater its weight. In the extreme case, with 2 nearly concordant points plus any number of much more discordant points, the algorithm in effect pays attention only to the 2 most-concordant points and ignores the rest. Obviously in this case, the true degrees of freedom are much less that the apparent degrees of freedom. To take this into account, $N^{*}$ is calculated by assigning each of the 2 most-concordant points a "point value" of exactly 1 , and all of the rest of the 
points a fractional "point value" based on their weight relative to the second-most concordant point.

The effect of this procedure (which is intuitive rather than mathematical) is to drasticaly reduce the degrees of freedom assigned to many data sets, especially if 1 or 2 of the points in the data set are much more concordant than the rest. So the data sets which are most appropriate for the MODEL-4 approach are those with a large number of points that are relatively evenly spaced along a linear trend. Data sets with few points will tend to have MODEL-4 errors that are very large, due to the large (perhaps several tens or hundreds) Student's-t multiplier invoked. You can tell the value of $N^{\prime}$ used by the algorithm from the printout, which refers to the NUMBER OF EQUIUALENT "POINTS".

\section{OBTAINING ISOCHRON AGES FROM YORKFIT LINES}

If your plot is an isochron plot (including ${ }^{206} \mathrm{~Pb} /{ }^{204} \mathrm{~Pb}-$ $207 \mathrm{~Pb} /{ }^{204} \mathrm{~Pb}$ and $\left.{ }^{204} \mathrm{~Pb} / 206 \mathrm{~Pb}-{ }^{207} \mathrm{~Pb} / 206 \mathrm{~Pb}\right)$, the computer will automatically calculate an isochron age and error from the Yorkfit results. The decay constants used are those recommended by the I.U.G.S. Subcommission on Geochronology (Steiger and Jager, 1977).

\section{RE-USING YORKFIT POINTS and PLOTTING ERROR-ENUELOPES}

After ISOPLOT200 has completed a Yorkfit, the softkeys will be defined as:

IERROR_ENUELOPE!
IEREI

If you press CONTINUE, you will return to the "SELECT PLOTTING-SYMBOL" display, and any any new data-points that you enter will count as a new "set" of points for subsequent Yorkfits or other regressions. If you want to either add to or delete from the set of points that was just Yorkfit, press the appropriate softkey. If you want to plot the $95 \%$-confidence limit error-envelope about the Yorkfit line, press the ERROR ENUELOPE softkey. You can request that the error-envelope be drawn, incidentally, by pressing CTRL $E$ from most of the CRT displays that have to do with data entry. 
SELECTING A DASHED OR SOLID YORKFIT LINE

The Yorkfit line will be drawn as a dashed pattern (figure 2), unless you specify a solid line by pressing the SOLID LINE softkey from the "SELECT PLOTTING-SYMBOL" screen. The dashed pattern will change with each successive Yorkfit that you request for a given plot.

\section{THE OPTIONS SCREEN}

The OPTIONS screen (available from the "SELECT PLOTTING SYMBOL" screen) allows you to fit a curve to data, change the error-input format, store a CRT plot on a disk, or recall a CRT plot from a disk. The softkeys are labelled as follows:

\section{MISCELLANEOUS OPTIONS}

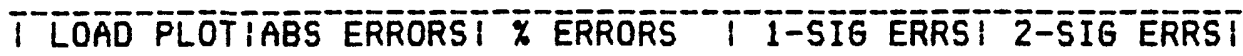

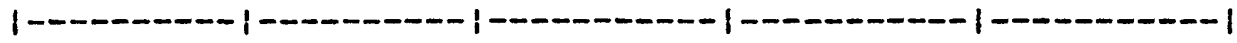
ISTORE_PLOT!_EXPO_FIT_LLOG FIT_ I POLYFIT__ ESCAPE_!

The EXPO FIT, LOG FIT, and POLYFIT options will fit a leastsquares curve to the last set of plotted data, of the form $Y=A * E^{(B * X)}$ [EXPOFIT], $Y=A * \operatorname{LOG}(B * X)$ [LOG FIT], or a polynomial of order 1 to 4 .

The \% ERRORS, 1-SIG ERRORS, and 2-SIG ERRORS options allow you to change the error-input format from the default one of 2sigma(\%) to the 1-sigma level and/or absolute rather than percent errors.

The LOAD PLOT and STORE PLOT options allow you to store the image of a CRT plot on a disk, or to retrieve that image from a disk. This way, you can store a plot with data-points for later retrieval and possible additional plotting. 


\section{CREATING CONCOROIA PLOTS}

If your plot is a concordia plot $\left({ }^{206} \mathrm{~Pb} /{ }^{238} \mathrm{U}-{ }^{207} \mathrm{~Pb} /{ }^{235} \mathrm{U}\right)$, you'll find a few differences from the normal $X-Y$ plotting rou$t$ ine. First, if the program is in the ISOCHRON or $X-Y$ PLOTTER mode, you must return to the starting screen (NEW PLOT softkey from the "SELECT PLOTTING-SYMBOL" screen). When the initial ISOPLOT200 screen is present, press CTRL C to indicate a concordia plot. Set up the plot in the usual way (CRT or plotter, pennumber, et cetera), then press the START PLOT softkey. The program will then ask you to define the limits of the plot in terms of anes, rather than $X-Y$ values. The display will be:

ENTER AGE LIMITS AS: [MIN. AGE,] MAX. AGE

\section{(or press CONTINUE to define in terms of $X$ and $Y$ limits)}

If you enter 1 value only, the minimum age to be shown on the concordia plot is assumed to be 0 . If you enter 2 values, the plot will show ages from about the first value to about the second value (generally, a slightly larger age-range so that the axis-ticks will start and end on reasonable numbers).

If you want to define the plot limits in terms of the actual $X$ - and Y-values, though, just press CONTINUE in response the the query, and you can do so.

The concordia curve can be drawn with 2 kinds of ticks and tick-labels: ticks as small circles labelled horizontally to the left of the $t i c k$, and ticks as short dashes normal to the concordia curve, with labels above and colinear with the ticks. You can select which style you want from the initial screen, where softkey $k 3$ will be labelled either DASH CTICK or CIRCLE CTICK

An additional feature of both the "SELECT PLOTTING-SYMBOL" and "ADD POINTS" screens for concordia plots is the option to force the next Yorkfit through a given age on the concordia curve. The added prompt is:

press CTRL $F$ to force the Yorkfit line through a point on concordia

After pressing CTRL $F$, the CRT will ask you the age that you wish to force the Yorkfit line through.

Also, after any Yorkfit, the program will calculate the concordia intercepts of the Yorkfit line and its associated uncertainties, using the algorithm of Ludwig (1980). These intercepts will be labelled on the lower-left corner of the plot (optional if a plotter plot), with the labels for each successive solution for a given plot stacked on the previous one. 


\section{OBTAINING GROWTH CURUES FOR PD-ISOTOPE PLOTS}

If your plot is either a ${ }^{207} \mathrm{~Pb} /{ }^{204} \mathrm{~Pb}-{ }^{206} \mathrm{~Pb} / 204 \mathrm{~Pb}$ or a ${ }^{208} \mathrm{~Pb} /{ }^{204} \mathrm{~Pb}-{ }^{206} \mathrm{~Pb} /{ }^{204} \mathrm{~Pb}$ plot, after drawing the plotbox and labels the computer will ask:

PRESS KO TO GET A STACEY-KRAMERS GROWTH-CURUE,

PRESS K\ TO GET SOME OTHER SINGLE-STAGE GROWTH-CURUE.

PRESS Kg TO DECLINE ANY GROWTH-CURUE.

If you press k0, the computer will draw a single-stage $\mathrm{Pb}-$ isotope growth-curve on the plot using the constants suggested by Stacey and Kramers (1975). If you press $k 1$, the computer will ask you to define your own single-stage growth curve, in terms of the starting age, the initial isotope ratios, the Mu of the source, and (for 208/204 - 206/204 plots) the Th/U of the source.

\section{DUMPING A CRT PLOT TO THE PLOTTER OR PRINTER}

You can obtain a hard copy of any CRT plot by requesting that the CRT plot be "dumped" to either the printer or the plotter. Press CTRL P from the "SELECT PLOTTING SYMBOL" screen to dump the plot to the printer. This will only work, incidentally, if the printer is one of the types that can accept direct graphics-dumps, such as the HP-2225 ThinkJet printer. The HP$82905 B$ dot-matrix printer is not such a printer. To dump the CRT plot to the plotter, press CTRL D from the "SELECT PLOTTING SYMBOL" screen. The plot will be transferred to the plotter using plot-size 8 and pen-number 1. 


\section{REFERENCES}

Brooks, C., Hart, S.R., and Wendt, I., 1972, Realistic use of two-error regression treatments as applied to rubidiumstrontium data: Rev. Geophys. Space Phys., v. 10, p. 551577 .

Davis, D.W., 1982, Optimum linear regression and error estimation applied to U-Pb data: Canadian Journal of Earth Sciences, $v$. 198 , p. 2141-2149.

Ludwig, K.R., 1979a, A program in Hewlett-Packard BASIC for X-Y plotting and line-fitting of isotopic and other data: U.S. Geological Survey Open-File Report 79-1641; 33 p.

-...-.-... 1979b, A program in Hewlett-Packard BASIC for plotting and processing U-Pb isotope data on "concordia" diagrams: U.S. Geological Survey Open-File Report 79-1692; 32 p.

-............ 1980, Calculation of uncertainties of U-Pb isotope data: Earth Planetary Science Letters, v. 46, p. 212-220.

-.-.-.-. 1982, Programs for filing and $X-Y$ plotting of isotopic and other data using an HP-9830 computer and HP-9832 plotter: U.S. Geological Survey Open-File Report 82-385; 36 p.

- 1982, Programs for filing and plotting U-Pb isotope data for concordia diagrams, using an HP-9830 computer and HP-9862 plotter: U. S. Geological Survey Open-File Report $82-386 ; 22$ p.

- 1983, Plotting and Regression programs for isotope geochemists, for use with HP-86/87 microcomputers: U.S. Geological Survey Open-File Reportfile Report 83-849; 89 p.

Processing Raw Pb-U-Th Isotope Data: U.S. Geological Survey
Prom Open-File Report.

MeIntyre, G.A., Brooks, Christopher, Compston, William, and Turek, Andrew, 1966, The statistical assessment of Rb-Sr isochrons: Journal of Geophysical Research, v. 71, p. 54595468 .

Stacey, J.S., and Kramers, J.D., 1975, Approximation of terrestrial lead isotope evolution by a two-stage model: Earth Planetary Science Letters, v. 26, p. 207-221. 
Steiger, R.H., and Jager, Emilie, 1977, Subcommission Geochronology: Convention on the use of decay constants in geo- and cosmochronology: Earth Planetary Science Letters, v. 36, p. 359-362.

Titterington, D.M., and Halliday, A.N., 1979, On the fitting of parallel isochrons and the method of maximum likelihood: Chemical Geology, v. 26, p. 183-195.

York, Derek, 1969, Least-squares fitting of a straight line with correlated errors: Earth Planetary Science Letters, $v$. 5, $p$. $320-324$. 


\section{FIGURE CAPIIONS}

Figure 1: $X-Y$ plot showing various plotting symbols. Top left row - alphanumeric (keyboard) symbols; top right row polygon symbols; lower - error symbols. Curve is a polynomial fit through the dots. Plot size is 6 .

Figure 2: Concordia plot with Yorkfit line through error-ellipse symbols. Oblique-dash concordia ticks.

Figure 3: Pb-isotope plots created with the "nonstandard" plotsize option and the STACK option for the upper plot. Growth curves are standard Stacey-Kramers (Stacey and Kramer5, 1975). 


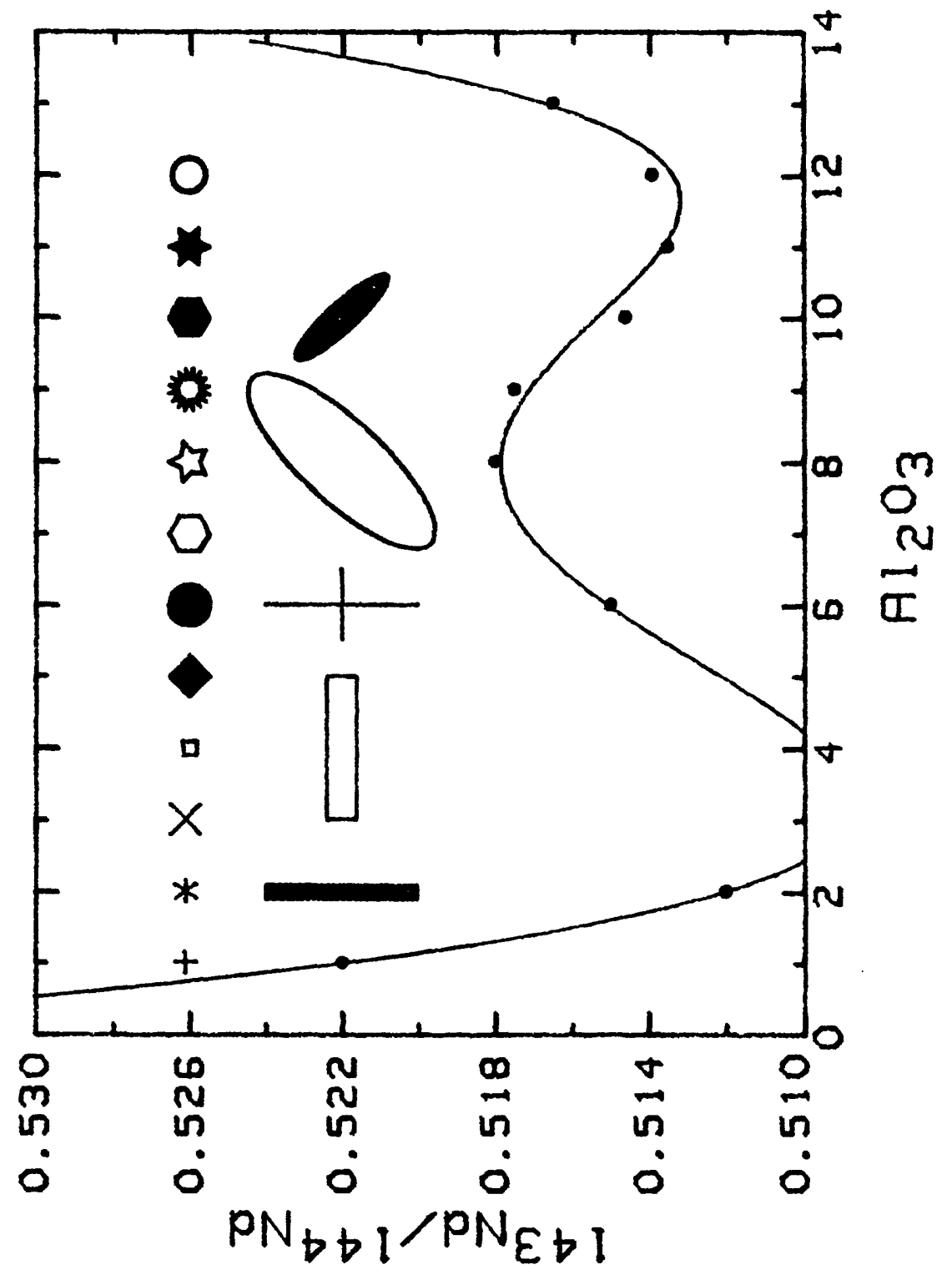

Figure 1 


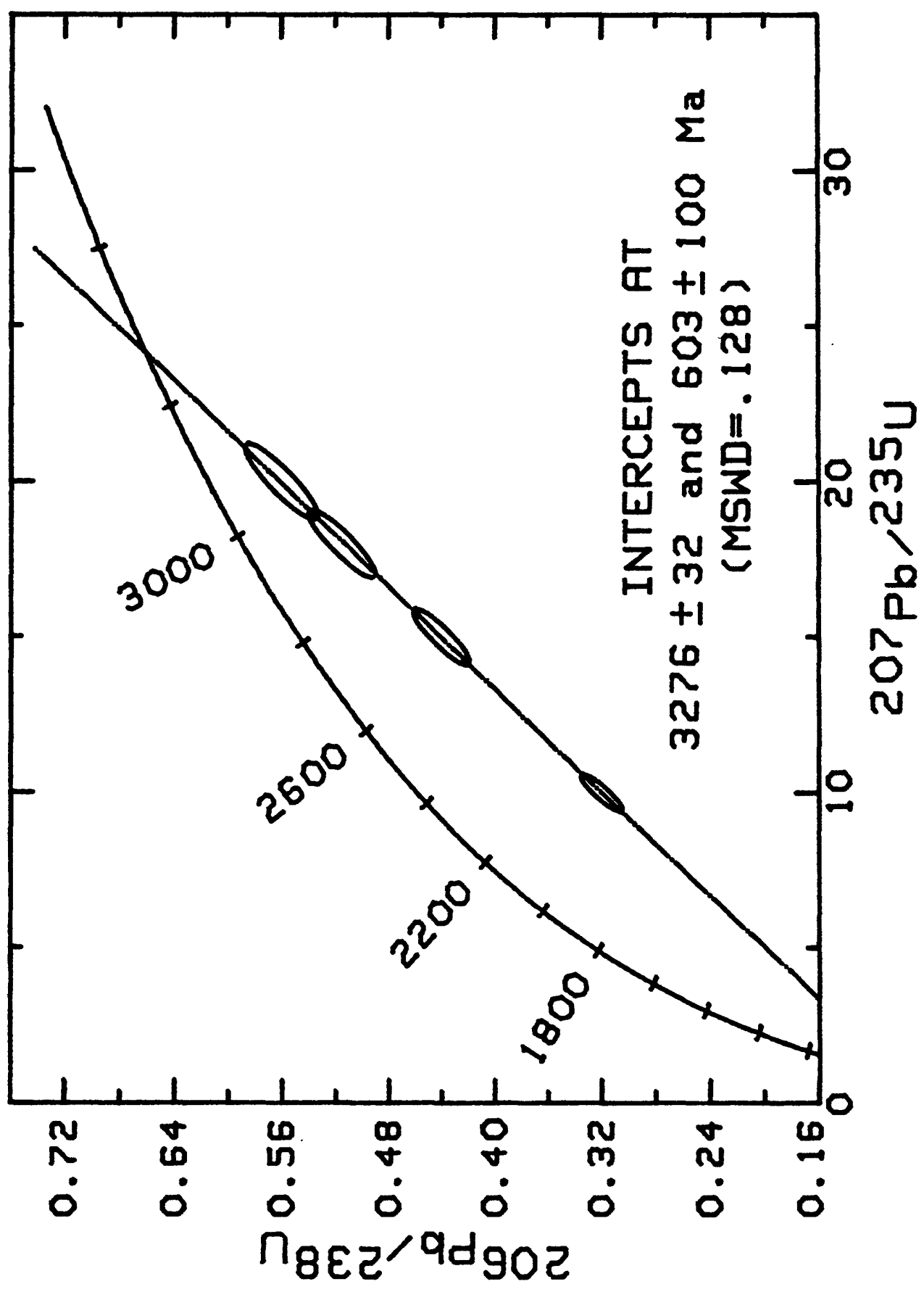

Figure 2 


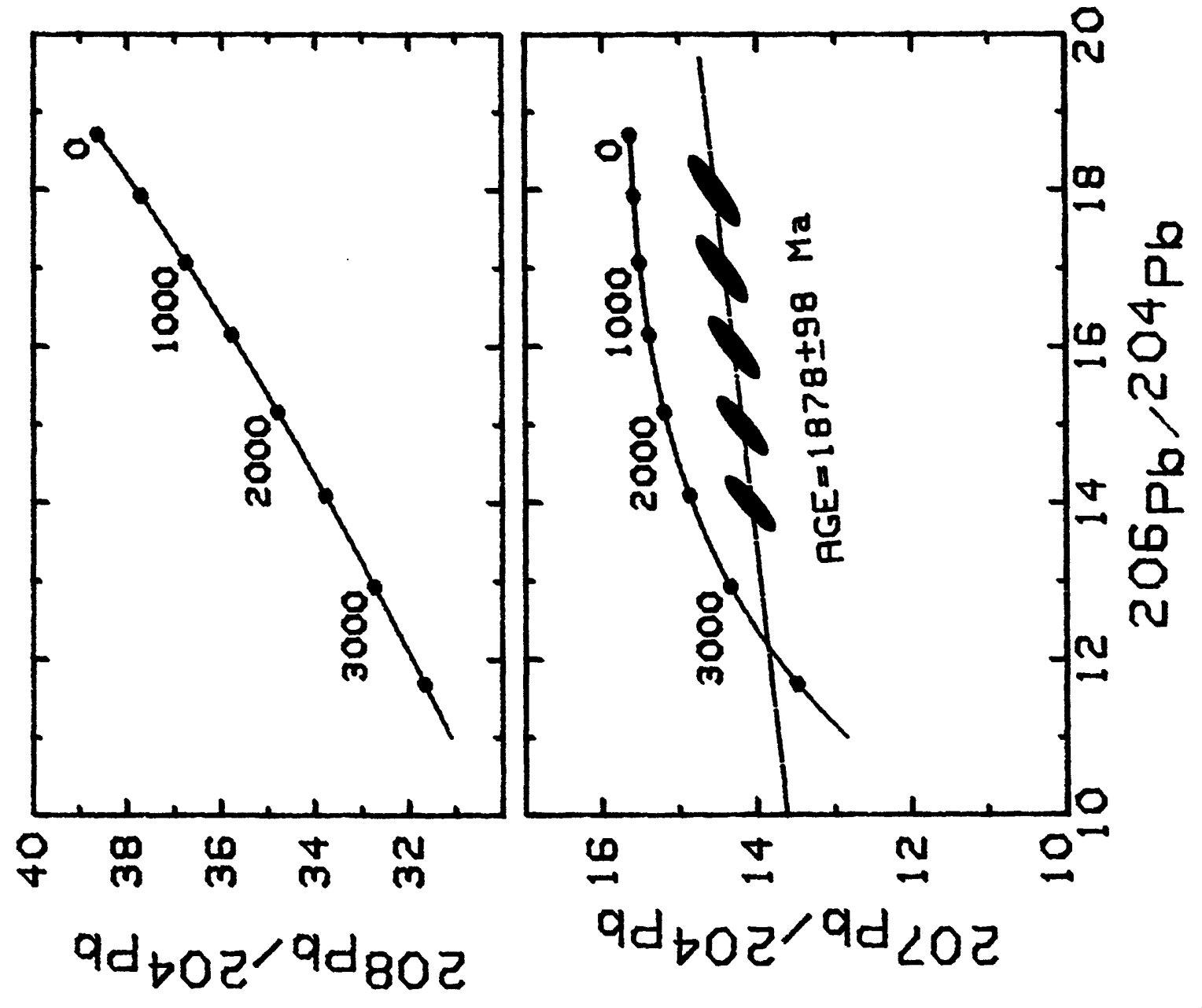

Figure 3 


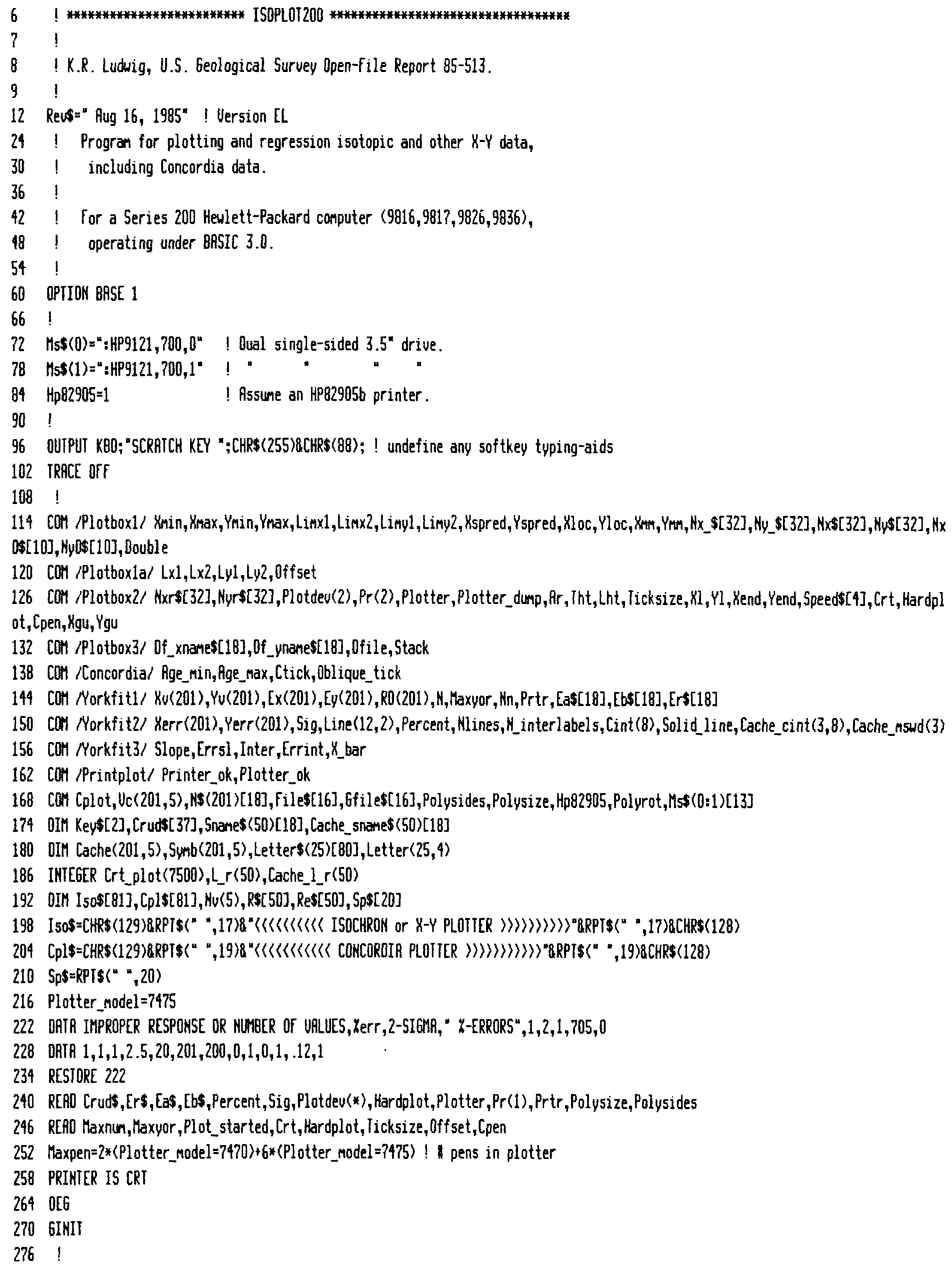


282 Redraw:0月Th $0,0,0,0,0,0,0,0,0,1,1,0,9$, US

288 RESTORE Redraw

294 Reno Stack, Sizetype,Plotter_dump, Solid__line,Nlines, N_interlabels, Oblique_tick, Nphrases, Bypass, Pr (2), Prtr, __ct, Penspeed, Speed\$

300 OFF DELAY

306 DEG

312 Clear

318 If Hardplot IHEN PEN 0 ! Put plotter-pen away if out

324 !

330 Begin:Clear ! Set up initial screen 8 aptions

336 Alpha

342 OFF KEY

348 Neuplot=1

354 Slope $=0$

360 PRINIER IS CRI

366 !

372 PRINT IABXY(1,1);"Rev. ";Reus;1AB(51);"K.R. Luduig, U.S. Geol. Survey"

378 If Iicksize $>1$ IHEN DISP "RELATIUE LABEL-SIZE =";Ticksize

384 If Hardplot IHEN

390 PRINT IAGXY(1,4); "PLOTTER PLOT"

396 If Rotate_90 THEN PRINT TRBYY $(1,7)$; "ROTATED PLOT"

402 If HOT Rotate_90 THEN PRINT TABXY $(1,7) ; "$

408 If Stack IHEN PRINT IABXY(1,8); "SUPRESSED X-AXIS \& 8 -IICK LABELS"

414 If HOT Stack THEN PRIHT TABXY $(1,8) ; "$

420 ON KEY 5 LABEL "ROJ. LABELSIZE" GOTO Labelsize

426 ON KEY 1 LABEL " CRT PLOT" GOTO Crtplot

432 ON KEY 2 LABEL " CHOOSE PEN" GOTO Choose_pen

438 OH KEY 7 LABEL " PEE SPEED" GOTO Penspeed

444 If NOT Cplot AND NOT Stack THEN ON KEY 3 LABEL " STRCKED PLOT" GOTO Stack

450 If NOT Cplot AND Stack THEN ON KEY 3 LABEL "UHSTRCKED PLOT" GOTO Stack

456 If Rotate_90 THEN ON KEY 6 LABEL "UNROTATED PLOT" GOTO Rotate_plot

462 If NOT Rotate_90 IHEN ON KEY 6 LABEL " ROTATED PLOT" GOTO Rotate_plot

468 ELSE

474 PRINT TABXYY(1,4); "CRT PLOT"

480 ON KEY 1 LABEL " PLOTIER PLOT" GOTO Hardplot

486 OH KEY 3 LABEL " CALL Clunk

492 END IF

498 If Prtr=1 THEN

504 PRINT TABRY (67,4); "CRT OUIPUI"

510 ON KEY O LABEL "PRINTER OUTPUT" GOTO Hardcopy

516 ELSE

522 PRINT IABXYY(67,4); "PRIHTER OUTPUT"

528 ON KEY O LABEL " CRT OUTPUI" 68TO Display

534 ENO IF

540 If Cplot THEN

546 PRINT TABXYY $(1,2) ;$ Cp 15

552 PRINI TheXY(1,17);FNCenters ("Press "8FFUns ("CIRL I")8" for isochron or other $X-Y$ plot")

558 If Oblique_tick IHEN

564 PRINT TABXY 25,4 ); "OBLIQUE-OASH CONCOROIA IICKS"

570 ON KEY 3 LABEL " CIRCLE CIICK" GOTO Mornaltick

576 ELSE

582 PRIHT TABXY 25,4$) ;$ "HORIZ. -CIRCLE COMCORDIA IICKS"

588 ON KEY 3 LABEL " BASH CIICK" GOTO Obl iquetick

594 END IF

600 ELSE

606 PRIAT TABXYY(1,2);Isos

612 PRIMT TABXY(1,17); FHCenter\$("Press "BFHUns("CIRL C")8" for concordia plot")

618 END IF

624 !

630 OH KEY 4 LABEL " HELP" GOTO Call_helpl

636 ON KEY 9 LABEL " STRRT" GOTO 60 
642 ON KEY 8 LABEL " GET DRTAFILE" GOTO Get_datafile

648 !

654 React1:ON KBO 60TO Keys1

6606010660

$666 !$

672 Keysl:K=FNGet_key I trap non-sof tkey press

678 Off KBD

684 !

690 SELECT K

696 CASE 8

! CTRL-H key pressed

702 Call_help1:Help1 (Cplot)

708 G0T0 Begin

714 CASE $-6 ?$

7206070 6o

726 CASE 3,9

732 Colot $=(K=3)$

738 BEEP 440,.1

744 GOTO Begin

750 CASE $-185,-195$

756 If Hardplot IHEN

762 Bypass=1

$768 \quad 607060$

774 ELSE

780 Clunk

386 END If

792 CASE ELSE

798 Clunk

804 END SELECT

8106070 React1

$816 !$

822 60: I request operator to define plotbox \& then draw plotbox

828 OFF KEY

834 OfF KBD

840 If Crt THEN Ticksize $=1$

846 Double $=$ Hardplot*(Penspeed $=6$ OR Ticksize $\rangle=1.5) *$ (NOT Bypass) ! draw sone parts of plot twice if slow penspeed or large label-siz

e

852 CALL Plotbox (Cruds, CPlot, Rotate_90,Bypa55,Plot_started, Escape)

858 If Escape THeN Begin

864 If Cplot AND NOT Bypass THEN CALL Concordia ! draw \& label concordia

870 If NOT Cplot THEN CALL Pbgrowth

876 If Plotter_dump THEN RETURN

882 PEN 0

8886070 Startplot

$894 !$

900 Stack:Stack=NOT (Stack〉 ! suppress $\mathrm{X}$-axis label \& X-axis tick-labels?

906 BEEP 440,.1

9126070 8egin

$918 !$

924 Hardcopy:8EEP 440,.1 ! Use printer as printing device

930 Printer_ok=1

936 ON TIMEOUI ?,.1 CALL Printer_timeout

942 PRINTER IS 701

948 PRINT

954 If Printer_ok THEN

960 OFF IIMEOUI

$966 \operatorname{Pr}(2)=701$

972 Prtr $=2$

978 ENO If

984 PRINTER IS CRT

990 60TO Begin 
1008 Rotate_plot:Rotate_90=NOT (Rotate_90) I Rotated plot?

1014 BE[P 440,.1

10206010 Begin

1026 !

1032 Display: BEEP 440, 1 ! Use CRT as printing device

$1038 \operatorname{Pr}(2)=C R T$

$1044 \operatorname{Prtr}=1$

1050 PRINTER IS CRT

1056 GOTO Begin

1062 !

1068 Hardplot:BEEP 440,.1

! Use HP-7475A as plotter

1074 Plotter=Plotdeu $\{2\rangle$

1080 Plotter_ok=1

1086 ON IIMEOUT ?,.1 CALL Plotter_timeout

1092 PLOIIER IS Plotter, "HPGL"

1098 If Plotter_ok IHEN

1104 OFf IIMEOUT

1110 Hardplot $=1$

$1116 \quad \mathrm{Crt}=0$

1122 PEN 0

1128 ENO If

1134 G0TO Begin

$1140 !$

1146 Crtplot:DISP FHHS("CRT GRPPHICS")BSp\$ ! Use CRT as plotter

1152 BEEP $440, .1$

1158 Plotter $=1$

1164 Hardplot=0

$1170 \mathrm{Crt}=1$

1176 60T0 Begin

$1182 !$

1188 Normaltick:0blique_tick=0 ! for a concordia plot, use an " 0 " for the concordia tick \& label the ticks horizontally

1194 BEEP 440, .1

12006070 Begin

1206 !

1212 obliquetick:Oblique_tick=1 ! for a concordia plot, use a short dash for a tick, perpendicular to the concordia curve, labelled parrallel to the dash

1218 BEEP 440,.1

1224 60TO Begin

$1230 !$

1236 Labelsize:Ticksize=1 ! adjust the relative size of the axis labels, tick labels, alphanumeric plotting symbols

1242 OFF KBD

1248 OFF KEY

1254 Clear

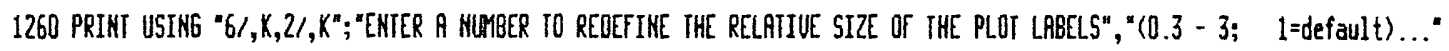

1266 LOOP

1272 INPUT Ticksize

1278 EXII If licksize $\rangle=.3$ AND licksize $\langle=3$

1284 Bad_input("ILLEGHL_SIZE")

1290 ENO LOOP

12966010 Begin

1302 !

1308 Get_datafile:GOSUB Datafile ! get data from a datafile

13146010 Begin

1320 6_datafile: G0SUB Datafile ! ditto, called after plotbox drawn

1326 G0I0 More_points

1332 !

1338 Penspeed:605UB Pen_speed

$134460 T 0$ Reactl 
$1350 !$

1356 Pen_speed: I Define pen-speed for plotter

1362 OISP "PRESS KEY (D-9) TO INDICATE THE PLOTTER PEN-SPEED..."BSpS

1368 REPEAT

1374 ON KBD 60101392

$1380 \quad 60701380$

1386 !

1392 Penspeed $=$ FNGet_key-48

1398 OFF KBD

1404 UNIIL Penspeed $\rangle=0$ AkO Penspeed $=9$

1410 !

1416 If Penspeed $=9$ THEN Speed\$="US" ! fast + nax. acceleration

1422 If Penspeed $\langle 9$ THEN Speed\$="US"8URLS(INT (3B.1*(Penspeed+1)/10))

1428 BEEP 440,1

1434 DISP FNHS("PEN-SPEED "8URLS(Penspeed))

1440 REIURN

1446 !

1452 Kretrn:0Fr KBD

1458 RETURN

1464 !

1470 Startplot: $N_{n}=0$

1476 LDIR 0

1482 If Cplot $=0$ THEN Lines $=$ "ISOCHRON"

1488 If Cplot THEN Line $\$=$ "CHORD"

1494 PRINTER IS CRT

1500 !

1506 Plotdata: OFF KEY

1512 ALPHA OH

1518 DคFT $0,1,1,0,0$

1524 RESTORE 1518

1530 REAO H,_Ii,KK, Kane_label, forced_inter

1536 !

$1542 ! N=$ number of data-points last input

$1548 ! \mathrm{kk}=$ number of first data-point in current set

1554 ! Ii = counter for data-point in current set

$1560 ! \mathrm{N}_{n}=$ number of points in current set

1566 !

1572 More_points:Clear

1578 Mpts:OFF KBD

1584 off $\mathrm{KEY}$

1590 Alpha

1596 PRINTER IS CRT

1602 OK DELLY 3600 GOTO Redraw ! If no user action within an hour, return to

1608

! starting display.

1614 If Sonepoints THEN GRAPHICS OFF

1620 !

1626 ! Set up the plot-points options screen

1632 PRINT TABXY(1,6); FNCenters (FHHS ("SELECT A PLOTIING-SMMBLL TO PLOT OATA"))

1638 If Crt IHEN

1644 PRINT TABaY(20,12); "press "QFNHS ("CTRL O") $)$ " to dump plot to plotter"

1650 PRINT TABXY(20,14);"press "BFWHS ("CIRL P")Q" to dump plot to printer"

1656 END If

1662 PRINT TABXY(20,10);"press "BFHAS ("CTRL L")8" to draft a phrase on the plot"

1668 If Cplot AND Nn THEN PRINT TABXY(20,10); "press "BF NHS ("CTRL-F" $)$ " to force Yorkfit through point on concordia"

1674 PRINT TABXY(20,16); "press "8FFH(S ("CTRL H")8" for help"

$16 B 0$ PRINT TABXY $(20,18) ;$ "press any key for keyboard plotting-symbol"

1686 !

1692 If Hardplot THEN

1698 PEN 0

1704 MOUE Xmin, Ymax 
1710 ENO IF

1716 Keyboard $=0$

1722 Newplot $=0$

1728 !

1734 ! Set up the softkey branches

1740 Reenter:PRINIER IS CRI

1746 If NOT CPlot THEN

1752 ON KEY O LABEL " ERROR BOX" GOTO Debox

1758 ON KEY 10 GOTO Sebox

1764 ON KEY 2 LABEL " ERROR CROSS" 60TO Errcross

1770 END If

1776 ON KEY 1 LABEL "ERROR ELLIPSE" GOTO Deell

1782 ON KEY 11 GOTO Seell

1788 ON KEY 3 LABEL " POLYGOH" GOTO Open_poly

1794 ON KEY 13 60T0 Solid_poly

1800 ON KEY 4 LABEL " OPIIONS" $60 T 0$ Options

1806 If Hardplot THEN ON KEY 6 LABEL " CHOOSE PEN" GOTO Choose_pen

1812 If Crt THEN ON KEY 6 LABEEl " ERRSE PEN?" 60SUB Erase_pen

1818 ON KEY 8 LABEL " GET DATRFILE" GOTO 6_datafile

1824 If Nn>1 THEN ON KEY 7 LABEL " YORKFIT" GOTO Call_yorkfit

1830 ON KEY 9 LABEL " MEL PLOT" GOTO Redraw

1836 ! If Crt THEN ON KEY 5 LABEL * PRINT PLOT" GOTO Print_plot

1842 If Hardplot AKD Solid_line THEN ON KEY 5 LABEL " OASH "Blines GOTO Solid_line

1848 If Hardplot AND NOT Solid_line THEN ON KEY 5 LABEL "SOLID "QLine\$ 60TO Solid_line

1854 !

1860 React2:ON KBO G0TO Kybrd

186660101866

1872 !

1878 ! Select pentll for hard-plotter

1884 Choose_pen:DISP FNHS("PRESS A NUHBER-KEY (1 - "BuALLS(Maxpen)\&") TO SELECI a PEN FOR PLOTING...")

1890 REPEAT

1896 ON XBD $60 T 01908$

190260701902

1908 K=FNGet_key

1914 Off KBO

$1920 \quad$ Cpen $=k-48$

1926 uwIIL Cpen $\rangle=1$ AND Cpen<位xpen

1932 BEEP 440,.1

1938 DISP FNHS ("PENI" "BuALL(Cpen))

1944 on 1+Newplot G0TO React2, React1

1950 !

1956 Erase_pen: ! Use an "erasing pen "(CRT-plot only)

1962 If Cpen<O THEN

1968 Cpen $=1$

1974 DISP FNHA (" UHIIE PEN ") 8 SpS

1980 ELSE

1986 Cpen $=-1$

1992 DISP FNHS(" ERASING PEN ")RSPS

1998 END If

2004 BEEP 440,1

2010 RETURN

2016 !

2022 Solid_line:Solid_line=HOT (Solid_line) ! use a solid, rather than a dashed line for Yorkfit lines

2028 off KEY

2034 OFF KBD

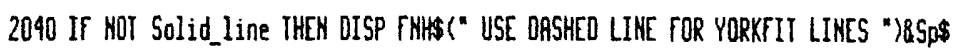

2046 If Solid_line THEN DISP FNHS(" USE SOLID LIHE FOR YORKFIT LINES "885p\$

2052 BEEP 440,.1

$205860 T 0$ Reenter

2064 ! 
2070 Debox:Symbol $=1$

2076 Clear

2082 PRINI FNHS(" OPEN ERROR-BOX SMMBOL ")

20886010 Input_data

2094 !

2100 Sebox: Symbol =-1

2106 Clear

2112 PRINT FHHS(" SOLID ERROR-BOX SYMBOL ")

21186010 Input_data

2124 !

2130 Deell : Symbol $=2$

2136 Clear

2142 PRINT FYHSL" OPEN ERROR-ELLIPSE SMMBOL ")

21486010 Input_data

2154 !

2160 Seell : Symbol $=-2$

2166 Clear

2172 PRIMT FHHS(" SOLID ERROR-ELLIPSE SMMBOL ")

21786010 Input_data

2184 !

2190 Errcross:Symbol $=4$

2196 Clear

2202 PRIHI FHHS(" ERROR-CROSS SMMBOL ")

22086010 Input_data

2214 !

2220 Dpen_poly:Solid $=0$

2226 G0T0 Poly

2232 Solid_poly:Solid=1

22386010 Poly

2244 !

2250 Poly: Symbol=3 I define a polygon as the plotting symbol

2256 Clear

2262 OFF KEY

2268 GRAPHICS OFF

2274 If Solid THEX PRIMT TABXY(1,1); CHPS (129)8" SOLID";

2280 If NOT Solid THEN PRINT IABXY(1,1);CHRS $(129) 8^{\text {" OPEN"; }}$

2286 PRINT " POLYGON SYMBOL "BCHRS (128)

2292 PRINI TAGXY(1,14); "POLYGOH-SMMOLL: ENTER USIDES (- for star), [, SIZE (m) [,ROTAHIOK (0-360)]]"

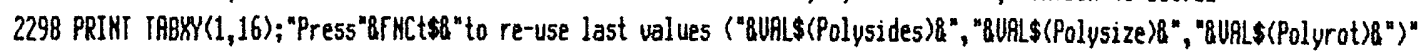

2304 P_kbd:OW KBO $60 T 02328$

2310 OK KEY 9 LABEL " ESCAPE" GOTO More_points

2316 GOTO 2316

2322 !

$2328 \mathrm{~K}=$ FNGet_key

2334 off KBD

2340 OFF KEY

2346 SELECT $K$

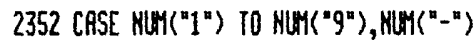

2358 OUTPUT KBD;CHRS(K);

2364 PRINT TABAY $(1,16)$;RPTS(" ",80)

2370 DISP FNASt\$;

2376 Retrieve (Rs, Hu(*), Hinputs, 1)

2382 If $R S=* *$ " IHEN More_points

2388 If Ninputs THEX

2394 Polysides=Hu(1)

2400 Polysize $=$ Hu(2)*(Ninputs)1) $+3 *$ (Ninputs(2)

2406 If Polysize) 40 THEN

2412 Bad_input("A POLYGON of "BuALS(Polysize)\&" m IS TOO LARGE!")

2418 GoTO Poly

2424 END IF 
2430 Polyrot $=H u(3) *($ HInputs $) 2) *\left(90 *\left(P_{0}\right.\right.$ lysides/2=iNT(Polysides/2))*(180/Polysides-90))*(Ninputs(3)

2436 If Hinputs<3 AND Polysides 110 THEN Polyrot=0

2442 END IF

2448 CASE -6 ? I COHTINUE key: do nothing

2454 CASE ELSE ! invalid

$24605010 P_{-}$kbd

2466 END SELECT

2472 !

2478 PRINI TABXY(1,14);RPTS(" ",3*80)

24846070 Input_data

$2490 \quad$ !

2496 Kybrd:Keycode=FMGet_key ! react frof a non-softkey press

2502 off $\mathrm{KBO}$

2508 SELECT Keycode

2514 CASE 8

! CIRL-H key

2520 CALL Help2(Cplot)

2526 CASE 12

! CIRL-L

25326070 Braft

2538 CASE 4

! CTRL-D key

2544 60T0 Dump_to_plotter

2550 CASE $16,-78$

! CIRL-P, OUHP GRPPHICS

2556 G0TO Print_plot

2562 CASE 19

! CTRL-S

2568 GOSUB Pen_speed

25746070 React2

2580 CASE 5

! CTRL-E

2586 If Slope THEN Error_enuelope

2592 CASE 6

! CTRL-F

2598 GOSUB Force_inter

2604 If RS $(>* *$ THEN Mpts

2610 CASE 32 10 122,-67 ! Valid alphanumeric key for plotting

2616 Clear I or ConIINUE key

2622 Symbol=5

2628 If Keycode $=-67$ THEN I (CONIINUE key- use + as symbal)

2634 Keys $="+"$

2640 ELSE

2646 Keys=UPCs (CHRs(Keycode))! valid alphanumeric key

2652 END If

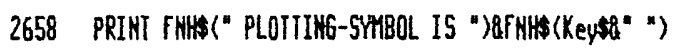

26646010 Input_data

2670 CASE ELSE

! invalid key

$2676 \quad 60101752$

2682 END SELLCT

26886010 More_points

2694 !

2700 force_inter:L00P ! force the next Yorkfit through an arbitrary age on the concordia curve

2706 Clear

2712 OFF KEY

2718 PRIHT TRBXY $(4,12)$; "Uhat age (in Ma) do you want to force the Yorkfit through?"

2724 OH KEY 9 LABEL " ESCAPE" GOTO 2880

2730 ON KBD 60102748

2736 DISP "?"

274260702742

$2748 \quad k=F$ NGet_key

2754 OfF KBO

2760 OfF KEY

2766 If K(NUH("O") OR KXWUH("g") THEN 2724

2772 DUTPUT KBD; CHRS (K);

2778 DISP FNAsts;

2784 INPUT RS 
2790 If RS $=$ "*" THEN RETURN

2796 ON ERROR $60 T 02832$

2802 Torced_age=UAL (iRS)

2808 If Forced_age=0 ThEN forced_age=1.E-8 ! else get divide-by-zero errors

2814 OFF ERROR

2820 EXIT If Forced_age $(6000$ OR Forced_age $)-2000$

2826 OFF ERROR

2832 Bad_input("InURLIQ FORCEQ-RGE ("QRRG")")

2838 END LOOP

$2844 \quad !$

2850 Clear

2856 OISP FNHS("FORCE NEXT YORKFIT THROUGH "gRSR" na")

2862 BEEP

2868 UAIT 1

2874 forced_inter=1

2880 RETURN

2886 !

2892 Input_data:0IT KEY ! request user to enter his data-points for plotting

2898 OFF KBD

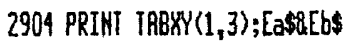

2910 If ofile IHEW

2916 PRIHT "IMPUT DATAFILE SEI Is AS 1st SET, LAST SET-ASTERISK (e.g. 2,12* or 2,12:15,18*)"

2922 PRINT USING "K, $/$ "; "Luse * $L$ or *R to label data-file name to (L)eft or ( $R$ )ight of point]"

2928 ENO IF

2934 Data_request:605UB Input_points

2940 oN 1+(Ii)Haxnum) 6010 Data_request, Edit

2946 !

2952 Input_points:0ATA 0,0,0,0,0

2958 RESTORE Input_points

2964 READ Xu(Ii),Yu(Ii),Ex(Ii),Ey(Ii),RO(Ii)

2970 Alpha

2976 OFF KEY

2982 If I $i=1$ THEX

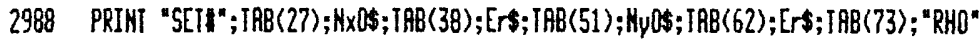

2994 PRINT

3000 END If

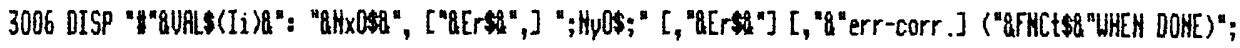

3012 O_kbd:ON K80 60103036

3018 OH KEY 9 LABEL " ESCAPE" GOTO More_points

3024 6010 3024

3030 !

3036 K=FNGet_key

3042 OFF KBD

3048 ofF KEY

3054 SELECT $K$

3060 CASE -63 ! RECALL Key

3066 OUTPUT KBD; CHRS (255) BCHRS (63);

307260703090

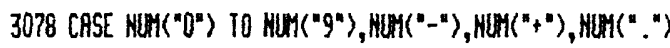

3084 OUTPUT KBD;CHRS (K);

3090 LINPUT Res

3096 If Res $=" * *^{2}$ THEE More_points

3102 CASE -67 ! CONITNUE

$3108 \quad 6070$ Edit

3114 CASE ELSE

31206010 __kbd

3126 ENO SELECT

3132 !

3138 ! If datafile input, use comas to separate data-sets in a continuous series, senicolons to separate individual sets or differe int series. 
3144 ! so 2,$5 ; 7 ; 9,14 ; 17,29 ; 6 *$ indicates sets 2 thru 5 plus set 7 plus sets 9 thru 14 plus sets 17 thru 29 plus set 6 .

$3150 !$

3156 Process_dstring:Semicolon=P0S(Res, ":") I Semicolon in data-input strung?

3162 If Sericolon THEM

$3168 R S=R e s[1$, Senticolon-1]

3174 ELSE

3180 RS $=$ Res

3186 ENO If

3192 Retrieve(Rs, Hu(*), Hinputs, 0)

3198 If Hinputs=0 THEN Edit

3204 !

3210 If NOI POS(Res, " $*$ ) THEN

3216 of $=0$

3222 605UB Keyboardinput

3228 RETURN

3234 ELSE

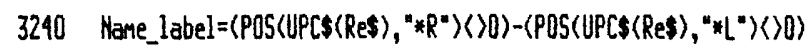

3246 ! label sanple-names to $(R\rangle$ ight or (L)eft of point

3252 END If

3258 !

3264 If NOT Ofile AND $\left(U_{c}(1,1)+U_{c}(1,3)=0\right)$ THEN

3270 Bad_input("SORRY- YOU HAUEN'T LOAOED A DATARILE INTO MEMORY")

3276 END If

3282 !

3288 of $=1$

$3294 S 1=H(1) \quad$ ! 1st, last datafile set $\| s$ in a continuous series

$330052=H v(1+($ Hinputs $>1))$

3306 !

3312 FOR Id=51 IO 52 ! Iransfer datafile sets to plotting array \& display

3318 ON ERROR 6OTO Rextid

$3324 \quad \mathrm{Xu}(\mathrm{Ii})=\mathrm{Uc}_{c}(\mathrm{Id}, 1)$

$3330 \quad \mathrm{Ex}(\mathrm{Ii})=\mathrm{Uc}_{\mathrm{c}}(\mathrm{Id}, 2)$

$3336 \quad Y_{U}(I i)=U c(I d, 3)$

$3342 \quad E y(I i)=U c(I d, 4)$

$3348 \quad R O(I i)=V_{c}(I d, 5)$

3354 If Xu(Ii)*Yu(Ii) THEN ! bypass datafile sets that aren't nonzero

3360 FOR $P=1$ TO Prtr

3366 PRINTER IS $\operatorname{Pr}(P)$

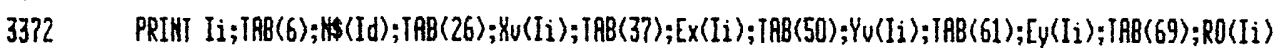

3378 NEXI $P$

3384 PRIMTER IS CRT

3390 If Ii $\langle=50$ THEN L__r $(\mathrm{Ii})=$ Kane__label

3396 If Ii $=50$ ANO Nane_label THEH Snanes(Ii)=W(Id)

3402 GOSUB Process_data

3408 If Ii=thaxnum THEN Edit

3414 ENO IF

3420 NextideNEXI Id

3426 OFT ERROR

3432 PRINT

3438 If Senicolon=0 THEN RETURH

3444 !

$3450 \operatorname{Res}=\operatorname{ReS} 5[1+$ Senicolon] I get ready to process next el enent of data-string

34566010 Process_dstring

3462 !

3468 Keyboard_input:Keyboard=1 ! Get typed-in data from keyboard

3474 If Ii $\left\langle=50\right.$ iHEN $L_{-} r(\mathrm{Ii})=0$

3480 SELECT Ninputs

3486 CASE $(2,3,15$

3492 CALL Bad_input(Cruds)

34986050 Input_points 
3504 CASC 2 I 2 values entered: $\Rightarrow x$-value and $y$-value

$3510 \quad \mathrm{Ku}(\mathrm{Ii})=\mathrm{Nu}(1)$

$3516 \quad \mathrm{Yu}_{\mathrm{U}}(\mathrm{Ii})=\mathrm{Hu}_{\mathrm{u}}(2)$

35226010 Process_data

3528 CAS[ 4,5 ! 4 or values entered: $\Rightarrow x, x$-error, $y, y$-errorl, rho]

$3534 \quad \mathrm{Ku}(\mathrm{Ii})=\mathrm{Ku}(\mathrm{I})$

$3540 \quad \mathrm{Ex}(\mathrm{Ii})=\mathrm{Hu}(2)$

$3546 \mathrm{Yu}(\mathrm{Ii})=\mathrm{Hu}(3)$

3552 Ey $(\mathrm{Ii})=\mathrm{Hu}(4)$

3558 If Ninputs=5 THEN RO(Ii)=Hu(5)

3564 END SELECT

3570 !

3576 Process_data: ! check data for nonzero $\&$ \& Y values, for permissible

3582

! error correlations. Convert errors to abs-2-signa

3588 If $\mathrm{Xu}(\mathrm{Ii}) \times \mathrm{Pu}(\mathrm{Ii})=0$ THEN

3594 Clunk

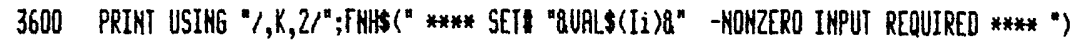

3606 RETURN

3612 END IF

3618 If Percent THEN

$3624 \quad \mathrm{E}_{-}=\mathrm{Ex}(\mathrm{Ii}) \quad$ ! percent or fractional errors for rho-testing

$3630 \quad E y_{-}=E y(I i) \quad$ I ditto

3636 ELSE

$3642 \quad \mathrm{x}_{-}=\left[\mathrm{x}(\mathrm{Ii}) / \mathrm{Xu}_{\mathrm{u}}(\mathrm{Ii}) * 100 !\right.$ ditto

$3648 \quad\left[y_{-}=E y\left(I_{i}\right) / N_{0}(I i) * 100 !\right.$ ditto

3654 END If

3660 Check_rho(RO(Ii), Ex(Ii), [y(Ii), Yu(Ii),Yu(Ii),Percent, Ok)

3666 If Ok THEN

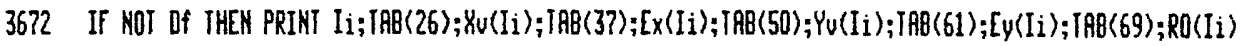

$3678 \quad \mathrm{Ii}=\mathrm{Ii}+1$

$3684 \quad N=N+1$

3690 END IF

3696 RETURN

3702 !

3708 !

3714 [dit: ! allow user to edit/correct keyboard-entered data

3720 If Keyboard THEH

3726 Clear

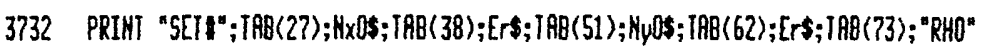

3738 PRINT

3744 FOR I=Kk TO N

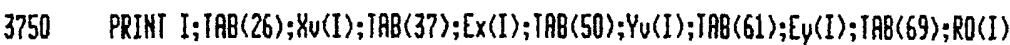

3756 NEXT I

3762 PRINT

3768 END IF

3774 !

3780 If HOT Keyboard AND Of AND Senicolon IHEM Plot_points

3786 If ABS(Symbol) $>3$ a AlO Symbol<>5 THEN

3792 ! insist on assigned errors for error-ellipse/box/cross plot-synbols

3798 Missine errors:Missing errors $=0$

3804 FOR I $=$ Kk IO H

3810 Missing errors:thissing errors $+(E x\langle I) * E y(I)=0)$

3816 NEXI I

3822 If Missing_errors IHEN

$3828 \quad$ LOOP

$3834 \quad$ OISP

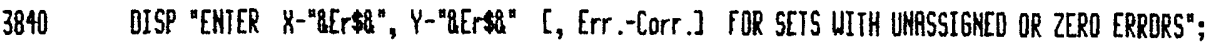

3846 Retrieve(Rs, Nu(*), Ninputs)

3852 [XII If Ninputs>1

$3858 \quad$ CRLL Bad_input(Crud\$) 


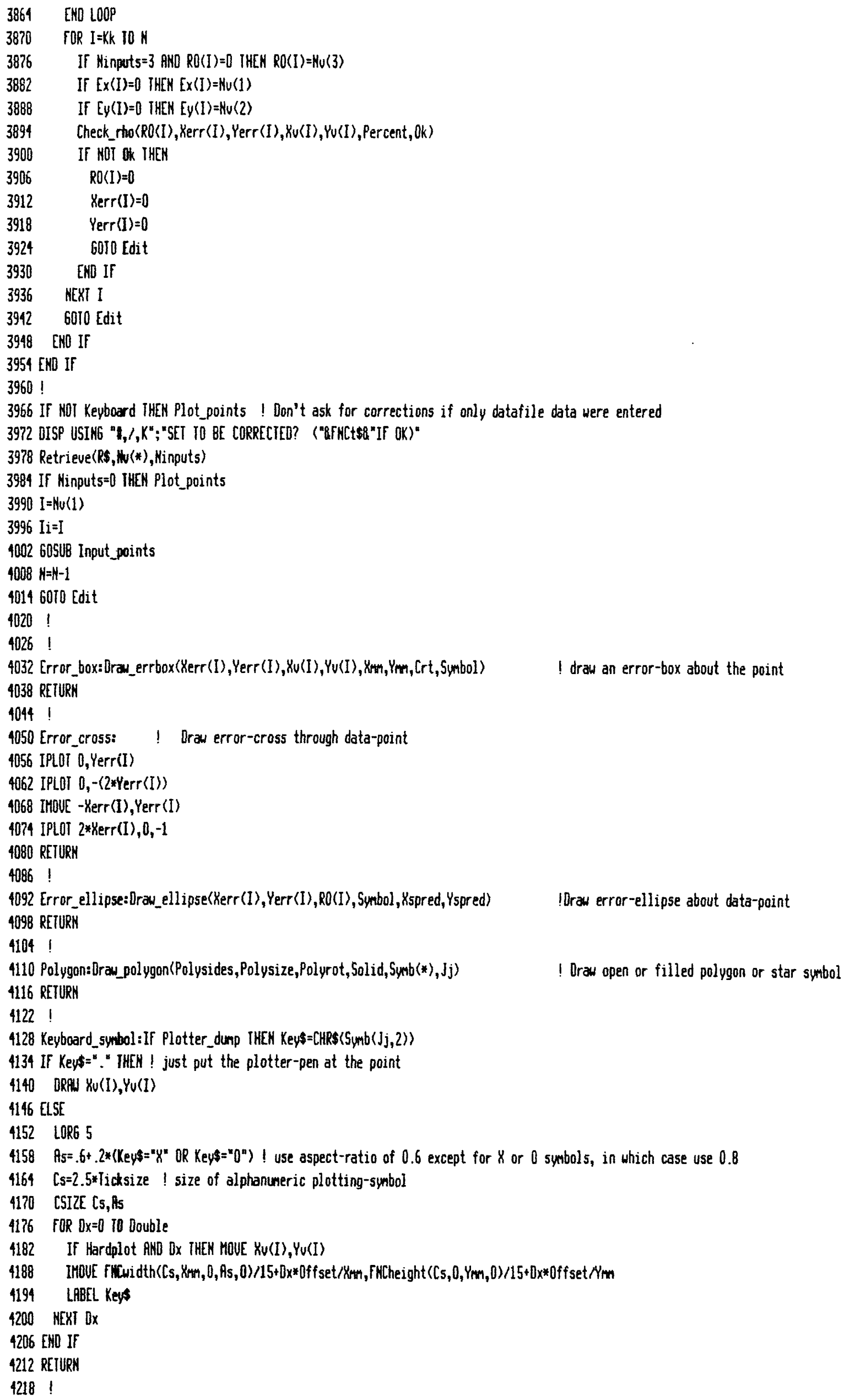




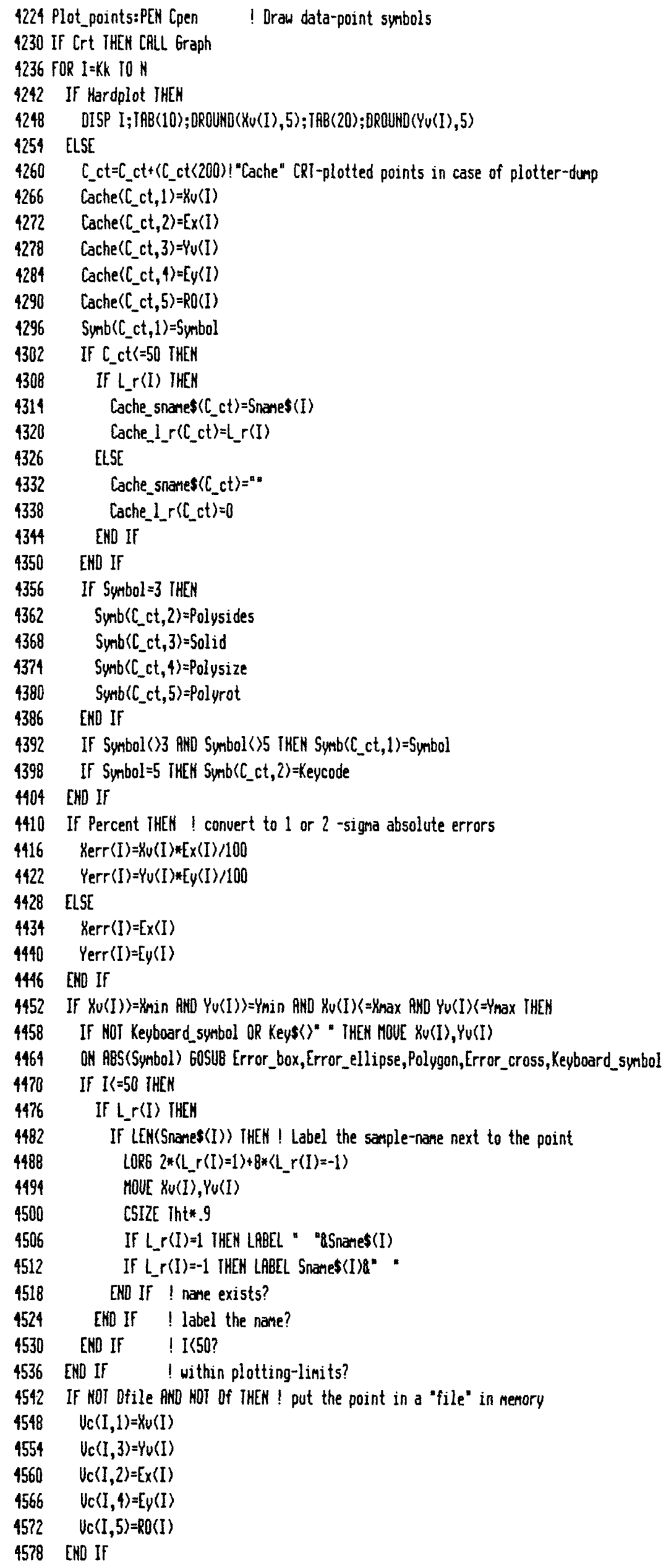


4584 MEXI I

4590 Somepoints=1 1 indicates that at least 1 point has been plotted

4596 If HOT Plotter_dump THEN

4602 Mour Xmin, Ymax

4608 If Hardplot IHEN PEN O

4614 [LSE

4620 RETURN

4626 END If

$4632 !$

$4638 \quad$

4644 Add:Clear ! Yorkfit, or plot more points?

4650 PRINTER IS CRT ! Just in case

4656 If Crt THEN

4662 Alpha

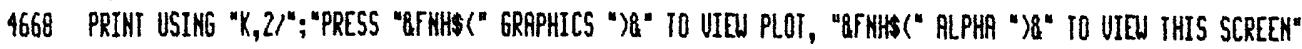

4674 END If

$4680 \mathrm{Hn}=\mathrm{H}$

4686 ON OELAY 3600 6010 Redraw I If no user action within an hour, restart.

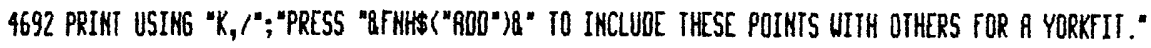

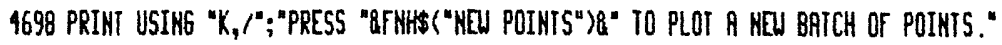

4704 IF N>1 THEN PRINT USING "K, 2 "; "PRESS "QFFHHS("YORKFII")8" FOR YORKFIT."

4710 PRINT USING "K, $/$ "; "PRESS "QFHHS ("HEU PLOT" $>$ " TO STARI A NEU PLOT."

4716 If Cplot AND N THEN PRINT USING "/, $\mathrm{K} " ;$ "press "BFFHS("CTRL-F")8" to force the Yorkfit line through a point on concordia"

4722 PRINT USING "K, $"$ ";"PRESS "8FHHS("CTRL L")8" TO ORPFT A PHRASE ON thE PLOT"

4728 DISP "(press "8FHHS("NID POINTS") 8 " if you just want to return to the data-entry screen)"

4734 !

4740 Readd: OH KEY O LABEL " HELP“ 6010 Call_help4

4746 If Nh/1 THEN OH KEY 2 LABEL - YORKFIT" GOTO Call_yorkfit

4752 ON KEY 3 LABEL " AOO POINTS" GOTO Retain

4758 ON KEY 8 LABEL "OLLETE POINTS" 60704950

4764 OH KEY 4 LABEL - NEU POINTS" GOTO Plotdata

4770 ON KEY 9 LABEL " NEN PLOI" GOTO Redraw

4776 Add_kbd: ON KBD GOTO 4812

478260104782

4788 !

4794 Adlet:Letter (Letter $\$(*)$, Letter (*), Nphrases, 0)

48006010 Add

4806 !

$4812 \mathrm{~K}=$ FNGet_key

4818 OFF KBD

4824 OFF KEY

4830 SELECT $K$

4836 CASE 8

! CTRL-H

4842 GoTo Call help4

4843 CASE 5 ! CIRL-E

4844 If Slope THEN Error_envelope

4848 CASE -67

! COHIINUE key

4854 600 Plotdata

4860 CASE 12

! CIRL-L

$4866 \quad 6010$ Adlet

4872 CASE 6

! CTRL-F

4878 60SUB Force_inter

4884 If $R S=* *$ " THEN Add

48906010 Call_yorkfit

4896 END SELECT

49026070 Readd

4908 !

4914 Retain:0Ff KEY ! Add next points to existing sequence

4920 off KBD

$4926 \mathrm{Ii}=\mathrm{N}+1$ 
$4932 k k=k+1$

4938 G0T0 More_points

4944 !

4950 60SUB Delete_points

49566070 Add

4962 !

4968 Options: Clear

4974 ofF KEY

4980 off KBD

4986 Alpha

4992 Reopt: PRINI IABXY(1,17); FNCenters(FHHS("HISCELLANEOUS OPIIONS: "))

4998 DISP FNCenters("(Press "BFHHS("CTRL-H")8" for HELP)")

5004 ON KEY O LABEL " LOAO PLOT" GOTO LoOd_plot

5010 ON KEY 1 LABCL " ABS ERRORS" GOSUB Ab__errs

5016 OH KEY 2 LRBEL " " ERRORS" 60SUB Percent_errs

5022 ON KEY 3 LABEL " 1-SI6 ERRORS" GOSUB One_sig

5028 OH KEY 4 LABEL " 2-SIG ERRORS" GOSUB Two_sig

5034 ON KEY 9 LABEL " ESCRPE" GOTO More_points

5040 If Crt IHEN ON KEY 5 LABEL " STORE PLOT" GOTO Store_plot

5046 If Cplot=0 THEE

5052 ON KEY 6 LABEL - EXPO FII" GOTO Exp_fit

5058 ON KEY ? LPBEL " LOO FII" 60TO Lo fit

5064 ON KEY 8 LABEL " POLYFII" GOTO Regress_poly

5070 END IF

5076 OH KBD 60105094

$508260 T 05082$

5088 !

$5094 \mathrm{~K}=$ FNGet_key

5100 off KBD

5106 SELECT $K$

5112 CASE 8

! CTRL-H

5118 G0To Call_help3

5124 CASE -67

! CONIINUE

51306010 More_points

5136 END SELECT

51426070 Reopt

5140 !

5154 Abs_errs: Percent $=0$

5160 Ebs=" ABS. ERRORS"

5166 PRINT EbS

5172 BEEP

5178 Ers $=$ "err"

51846010 Kretrn

5190 !

5196 Percent_errs:Percent=1

5202 Ebs=" "

5208 PRINT Ebs

5214 BEEP

5220 Er $\$="$ "Yerr"

52266050 Kretrn

5232 !

5238 One_sig: $S i g=1$

5244 BEEP

5250 Eas $=" 1-5 I$ GHA"

5256 PRINT EasR" ERRORS"

52626070 Kretrn

5268 !

5274 Two_sig:Sig $=2$

5280 BEEP

5286 Eas $s=2-S I G H A "$ 
5292 PRINT East" ERRORS"

52986070 Kretrn

5304 !

5310 Store_plot:0FF KEY

5316 off KBD

5322 Alpha

5328 Clear

5334 INPUT "ENTER A FILE-NMME FOR THIS PLOT (* to escape)?",6files

5340 If Gfiles $(>)^{*} *$ THEW

5346 DISP "Storing CRT plot on disk as file "BCHRS(34)\&GfilesRCHRS(34)8"..."

5352 GSTORE Crt_plot $(*)$

5358 OH ERROR GOTO Bad_gfile

5364 CREATE BDRF 6files, $7500 / 256+100$

5370 ASSIGN BPathl 10 Gfiles

5376 OUTPUT QPath1;Crt_plot(*)

5382 ASSiGN QPathl to *

5388 DISP "GRAPHICS-FILE "BCHRS(34)86filessChrs (34)8" STORED."

5394 OFf ERROR

5400 END If

54066010 More_points

5412 !

5418 Bad_gfile:OFf ERROR

5424 DISP FNCenters(FHHS("*** UNABLL TO ACCESS GRPPHICS-FILE "Q6filet8" ***"))

5430 CAT

5436 Clunk

5442 UAII 3

54486070 More_points

5454 !

5460 Load_plot:0FF KEY

5466 OFF KBD

5472 Clear

5478 flipha

5484 PRINT TABUY (1,12); "LhAT IS THE NRAE OF THE GRAPHICS-FILE ThAT YOU UANT TO LORD (* to escape)?*

5490 INPUI 6files

5496 If Gfiles \langle\rangle$^{* * *}$ THEN

5502 Clear

5508 DISP "Laading graphics file "RCHPS(34)\&GfilesBChRs(34)8"..."

5514 ON ERROR 6070 Bad_gfile

5520 RSSIGN QPathl 10 Gfiles

5526 ENTER PPathi; Crt_plot(*)

5532 Graph

5538 6LOORO Crt_plot $(*)$

5544 off ERROR

5550 OISP

5556 UAII 2

5562 ENO If

5568 G0T0 More_points

5574 !

5580 Call_yorkfit:CALL Yorkfit(Cplot,forced_inter, Forced_age)

5586 ON DELAN $360060 T 0$ Redray ! If no user action within an hour, restart.

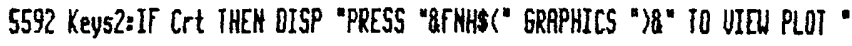

5598 off KEY

5604 ON KEY 9 LABEL " NEL PLOT" GOTO Redraw

5610 ON KEY 3 LABEL " ADO POINTS" GOTO Retain

5616 ON KEY 8 LRBEL "OELETE POINTS" GOTO 5814

5622 ON KEY O LABEL " HELP" GOTO Call_help6

5628 ON KEY 4 LABEL " CONTINUE" GOTO Plotdata

5634 ON KEY 5 LABEL "ERROR EMUELOPE" GOTO Error_envelope

5640 ON KBD $60 T 05658$

564660105646 
$5652 !$

$5658 \mathrm{~K}=\mathrm{F}$ Noet_key

5664 off KBD

5670 SELECT K

5676 CASE 8

! CTRL-H

56926010 Call_help

5688 CASE -6 ?

! COHIINUE key

56946010 Plotdata

5695 CASE 5

56966010 Error_enuelope

5700 END SELECT

570660105604

5712 !

5718 Error_envelope:PEH Cpen

5724 If Crt IHEN CALL Graph

5730 Err_enu(Slope, Errs],Inter,Errint, X_Lar, $Y_{\text {min }}, Y_{\text {max }}, Y_{\text {min }}, Y_{\text {max }}$ )

57366070 Plotdata

5742 !

5748 Print_plot:0FF KEY

5754 Clear

5760 off KBD

5790 DUAP GRAPHICS

58026010 More_points

5808 !

5814 60SUB Delete_points

58206010 Keys2

$5826 !$

5832 Delete_points: OFF KEY

5838 off KBD

5844 Clear

5850 PRINTER IS CRT

5956 PRINT "SETR"; TA8(20); HXOS; TAB(35); NyCS

5862 FOR I=1 io $\mathrm{Nn}$

5868 PRINT I; TAB (19);XU(I);TAB(34);YU(I)

5874 KEXI I

$5890 I=0$

5886 OISP "UHICH SET DO YOU UANT TO OELLTE? ("QFNCts\&"to escape)";

5892 INPUI I

5898 OISP

5904 If IL1 OR I\Wn IHEN Keys2

$5910 \mathrm{Hn}=\mathrm{Hn}-1$

$5916 \mathrm{~N}=\mathrm{H}-1$

5922 fOR J $=I$ TO H

$5928 \quad X_{u}(J)=X_{u}(J+1)$

$5934 \quad Y_{u}(J)=Y_{v}(J+1)$

$5940 \quad[x(J)=[x(J+1)$

$5946 E y(J)=E y(J+1)$

$5952 R O(J)=R O(J+1)$

5958 MEXI J

5964 RETURN

$5970 \quad$ !

5976 OraftaLetter (Letter\$(*), Letter (*), Nphrases, 0)

5982 60io More_points

5988 !

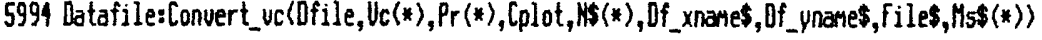

$6000 \mathrm{Hx} \$=\mathrm{Df}$ _xnares

$6006 \mathrm{Ny} \$=0 f_{\text {_ }}$ ynames

6012 RETURH

6018 !

6024 Regress_poly:If Nn)2 IHEN 
6030 CALL Polynomial (Xuv(*),Yu(*), Hn, Maxnum, Xmin,Xmax, Xspred, Cpen, Crt, Pr(*),Prtr)

6036 ELSE

6042 GRAPHICS OFF

6048 CRLL Bad_input("NEED AT LERST 3 POINTS fOR POLYHOHIAL REGRESSION")

6054 END If

60606070 More_points

6066 !

6072 Dunp_to_plotter:flipha I Iransfer CRT-plot to plotter

6078 Plotter_ok=1

6084 ON IIMEDUT $7, .1$ CALL Plotter_timeout

6090 PLOTIER IS Plotdeu(2), "HPGL."

6096 If NOI Plotter_ok THEN More_points

6102 !

6108 OFF IIMEDUT

6114 DRTA 1, US, $1,1,0,705$

6120 RESTORE 6114

6126 READ Cpen,Speeds,Plotter_dump,Hardplot, Crt,Plotter

6132 CALL Plotbox(Cruds, [plot, $0,0,0,0$ )

6138 If Cplot THEN CALL Concordia ! draw \& label concordia

6144 Clear

6150 FOR Jj=1 TO $C_{-}$ct

$6156 k=1$

$6162 \quad N=1$

$6168 X u(K)=[a c h e(J j, 1)$

$6174 \quad Y u(K)=[a c h e(\mathrm{Jj}, 3)$

$6180[\mathrm{Ex}(\mathrm{K})=[\mathrm{a}$ ache $\langle(\mathrm{J}, 2)$

$6186 \quad E y(K)=[a c h e(J j, 4)$

$6192 R O(K)=[a c h e(J j, 5)$

6198 Symbol $=S y n b(J j, 1)$

6204 Snares $(K)=$ Cache__nare $\$(\mathrm{Jj})$

6210 If $\mathrm{Jj}\langle 50$ iHEN

6216 Snawes $(K)=$ [ache_snames $(\mathrm{Jj})$

$6222 L_{-} r(K)=$ Cache___ $r(\mathrm{Jj})$

6228 ELSE

6234 Snanes $(K)==$

$6240 \quad L_{-} r(K)=0$

6246 END IF

6252 60SUB Plot_points

$6258 \mathrm{NEXT} \mathrm{Jj}$

6264 FOR I $=1$ TO HIines

6270 Slope=Line $(I, 1)$

6276 Inter=Line(I, 2)

6282 CALL Drawline(Slope, Inter, (I), Solid_line, Line(*))

6288 If CPlot AND I 14 THEN

6294 FOR K=1 TO 8

$6300 \quad$ Cint $(K)=$ Cache_cint $(I, K)$

6306 NEXT $K$

6312 CALL Label_inters(Cint(*),(I), Cache_nsud(I))

6318 END IF

6324 NEXT I

$6330 !$

6336 FOR Jj=1 TO Hphrases

6342 CRLL Letter(Letter\$(*), Letter(*), Mphrases, Jj)

6348 NEXT Jj

$6354 !$

6360 Plotter_dump $=0$

63666070 Hore_points

$6372 !$

6378 Call_help3:CALL Help3

63846010 Options 
$6390 \quad !$

6396 Call_help4:CaLLL Help4(Cplot)

64026070 hdd

6408 !

6114 Call_help6:CRLL Helpo

6420 g0to Keys2

$6426 \quad$ !

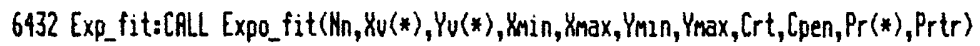

64386010 More_points

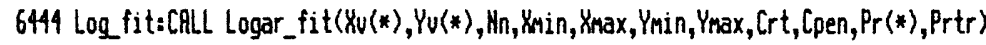

64506070 More_points

$6456 !$

6462 !

6468 ! *****************************************************************************

6474 Endmain:END ! end of main progran **********************************

$6480 !$

$6486 !$

6492 Z:DEF $F H Z(X) !$ utility function for misc. numeric formatting needs

6498 RETURH INT(LGT $\langle$ RBSS(OROUND $(X, 9\rangle+\langle X=0)\rangle)\rangle$

6504 FNEND

6510 !

$6516 !$

6522 Tick:SUB Tick(Range,Tick_interval) ！ Returns best tick-interval

6528 Tick_interval $=10^{\wedge}$ F MZ(Range) $/ 8$

6534 UHILE RBS(Range/Tick_interval) $>12$

6540 Tick_interval=2*Tick_interval

6546 END UHILE

6552 B=fBS(Tick_interval)/10^FNZ(Iick_interval)

$655 B$ If $B() I N T(B)$ THEN Tick_interval=INT (B)*10^FNZ(Tick_interval)

6564 Tick_interval=DROUND(Tick_interval,8)

6570 SUBEND

$6576 !$

$6582 !$

6588 Axis:SUB Axis(P,Spred, Tik, Ticksize,

6594 ! $x y=1$ for $x$-axis, $X y=0$ for $Y$-axis

$6600 \quad[=1-2 *(x y=0)$

6606 Ntix=DROUND(Spred/Tik,3)

6612 Etix $=$ FNEven $(N$ ti $x$ )

6618 FOR I $=1$ TO Ntix

6624 Etik=FNEven(I)

6630 If $(P=1) * E$ tik OR $\langle P=-1\rangle *(($ Etik EXOR Etix $\rangle=0\rangle$ THEN

$6636 \quad$ HeTicksize

6642 ELSE

$6648 \quad H=T i c k s i z e / 2$

6654 END IF

6660 IDRRI P*Tik*Xy,P*Tik*(NOT Xy)

6666 IQRPWU [*P*H*(NOT Ky),P*H*KYy

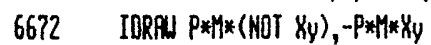

6678 NEXT I

6684 SUPEND

$6690 !$

$6696 !$

6702 De: OEF FMDe(N,B)

6708 ! Round value $N$ to $D$ significant figs.(to lowest least-sf,unlike DROUND)

$6714 \quad A=10^{\wedge}(D-F N Z(N)-1)$

$6720 B=0 R O U N D(A B S(N * A), 9)$

6726 RETURN SGK(N)*INT(B)/A

6732 FNEAD

$6738 !$

6744 ! 


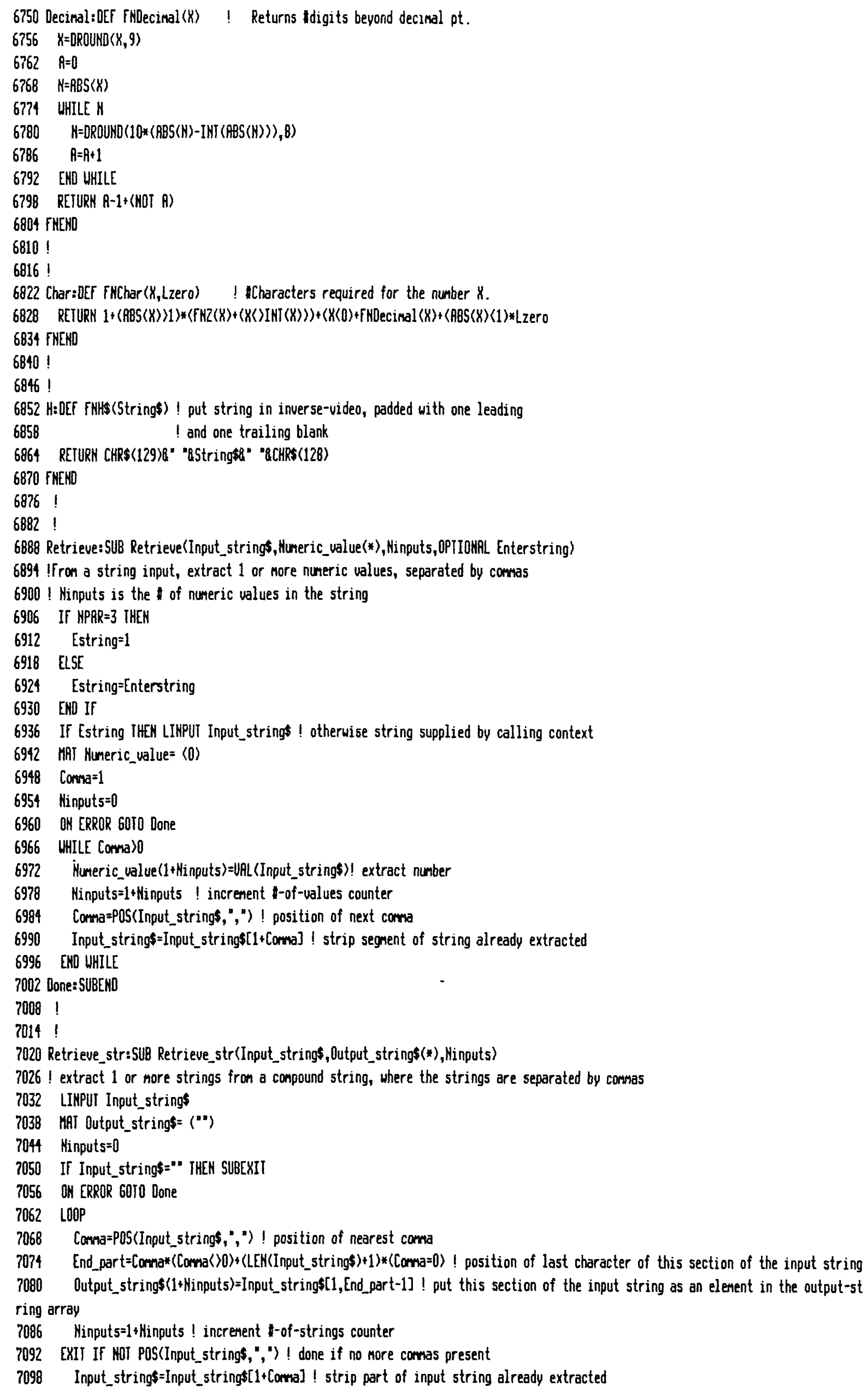


2128 Remove: SUB Remove(Input_string\$, Output_string\$, Length)

7134 ! remove super- (CIRL ") and sub- (CTRL *) script indicators from label

7140 Output_strings:"*

7146 TOR J=1 TO LEN(Input_string\$)

$2152 \quad N=$ NUtH(Input_string $\$[J, J])$

7158 SELECIN

2164 CASE 10,30

7170 ! do nothing

7176 CASE ELSE

7182 Length=1+Length

7188 Output_strings[Length, Length]=Input_strings[J,J]

7194 ENO SELECT

7200 NEXT J

7206 SUBEND

7212 !

$7218 !$

7224 Draw_ellipse:SUB Oraw_ellipse(Yerr,Yerr,RO,Symbol, Xspred,Yspred)

$7230 \quad$ Ke $_{e}=$ herr $*(1+.0001 *($ Yerr $=$ Yerr $))$

7236 Corr $=R O *(R O<>1)+.9999999 *(R 0=1)$ I Guard against a rho of 1

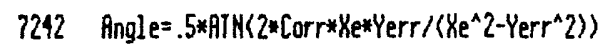

7248 ! AKGLE is an angle that the axis of the ellipse nakes with the $X$-axis

7254 C1 $22 *\left(1-\operatorname{Corr}^{\wedge} 2\right) * 1.224^{\wedge} 2$

$7260 \quad[2=1 / \cos (2 *$ *ngle)

$7266 \quad U \mathrm{x}=\mathrm{X} \mathrm{e}^{\wedge} 2$

7272 Vy=Yerr^?

$7278 A=\operatorname{AQR}\langle C 1 /((1+C 2) / N \times+(1-C 2) / N y)) \quad$ ! Length of najor axis of ellipse

$7284 B=S q R\left(C 1 /\left(\left(1-\{2) / \|_{x}+(1+C 2) /(y)\right)\right) \quad\right.$ ! Length of minor axis of ellipse

7290 St=A/IhT(A/ $/(X$ spred/400)+1) IStep-size along $X$-axis

7296 St=h/INT(A/St+.0001) Iforce step to divide evenly

7302 Sin_angle=SIN(Angle)

7308 Cos_angle=COS(Angle)

7314 FOR Seq $=1$ TO $1+\left(S_{\text {ymbol }}<0\right)$

7320 FOR $K=1$ TO -1 STEP -2

7326 Start_ $x=K *$.

7332 End_ $x=-K *(A+S t)$

$733 B \quad$ FOR $x=$ Start_ $x$ TO End $x$ STEP $-(K *$ St $)$

7344 If $(K=1) *(X(-(A) * 1.001))$ OR $(K=-1) *(X) A * 1.001)$ THEH 7428

$7350 \quad z=1-(X / A) \wedge z$

7356 If $Z>=0$ THEN

$7362 \quad Y=K * B * 5 Q R(Z)$

$7368 \quad$ ELSE

$7374 \quad Y=0$

7380 ENO If

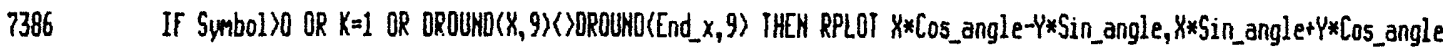

7392 If Seq=2 THEN $!$ fill in the ellipse

$7398 \quad k=-k$

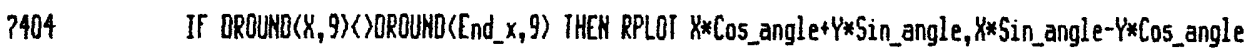

$7410 \quad$ END IF

7416 NEXT X

7422 If Seq=2 THEN SUBEXII

7428 MEXI K

7434 NEXT Seq

7440 SUBENO

$7446 !$

$7452 !$

7458 Draw_polygon:SUB Oraw_polygon(Polysides,Polysize,Polyrot,Solid,Synb(*),Jj) 
2464 ! draw a polygon of arbitrary size, Isides, orientation, infill

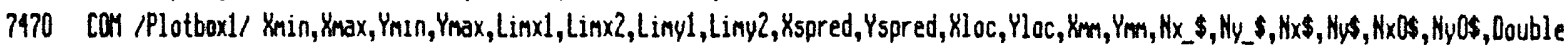

2476 COH /Plotboxla/ Lx1,Lx2,LyI,Ly2,0ffset

7482 Con /Plotbox2/ Hxr\$, Hurs,Plotdev(*),Pr(*),Plotter,Plotter_dump,Ar, Tht,Lht, Ticksize, XI,Yl,Yend, Yend, Speed\$, Crt, Hardplot, Cpen, X

gu, Ygu

7488 !

2494 If Plotter_dump IHEN ! cache polygon-specs

7500 Polysides $=5 y m b(J j, 2)$

$7506 \quad$ Solid $=$ Symb $(J \mathrm{j}, 3)$

7512 Polysize $=$ Symb $(\mathrm{Jj}, 4)$

$7518 \quad$ Polyrot $=5 y$ mb $(J j, 5)$

2524 EHO IF

$7530 \quad !$

7536 Star=(Polysides $\langle 0\rangle$ ! is this to be a "star"-shaped figure?

$754257475=$ (Hardplot ANO Solid AND NOT Star AND Polysides( $) 7$ )

7548 If 57475 IIHN ! use NP-7475 HPGL command to draw solid poly

$7554 \quad S=0$

7560 OATA $0,0,120,90,72,60,25.714,0$ ! starting=position, in degrees

7566 RESTORE 7560

7572 If Polysides 99 THEN

7578 FOR I $=1$ TO Polysides

7584 READ 5

7590 NEXT I

7596 EKD If

$7602 P=P$ olysides*(Polysides $\langle 10)+50 *(P$ olysides $\rangle=10\rangle !$ use 50 sides if polysides $\rangle 9$

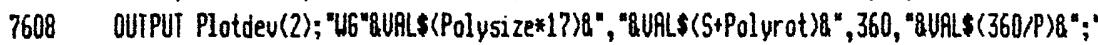

3614 ENO If

$7620 X_{p}=P o l y s i z e / X_{m} / 2.3 \quad$ I rake diameter equal to polysize in millineters

$7626 \quad Y_{p}=P_{0}$ lysize/ $/$ ma/2.3

$76320=.2+.1 \times$ Crt $\quad$ effective pen-thickness in m

7638 H_polys $=\langle 57475=0) *(($ Hardplot ANO (Ticksize(1.4) AND Polysides $>0$ AND Polysize $\rangle 3\rangle+50 * 50 l i d)$

7644 If N_polys=0 AND Double AND $57475=0$ THEN H_polys $=1$

7650 INpolys is the number of (increasingly smaller) polygons to be drawn

7656 FOR I $=0$ iO H_polys

$7662 \quad x=x_{p}-I * D / x_{m}$

$7668 \quad Y=Y p-I * \Omega / \% m$

$7674 S=360 /$ ABS (Polysides)

$7680[E=.5$-(BASSPolysides) $=3$ ) $/ 5$ ! degree of pucker-indent for star

7686 If $X<O$ OR Y YO THEN SUBEXIT ! stop then radius of zero is passed

$7692 \quad !$

7698 FOR J=Polyrot to Polyrot+360 STEP S

$7704 \quad$ RPLOT $x * \operatorname{COS}\langle\mathrm{J}\rangle, y * S I M(\mathrm{~J}\rangle$

7710 If Star IHEN ! do a pucker

$7716 \quad[=J+5 / 2$

$7722 \quad$ RPLOT $E * X * \operatorname{COS}(C), E * Y * \operatorname{SIH}(C)$

7728 EHD IF

7734 HEXI J

7740 HEXI I

7746 SUBEND

7752 !

$7758 \quad !$

7764 Oraw_errbox:SUB Oraw_errbox (Yerr, Yerr, X,Y, Xm, Ymm, Crt, Symbol)

7770 ! draw a rectangle whose dimensions correspond to the uncertainties of the point

7776 IMOUE -Kerr,-Yerr ! start at the lower-left corner of the box

$7782 \quad \omega=2 *$ Kerr ! box height

$7788 \mathrm{H}=2 *$ Yerr $\quad$ ! box width

$7794 !$

7800 ! use RECTAHGLLE command for CRT-plots or open boxes

7806 If NOT Crt OR (Crt AND Symbol>O) THEN RECTANGLE $U, H$

7812 If Crt Alid Symbol<O THEN RECTANGLE W,H,FILL 
3830 Penthick $=.36 / \mathrm{mm} /(1+C r t)$ ! UU's per $.36 \mathrm{~m}$ in Y-direction

$7836 \quad D=1$

7842 CLIP X-Xerr, X+Xerr,Y-Yerr, Y+Yerr

7848 REPEAI ! fill in the box (plotter only)

3854 IORRN $0 * \mathrm{H}, \mathrm{O}$

2860 IDRRI 0,Penthick

7866 Height=Height+Penthick

$7872 \quad 0=-0$

7878 WwIL Height $>H+2 \times$ Penthick ! quit when filled in box ( + abt $1-2$ lines)

7884 CLIP $x_{\text {min, }}$ Hax $_{\max } Y_{\min }, Y_{\max }$

7890 EHD IF

7896 SUBEND

$7902 !$

79081

7914 Yorkfit:SUB Yorkfit(Cplot, Forced_inter,Forced_age)

7920 ! line-fitting algorith nodified fron D. York, EPSL v. 5, p. 320-324, 1969

7926 OPIION BASE 1

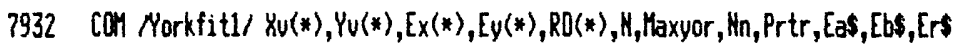

7938 CoH Norkfit2l Kerr(*),Yerr(*),Sig,Line(*), Percent, Hlines, N_interlabels, Cint(*), Solid_line, Cache_cint(*), Cache_nswd(*)

7944 CoM Norkfit3/ Slope,_Errsl, Inter,_Errint,_,_bar

7950 OIM X_ut (200),Y_ut (200),R(200), Weight(200), Mean_ut(200), Yorkslope(5), Yorkinter (5)

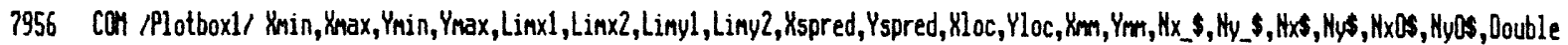

7962 Con /Plotboxla/ LxI,Lx2,Ly1,Ly2,Offset

7968 COM /PlotboxZ/ Nxr\$, Hyrs,Plotdeu(*),Pr(*),Plotter,Plotter_dump, Ar, Iht, Lht, Iicksize,Xl,Yl, Xend, Yend, Speeds, Crt, Hardplot, Cpen, X gu,Ygu

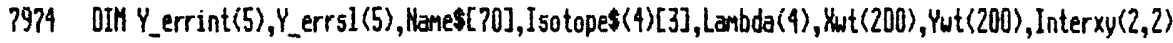

7980 OIK $E_{-} \times 0(200), E_{-} y 0(200), E_{-} 76(200), E_{-} x(200), E_{-} y(200), X_{b a r}(5), Y$ bar $(5), C_{i n t} r(8), E_{-} 760(200)$

$7986 \quad$

7992 I Rb-8?, 5m-147, U-238, U-235, Th-232 decay constants (/my)

7998 맥 1.42E-5,6.54E-6,1.55125E-4,9.8485E-4,4.9475E-5

8004 RERO Lambda(*)

8010 !

8016 If HWhaxyor IHEN

8022 Bad_input(THHS("*** CAN ONLY YORKFIT UP TO "QUhL (Maxyor)\&" POINTS ***"))

8028 SUBEXII

8034 EHD If

$8040 !$

8046 OTT KEY

8052 OFF KBD

8058 Alpha

8064 H $=$ in

8030 !

8076 ! Deternine if isochron data by looking for isotope is in $X \&$ Yames

8082 DीT 87,86,87,86,143,144,147,144,206,204,238,204,207,204,235,204,208,204,232, 204,207,204,206,204,207,206, 204,206

8088 RESTORE 8082

8094 FOR I $=1$ io ?

8100 READ Isotopes $(*)$

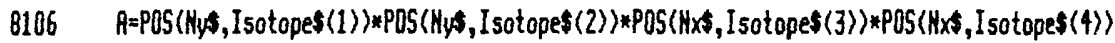

8112 If A AHB I $=5$ IHEN Isochron=I ! Iype of std isochron $(R b-5 r, 5$ st/Wd ...)

8118 If A AHA I $>=6$ IHEN Pbpb $=I-5$ ! Normal or inverse Pb-Pb isochron

8124 NEXI I

$8130 !$

8136 LODP

$8142 \quad$ Clear

8148 PRIMT USING "K,I"; "YORKFII:"

8154 ! Check for unassigned erors and error-correlations

B160 Missinq errors $=0$

8166 Zero_rhos=0 
8310

8316

8322

8328

8334

8340

8346

8352

8358

8364

8370

8376

8382

8388

8394

8400

8406

8412

8418

8424

8430

8436

8442

8448

8454

8460

8466

8472

8478

8484

8490

8496

8502

8508

8514

8520 8526
FOR I $=1$ TO N

Hissinq errors=thissinq errors $+\langle[x(I) *[y(I])=0\rangle$

If Isochron (3 OR Cplot THEN Zero_rhos=Zero_rhost $(R O(I)=0)$

! Don't ask for error-correlations for Rb/Sr or SA/Nd isochrons

NEXI I

!

If Missing errors AND NOT Zero_rhos THEN

PRINT "ZX-ERRS, "YY-ERRS FOR SPHPLES UITH UWASSIGNED OR ZERO ERRORS?"

LOOP

Retrieve(Rs, Hu(*), Hinputs)

If $R S=" *$ "THEN SUBEXIT

EXII If Hu(1) AND Nu(2)

Bad_input( "KEED AT LERST 2 MOHZERO RESPOKSES")

END LOOP

X_error=Hu(1)

Y_error=Hu(2)

[N] IF

If Missing errors ARD Zero_rhos THEN

If Percent THEN PRINT "XX-ERRS, RY-ERRS";

If NOT Percent THEN PRINT "X-ERRS, Y-ERRS";

If NOT CPlot THEN PRINT " [, ERR-CORRS]";

If Cplot THEN PRINT ", ERR-CORRS";

PRINT " FOR SRYPLES UITH UKRSSIGAED OR ZERO URLUES?"

LOOP

Retrieve(Rs, Hu(*), Hinputs)

If $R S=" *$ "THEK SUBEXIT

EXIT If Hu(1) ANO Hu(2)

Bad_input( "KEEO AI LEAST 2 MONZLRO RESPONSES")

END LOOP

X_error=Hu(1)

Y_error=Hu(2)

Rho $=$ Hu(3)

END IF

If Zero_phos AND NOT Missing errors THEN

PRIAT "ERR-CORRS FOR SFHPLES UITH UNASSIGNED OR ZERO URLUES ("QRNCTSR"IF ZERQ)?"

LOOP

Retrieve(Rs, Hu(*), Ninputs)

If $R s=*_{*}$ "THEN SUBEXIT

Rho=Hu(1)

EXII If 月⿰BS(Rho) $<=1$

Bad_input("ERROR-CORRELATOMS HUST BE BETLEEH -1 AND +1")

END LOOP

ENO If

EXIT If HOT Zero_rhos OR NOT Cplot OR Rho

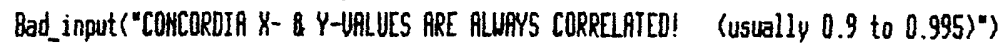

END LOOP

FOR I $=1$ TO $\mathrm{R}$

If $E x(\mathrm{I})=0$ iHEN

Ex(I) $=X_{-}$error

Xerr $(\mathrm{I})=X_{-}$_error $*($ Percent*Xu(I)/100+(Percent $\left.=0)\right)$

END If

If $E y(I)=0$ THEN

Ey $(I)=Y_{-}$error

Yerr $(I)=Y_{-}$error* $*$ Percent*Yu(I) $/ 100+($ Percent $\left.=0)\right)$

END IF

If $R O(I)=0$ THEM $R O(I)=R$ ho

NEXT I

If

If Cplot AND Forced_inter AMD HCMaxyor IHEN

$N=\$+1$ 


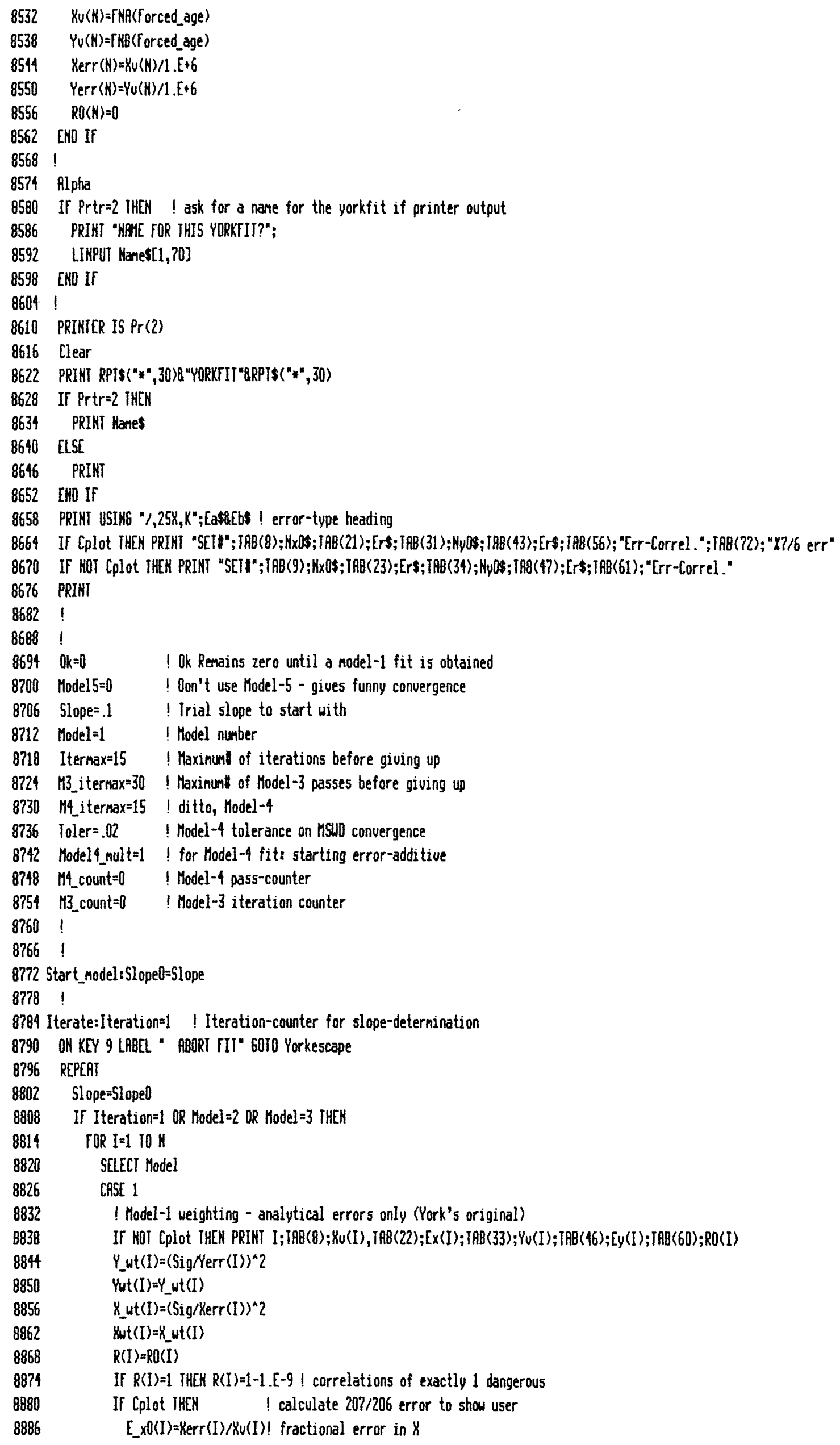




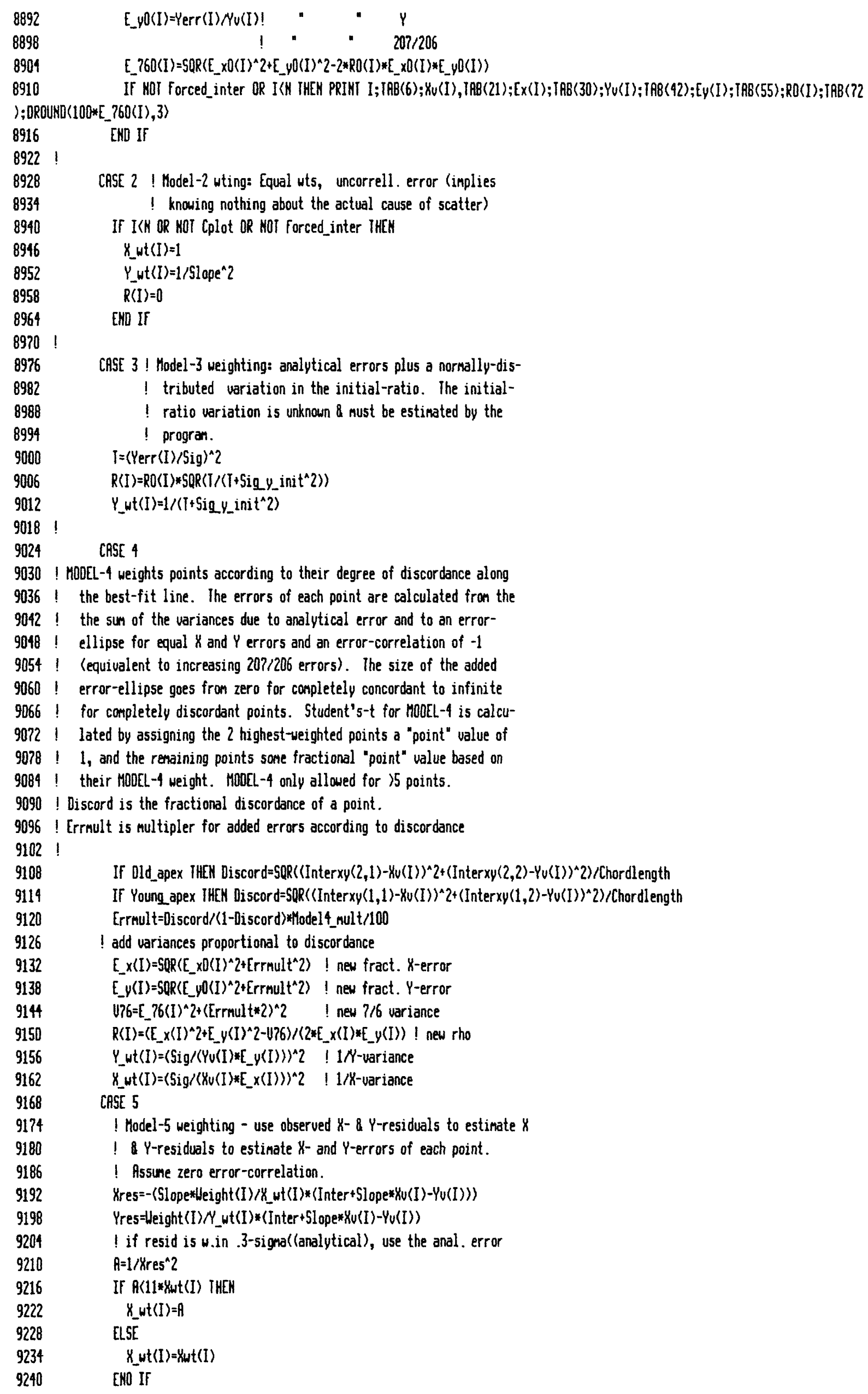

9030 I HODEL- 9 weights points according to their degree of discordance along

9036 ! the best-fit line. The errors of each point are calculated from the

9042 I the sum of the variances due to analytical error and to an error-

$9048 \div$ ellipse for equal $X$ and $Y$ errors and an error-correlation of -1

9054 ! (equivalent to increasing 207/206 errors). The size of the added

9060 ! error-ellipse goes from zero for completely concordant to infinite

9066 I for completely discordant points. Student's-t for HOOEL-4 is calcu-

9072 I lated by assigning the 2 highest-weighted points a "point" value of

9078 ! 1 , and the renaining points sone fractional "point" value based on

9084 ! their MDOEL-4 weight. MODEL-4 only allowed for 75 points.

9090 ! Discord is the fractional discordance of a point.

9096 ! Errnult is multipler for added errors according to discordance

9102 !

9108 If 01d_apex THEN Discord=5OR((Interxy $\left.\left.(2,1)-Y_{u}(I)\right)^{\wedge} 2+\left(\operatorname{Interxy}(2,2)-Y_{v}(\mathrm{I})\right)^{\wedge} 2\right) /$ Chordlength

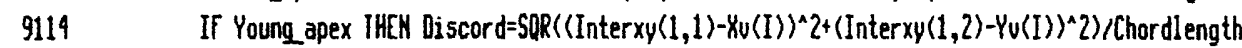

9120 Errmult=Discord/(1-Discord)*Hodel4_nult/100

$9126 \quad !$ add variances proportional to discordance

$E_{-} x(I)=S O R\left(E_{-} X D(I)^{\wedge} 2+\right.$ Errmult 2$) \quad$ ! new fract. $X$-error

$E_{-} y(I)=S Q R\left(E_{-} y 0(I)^{\wedge} 2+E\right.$ rrmult t`2) ! new fract. Y-error

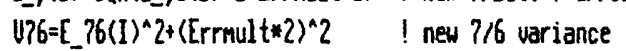

$R(I)=\left(E_{-} x(I)^{\wedge} 2+E_{-} y(I) \wedge 2-U 76\right) /\left(2 \times E_{-} x(I) \times E_{-} y(I)\right) !$ new rho

$Y_{-} w t(I)=\left(S i g /\left(Y u(I) *\left[E_{-} y(I)\right)\right)\right)^{\wedge} \mid 1 \%$-variance

$X_{-} u t(I)=\left(S i g /\left(X_{u}(I) * E_{-} x(I)\right)\right)^{\wedge} 2 \quad 1 / 1 /$-variance

CASE 5

I Model-5 weighting - use observed $X-\&$ Y-residuals to estimate $X$

I $Y$-residuals to estimate $X$ - and $Y$-errors of each point.

! Assume zero error-correlation.

Xres=-(Slope*tleight (I)/K_ut(I)*(Inter+Slope*Xu(I)-Yu(I)))

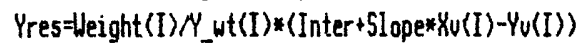

$!$ if resid is w.in .3-signa( (analytical), use the anal. error

$A=1 / 1 /$ reses $^{\wedge}$

If R(11*X)wt(I) THEN

$X_{-} w t(I)=A$

ELSE

$x_{\text {nut }}(\mathrm{I})=\mathrm{xut}_{\mathrm{u}}(\mathrm{I})$

ENO If 


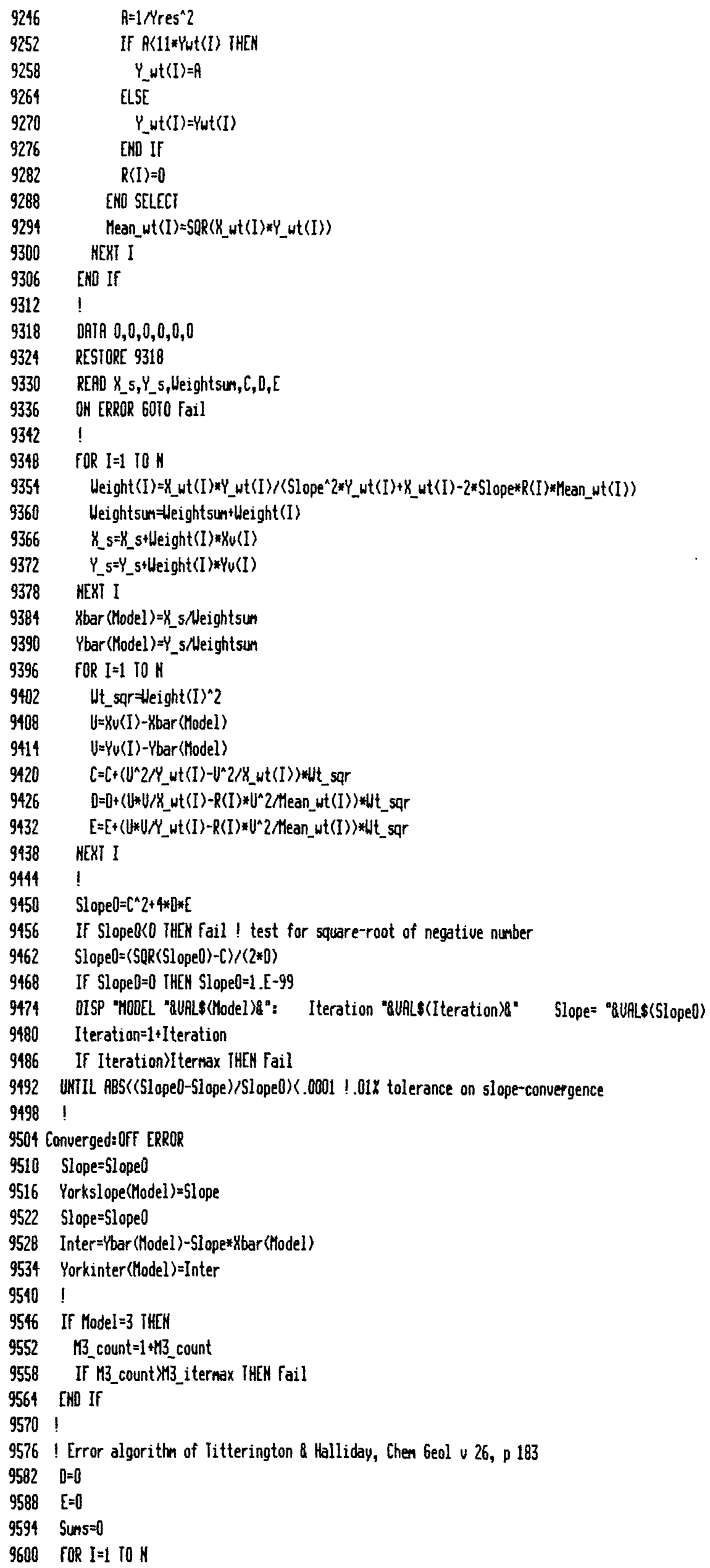




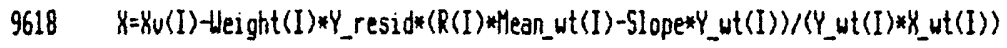

$9624 \quad D=0+K$ *bieight $(\mathrm{I})$

$9630 \quad\left[=\left[+x^{\wedge} 2 \times\right.\right.$ *leight(I)

9636 NEXT I

9642 !

9648 Nu=N-2

9654 Hul $=H u *(H u>0)+\left\langle H_{u}<=0\right\rangle$

9660 Msud $=($ Nus $)$ ) $* 5$ Surs/Nul

! Degrees of freedom

$!$ in case fitting only 23 points

9666 If Model $=4$ AND ABS(Msud-1) $)$ Toler THEN

9672 M4_count $=1+114$ _count

9678 If M4_count $744_{2}$ iternax IHEN Fail

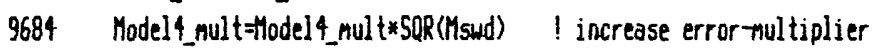

9690

OISP "Model 4: Pass ";M4_count," MSUD: ";Msud

9696 CALL Model4_calc(SIope,Inter, Cint(*), Inter xy(*), Chordlength)

97026070 Start_nodel

9708 END If

9714 !

9720 If Model $=3$ AMD ABS(Mswd-1)》.01 THEN ! increase estimated initial-ratio variation

9726 Siq_y_init=Siq_y_init*SQR (Msud) ! est. var. in init. ratio to account for scatter

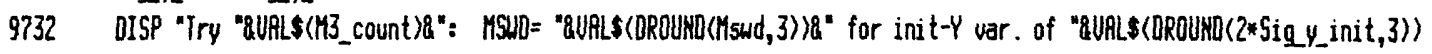

$9738 \quad 6010$ Iterate

9744 END If

$9750 \quad 1$

9756

9762

!

! Mean square of weighted deviates

9768

9774

9780

9786

9792

9798

9804

! Calculate degrees of freedon by weighting number of

If Model=4 IHEN ! points by assigning nax-utd point \& next-to-nax uted

Hcount $=1$ ! point a "point" value of 1 , and all others a lesser

Maxueight=thH(Height(*))

! value according to their yorkfit weight.

9810 FOR I=1 TO N

9816 If Weight(I)/Maxueight THEN Ncount=kicount+ Weight(I)/tax2weight

9822 NEXI I

9828 Nu=klcount-2 ! can be non-integral or $<1$

9834 ENO IF

$9840 \quad 1$

9846 Students $t=F N S t u d e n t s_{-} t(N u)$

9852 Errsl_apr=\#leightsum/(E* Leightsum-D^2)! a priori (from analytical errors only) 1-signa

9858

9864

9870

9876

9882

9888

9894

9900

9906

9912

9918

9924

9930

9936 If Model=1 IHEN ! calculate chi-square probability

9942 SELECT Msud

9948 CASE 220 ! obuiously zero-probability

$9954 \quad$ Prob $=0$

9960 CASE (.0001 I obuiously near-1 probability 


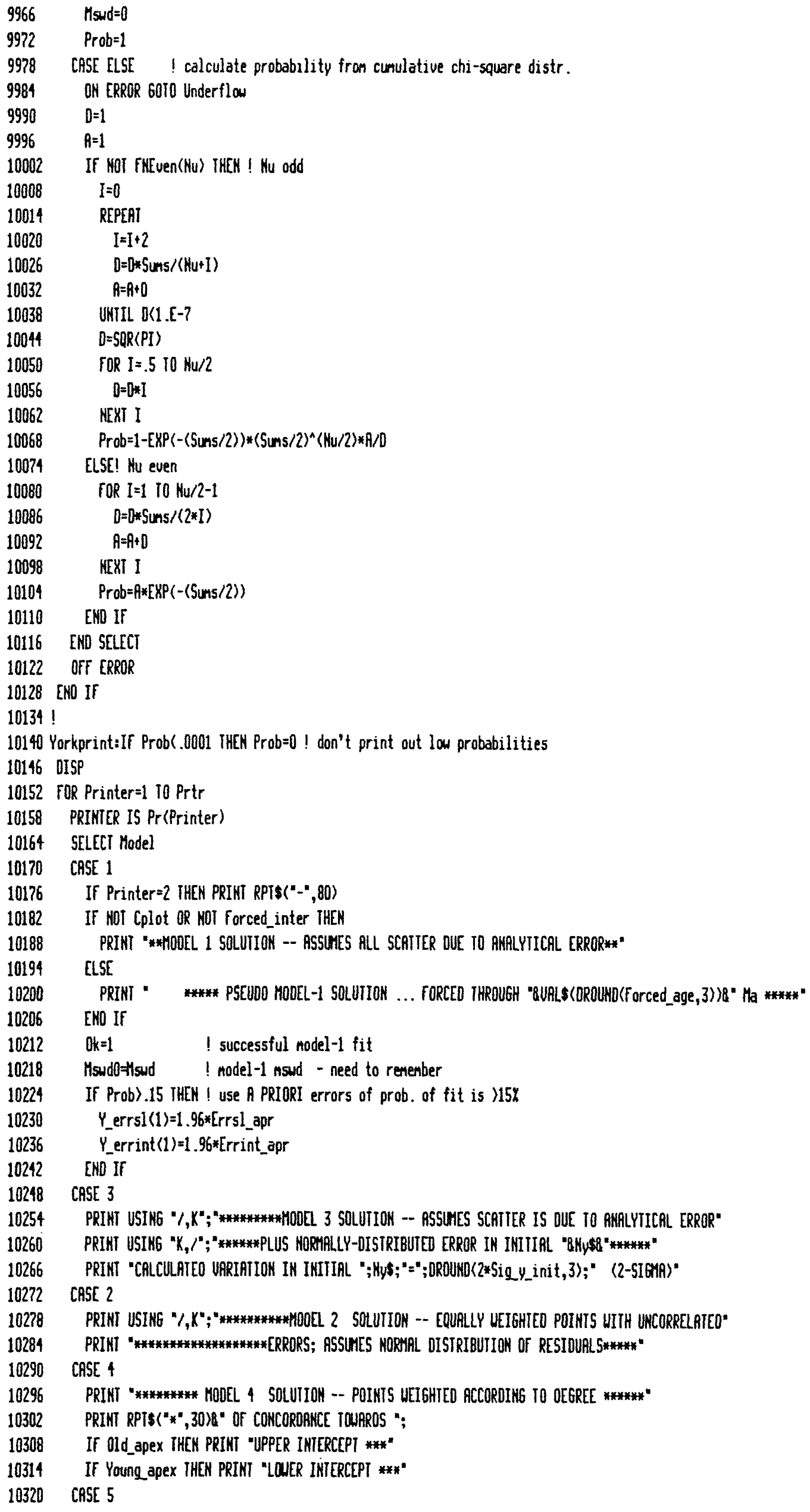



END SELECT

10338 If Model $>1$ OR Prob<=.15 THEN ! use students' $t-t$ for Hu d.f. if prob $15 \%$

10344 Y_errsl (Model) $=$ Students_t* $t$ [rrsl_incsc

$10350 \quad Y$ _errint (Model) $=$ Students__t*Erint_incsc

10356 END If

10362 If Prtr=1 ThEN PRINT USING "/,32X,X,19X,X,l"; "SLOPE", "INTERCEPT"

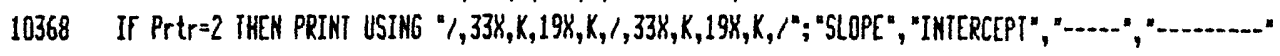

10374 PRINT "BEST-FIT:";IAB(31);OROUNO(Slope,6);IAB(56);OROUNO(Inter,6)

10380 If Printer=2 IHEN PRINT RPTS("-",80)

10386 If Model $=1$ IHEN

10392 PRINT "ERROR (1-SIGMA, A PRIORI) ";IRB(31);OROUWO(Errsl_apr, 4);ifB(56); OROUND(Errint_apr, 4)

10398 If Printer=2 THEN PRINT

10404 END IF

10410 PRINT "ERROR (1-SIGTh, FROH SCATTER) ";TAB(31);OROUHD(ErrsI_incsC,4);TAB(56);0ROUNO(Errint_incsc,4)

10416 If Printer=2 THEN PRINT

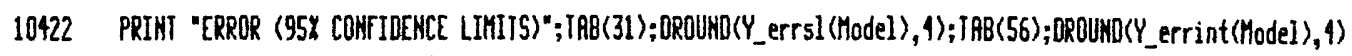

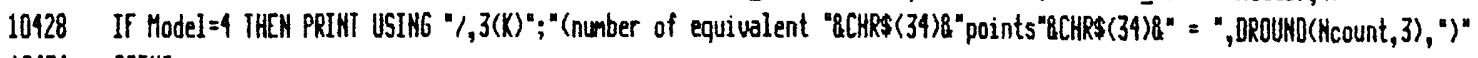

10434 PRIHI

10440 PRINT "COORUIHATES of CENTROID: $X="$;OROWHQ(Xbar(Model),6), $Y="$;OROUND(Ybar (Model),6)

10446 If Model $=1$ THEN

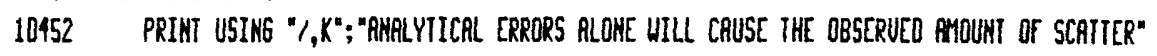

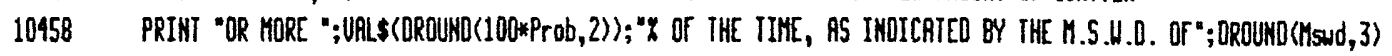

10464 [ND IF

10470 If Printer $=2$ THEN PRINT USING " $\mathrm{K}, /^{\prime \prime} ; \mathrm{RPTS}\left({ }^{*}-", 80\right)$

10476 NEXI Printer

10482 PRINIER IS CRT

10488 If (Model=1 AWO Prob =.2) IHEN I may want to try another nodel

10494 If Cplot $=0$ OR Forced_inter THEN

10500 ! Iry another nodel, since Model-1 doesn't seen too probable

10506 Model $=2+($ Isochron $>0) !$ use model -2 if non-isochron, 3 if isochron

10512 Sig_y_init=Errint_incsc ! initial est. Y-variability=intercept-error

10518 PRINT RPIS(" ",80)

10524 DISP "MODEL "8UBLLS(Hodel)\&" yORKFIT ..."

$10530 \quad 6070$ Iterate

10536 ELSE ! give user a choice of not trying another nodel

10542 OISP "ACCEPT MOOLL-1 FII OR FIT UITH ANOTHER MOOEL?"

10548 OFF KEY

10554 ON KEY 1 LABEL "ACCEPT MODEL-1" 6070 Model_chosen

10560 OH KEY 2 LABEL "DO MODELL"2" 607010698

10566 IF N)5 IHEN

10572 ON KEY 3 LRBEL "DO MBOEL-4 UI" 60TO 10716

10578 ON KEY 4 LRBEL "OO MODEL-4 LI" GOTO 10740

10584 END If

10590 OH KEY 7 LABEL" HELP" GOTO Call_help?

10596 ON KEY 9 LABEL " ESCAPE" GOTO Yorkexit

10602 ON K8D 601010620

$10608 \quad 607010608$

$10614 \quad !$

$10620 \quad k=F M G e t$ Key

10626 OFF KBD

10632 SELECT K

10638 CRSE -67 ! CONIINUE

10644 60T0 Model_chosen

10650 CASE 8 I CTRL-H

10656 G070 Call_help?

10662 END SELECT

$10668 \quad 607010542$

10674 !

10680 Call_help7:CALL Help? 


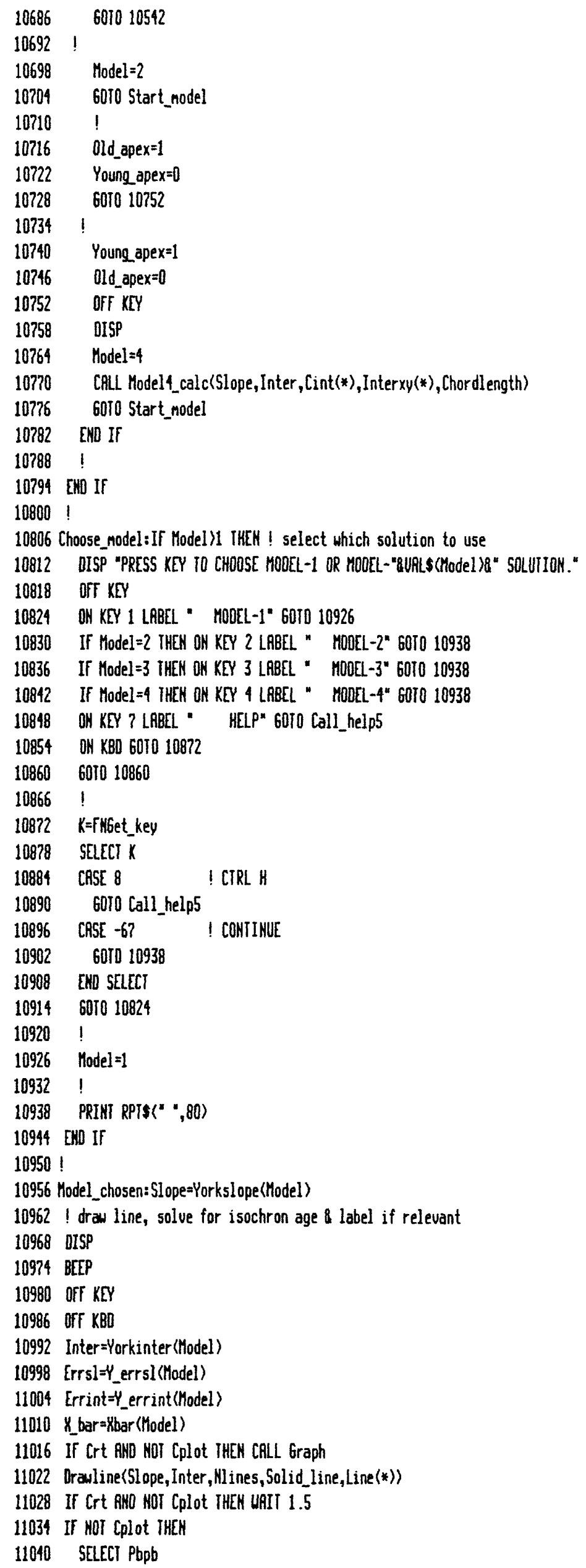


11052 If Isochron THEN

11058 fige=L0G(1+Slope)/Lambda(Isochr on)! standard-i sochron age

11064 Age_err=[rrsl/(Lambda(Isochron)*(1+Slope))

11070 EHD If

11076 CASE 1 ! a normal (beta-alpha) Pb isochron

11082 CALL Pb_pb_age(Slope, Rge, Errsl, Bge_err, Hi_age, Lo_age)

11088 CASE 2 ! a 207/206 us 204/206 Pb isochron

11094 CALL Pb_pb_age(Inter, Rge, Errint, Bgge_err, Hi_age, Lo_age)

11100 ENO SELECT

11106 !

11112 If Isochron OR Pbph THEN

11118 Print_age:FOR Printer=1 To Prtr

11124 PRINTER IS Pr(Printer)

11130 If Age theN PRINT "HOOEL"; Model;" ISOCHRON AGE =";OROUND(Age,5);

11136 If Rge_err THEN PRINT " +/-";OROUNO(Rge_err,3);

11142 If Rge iHEN PRINT USING "K, $t^{*} ; "$ Ma"

11148 If Pbpb THEN PRINT USING "4K,5(K), I";"(>",Lo_age," (",Hi_age,")"

11154 PRINT USING "l,K";RPT $\$\left(" x^{*}, 80\right)$

11160 NEXI Printer

11166 PRINI USING "3/"

11172 ENO If

11178 ELSE

11184 ! Calculate, printout \& label the concordia intercepts

11190 MAT Cint $=(0)$

11196 GRAPHICS Off

11202 OISP "Calculating concordia intercepts and errors..."

11208 Conc_inters(Slope,Inter, Cint $(*))$

11214 Cint_errs(Model,Slope, Inter,Y_errsl(*),Y_errint(*),Xbar $(*)$, Cint(*))

11220 If Cplot RND forced_inter THEN

11226 If Cint(7)<.01 THEN ! Ruoid too many significant figures here

$11232 \quad \operatorname{Cint}(5)=[\operatorname{int}(2)$

$11238 \quad[\operatorname{int}(6)=[\operatorname{int}(2)$

$11244 \quad[$ int $(7)=0$

11250 END If

11256 If $\operatorname{cint}(8)<.01$ TheN

$11262 \quad \operatorname{Cint}(3)=[\operatorname{int}(1)$

$11268 \quad$ Cint $(4)=[$ int $(1)$

$11274 \quad \operatorname{Cint}(8)=0$

11280 END IF

11286 EHO If

11292 DISP

11298 Print_inters (Cint_r $r(*), \operatorname{Cint}(*), \operatorname{Pr}(\operatorname{Prtr}))$

11304 Label_inters(Cint(*),N_interlabel5, Mswd0)

11310 If Crt PNO N_interlabeis(3 THEN ! Cache results for later plotter-domp

11316 Cache_nsud(N_interlabels)=\#lswdD

11322 FOR I=1 108

11328 Cache_cint $\left(N_{-}\right.$interlabels, I $)=$[int (I)

11334 NEXI I

11340 END If

11346 If Crt THEN CRLL Graph

11352 HAIT 2

11358 END If

11364 Yorkexit:If Crt THEN CALL Alpha

11370 SUbexII

11376 !

11382 Call_help5:CALL Help5

11388 60To Choose_nodel

11394 !

11400 Underflow: ! numeric under/overflow in chi-square prob. calculation 


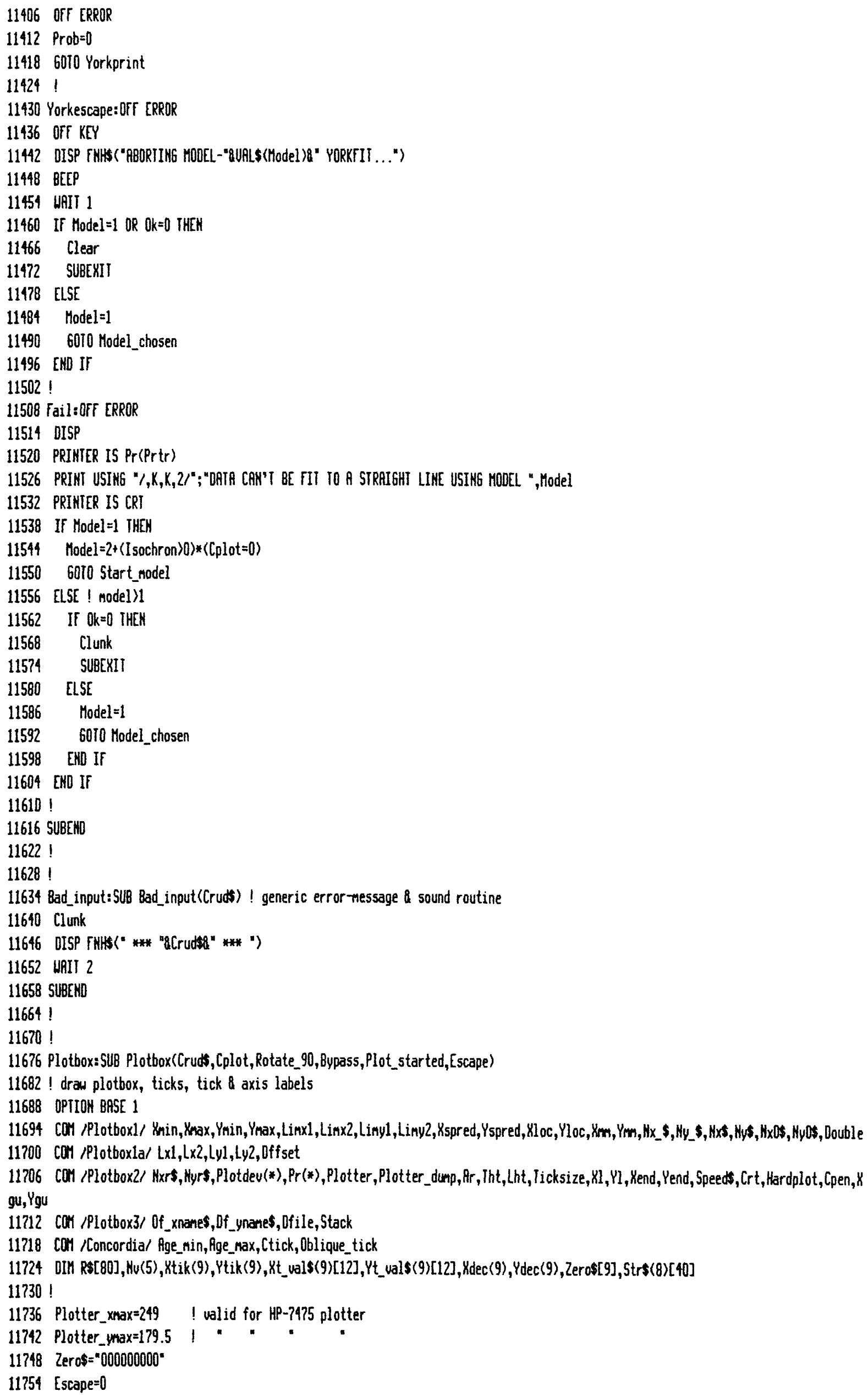




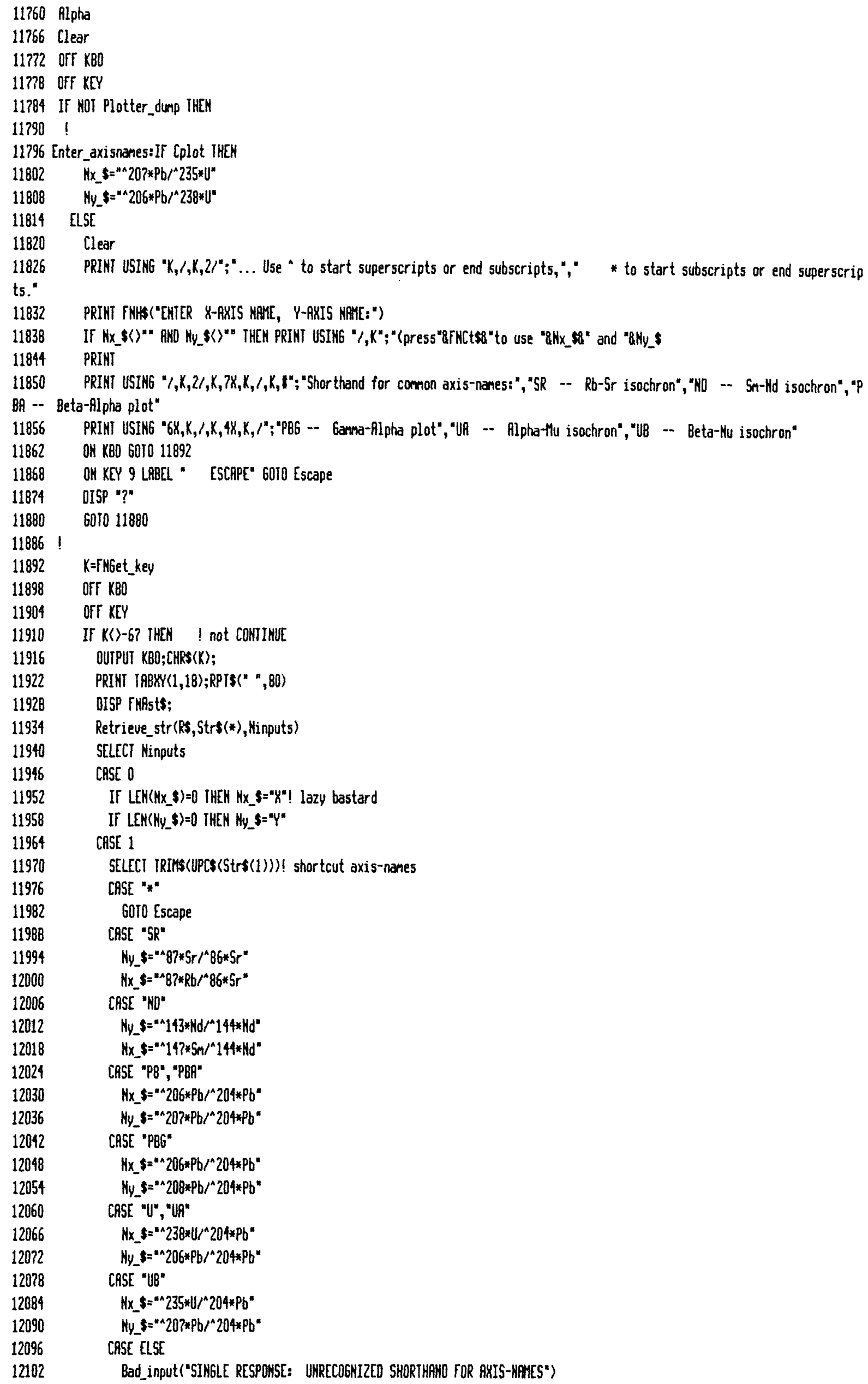




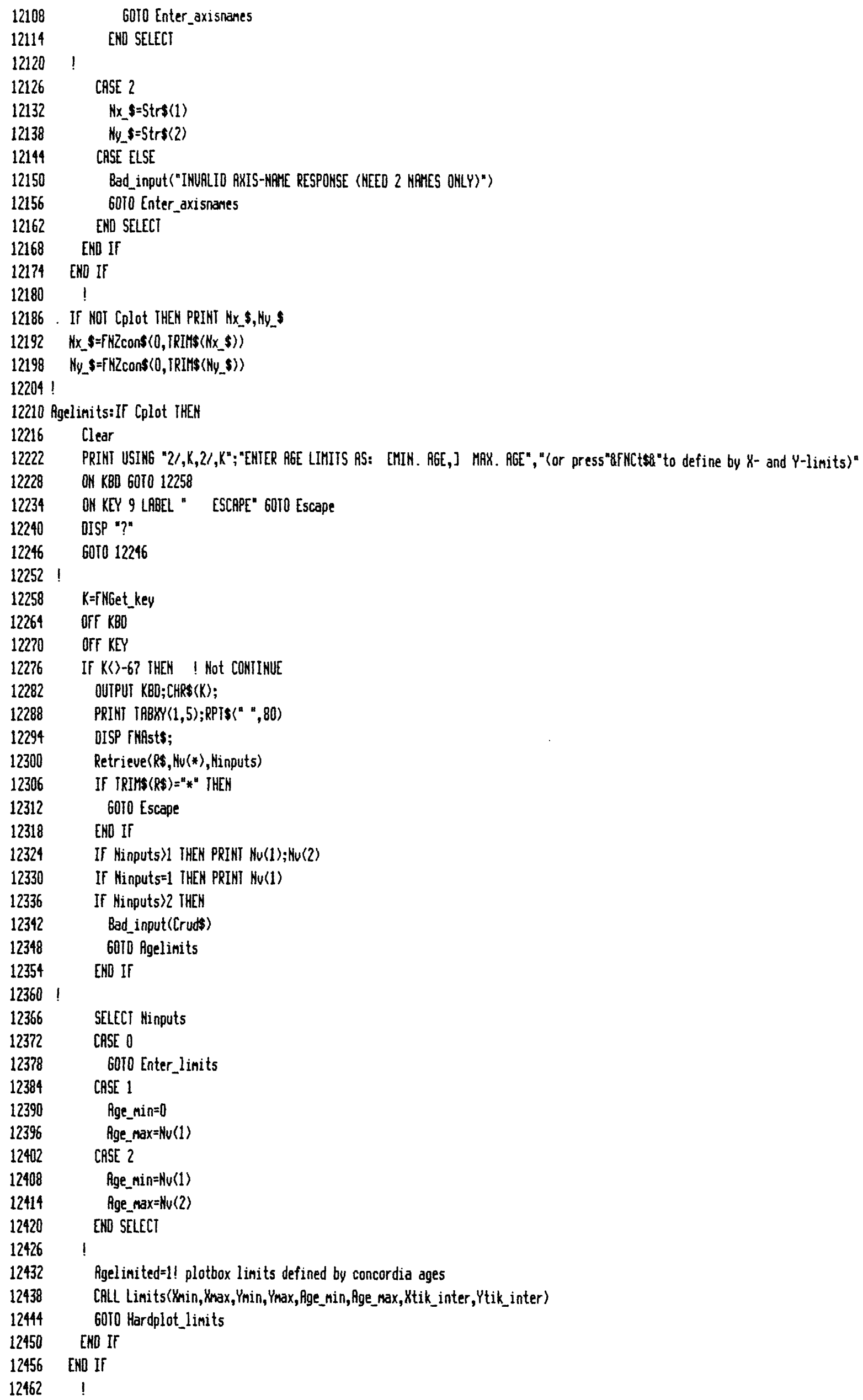


12468 Enter_limits:Clear

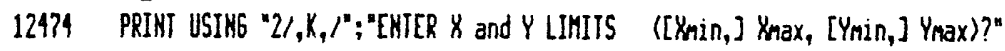

12480 If $\left(Y_{\max }-Y_{\min }\right)$ AND $\left(Y_{\max }-Y_{\min }\right)$ THEN

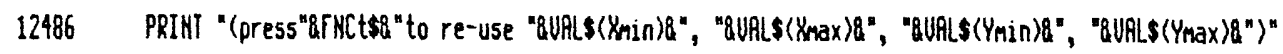

12492 END If

12498 ON KBO 601012528

12504 ON KEY 9 LABEL " ESCAPE" GOTO Escape

12510 DISP "?"

$12516 \quad 607012516$

12522 !

$12528 \quad k=$ NHGet_key

12534 off $\mathrm{KBO}$

12540 OfF $\mathrm{KEY}$

12546 If $K \backslash>-67$ THEN ! Not CONTINUE

12552 OUTPUT KBD; CHRS $(K)$;

12558 PRINT TABXY (1,5);RPTS(" ",80)

12564 DISP FNA5ts;

12570 Retrieve(Rs, Hu(*), Ninputs)

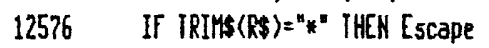

12582 PRIKT R

12588 If Minputs $>>0$ anD Ninputs $>>2$ AND Ninputs $>4$ THEN

12594 CRLL Bad_input(Crud\$)

12600 60T0 Enter_limits

12606 END If

12612 SELECT Ninputs

12618 CASE 2

$12624 \quad x_{\min }=0$

$12630 \quad Y_{\min }=0$

$12636 \quad Y_{\max }=H v(1)$

$12642 \quad Y_{\max }=H u(2)$

12648 CASE 4

$12654 \quad x_{\min }=\mathrm{Nu}(1)$

$12660 \quad Y_{\min }=H u(3)$

$12666 \quad \operatorname{Mmax}_{\max }=4 \mathrm{u}(2)$

$12622 \quad Y_{\max }=N_{v}(4)$

12678 END SELECT

12684 END IF

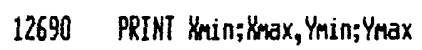

12696 If $X_{\max }=\psi_{\min }$ OR $Y_{\max }=Y_{\min }$ THEN

12702 CALL Bad_input("HAX. LIMIIS MUST BE MORE IHAM MIN. LIMITS")

12708 60T0 Enter_limits

12714 END If

12720 If Cplot THEN

12726 Rige_nin=t1RK(FNO(YMin), FHE (Ynin))

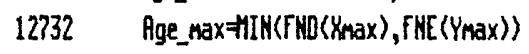

12738 - Agelinited $=0$

12744 END IF

12750 ENO If ! plotter-dunp endif

12756 !

12762 Hardplot_linits:0ff KBD

12768 off $\mathrm{KEY}$

12724 If NOT Plotter_dump IHEN

12780 If Hardplot THEN

$12796 \quad$ Clear

12792 PRINT USING "8/,K,Z/"; "ENTER PLOT-SIZE (3-10)?"

12798 PRINT USING "K, $/$ "; "(Press" 8 FNCte 8 "for default size of 8 "

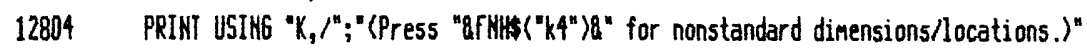

$12810 \mathrm{Hs}_{-} \mathrm{kbd}$ :0N KBO 607012846

12816 ON KEY 9 LABEL " ESCAPE" GOTO Escape

12822 ON KEY 4 LABEL. " NOKSTANDARD" GOTO Nonstd 


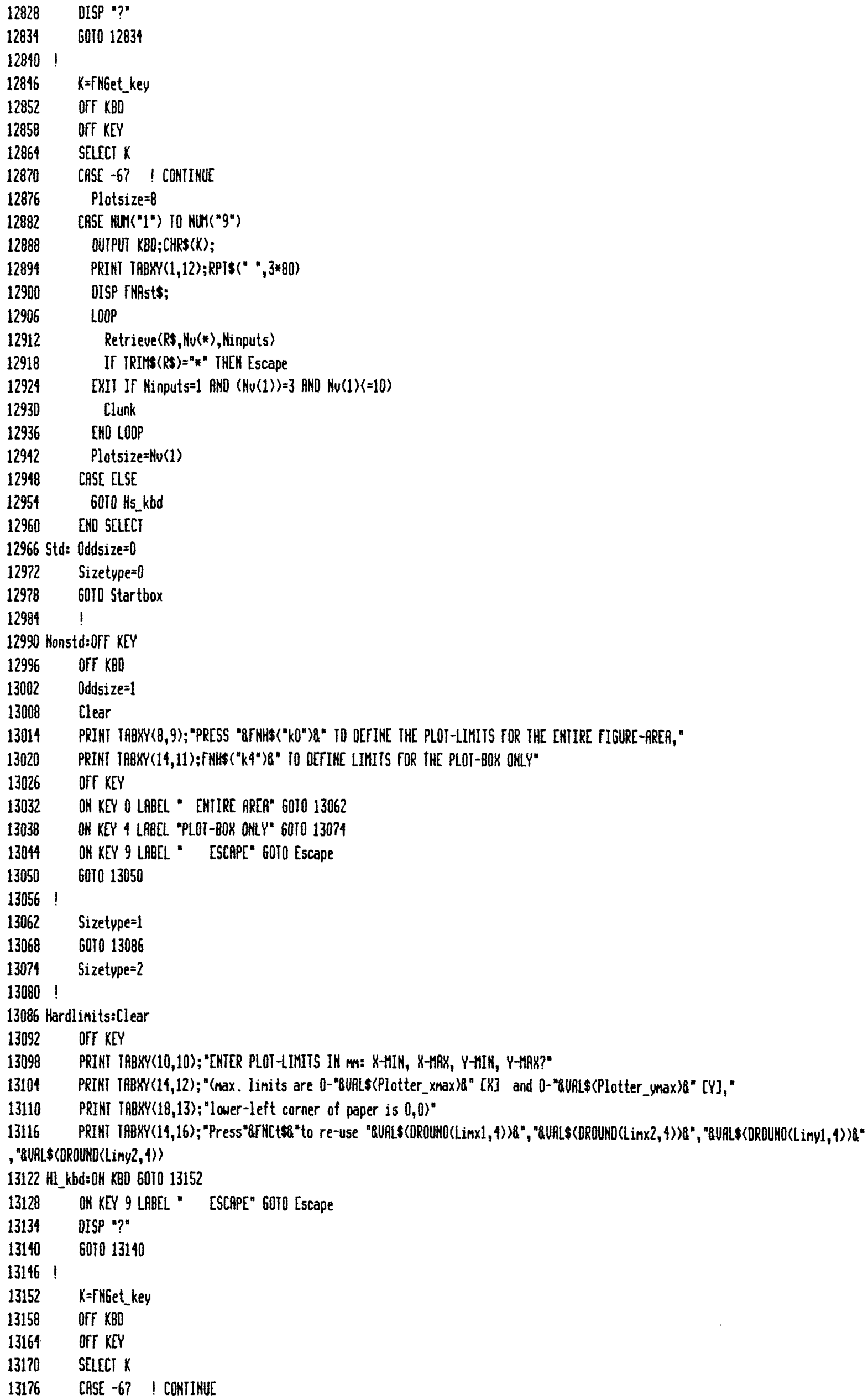


CASE NUM("O") TO NUM("g"), NUH(".")

13188 OUTPUT KBD;CHRS(K);

13194 PRIKT TABXY(1,16);RPIS(" ",80)

13200 DISP FNAsts;

$13206 \quad$ LOOP

13212 INPUT Limx1, Limx2,Liny1,Liny2

13218

13224

13230

13236

13242

13248

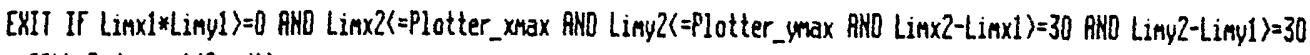
CRLL Badinput(Cruds) END LOOP

CASE ELSE

GOTO HI kbd

ENO SELECT

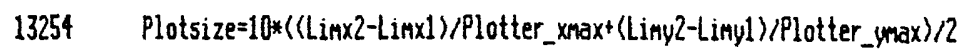

13250 ELSE

13266 Oddsize $=0$

13272 Sizetype $=0$

13278 Plotsize $=\#(1)$

13284 If Plotsize $=10$ THEN Plotsize $=9.9$

13290 ENO If ! hardplot endif

13296 ELSE I Plotter-dump

13302 Oddsize $=0$

13308 Sizetype $=0$

$13314 \quad$ Plotsize $=8$

13320 END IF ! Plotter-dump endif

13326 !

13332 Star tbox:Clear

13338 OFF KEY

13344 OFF KBD

$13350 \mathrm{H} \times \mathrm{H}=\mathrm{HX}_{\mathrm{X}}$

13356 Nys=Ny_s

13362 If Crt THEN

13368 PLOTTER IS 3, "IKTERNAL"

13374 GRAPHICS OK

13380 END IF

13386 !

13392 If NOT Cplot OR NOT Rgelinited THEN ! draw box with ticks

13398 CRLL Tick(Y)

13404 CALL Tick(Ynax-YMin,Ytik_inter)

13410 END IF

13416 !

13422 ! Force lower-bound $X \& Y$ values to have a minimum $I$ of sign. figures.

$13428 \quad I=0$

13434 REPEAT

$13440 \quad I=I+1$

$13446 \quad X=F N D$ e(ABS $\left.\left(X_{n i n}\right), 1\right)$

13452 UNIIL I $>$ OR ABS(Xnin)-X(Xtik_inter

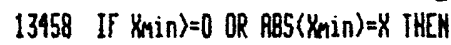

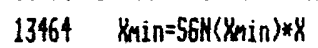

13470 ELSE

$13476 \quad x_{\text {min }}=-x_{-}-x_{\text {tik_inter }}$

13482 ENAD IF

13488 !

$13494 \quad I=0$

13500 REPEAT

$13506 \quad I=I+1$

$13512 \quad Y=F \operatorname{NDe}\left(\operatorname{ABS}\left(Y_{\min }\right), \mathrm{D}\right)$

13518 UNIIL I $/ 7$ OR RBS(Y min $^{2}-Y(Y$ tik_inter

13524 If $\left.Y_{\text {min }}\right)=0$ OR $A B S\left(Y_{\text {Ain }}\right)=Y$ IHEN

$13530 \quad Y_{\min }=566\left(Y_{\text {Min }}\right) * Y$

13536 ELSE 


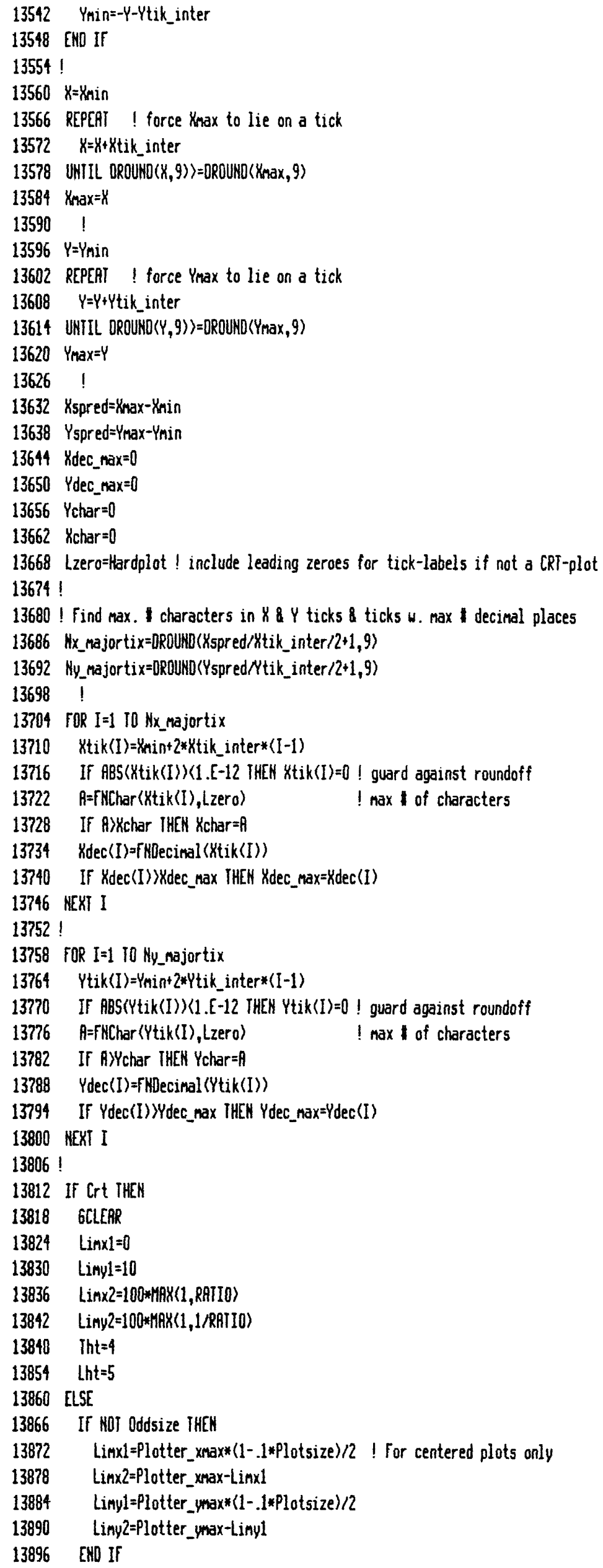


13902 END If

13908 Ar $=.6$ ! Aspect ratio, tick label char. ht.

13914 If Hardplot THEN

13920 Tht $=.45 *$ Plotsize ! character height of tick-labels, in $\mathrm{m}$

$13926 M=.8 *[$ icksize*(1+2.7*(Plotsize(6)/Plotsize)! For plotsizes 66 , sonewhat buffer label-character size.

13932 Tht=1*Tht! Tick-label and axis-label character heights

13938 If Tht 22.0 THEN Tht $=2.0$

13944 Lht $=1.25 *$ Tht

13950 END If

13956 !

13962 Pu=POS (HXS, "A")

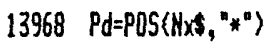

13974 Qu=POS (NyS, "^n)

13980 Qd $=P O S\left(H y S, " * *^{n}\right)$

$13986 P=P U$ OR Pd

$13992 Q=Q Q$ OR Qd

13998 N_xtix=2*Hx_majortix

14004 N_ytix $=2 \times *$ Hy_najortix

$14010 S_{s}=$ (Qu AND Qd RND Qu(Qd)! =1 if Y-axis label has superscripts

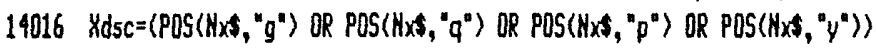

$14022 ! \mathrm{Xdsc}=1$ if the $\mathrm{X}$-axis label contains a character with descenders

14028 Ydsc=(POS(Nys," "q") OR POS(Nys, "q") OR POS(Hus, "p") OR POS(Hys, " "y"))

14034 ! ditto Ydsc for Y-axis label

14040 !

14046 Define_box: !

14052 Yend=Tht/2*FNEven(K_ytix) I6U's, $m$ above top of box

14058 If Crt THEN

14064 Xend=(Ychar*Hr*Iht/2)*FNEven(N_xtix) ! SU's, $m$ to right of K-axis

$14070 \quad K 1=$ Ar $*$ Tht $* Y c h a r+L h t *(17 / 15+4 / 15 * * d s c+5 / 15 * Q) !$ GU's, $m$ to left of $Y$-axis

$14076 Y 1=T h t * 15 / 15+L h t *(1-C r t / 15+4 / 15 *$ Hdsc+4/15*P)! GU's,m below $X$-axis

14082 ELSE! plotter linits

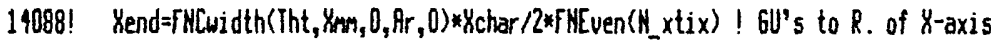

14094 Kend=ychar*Ar*Tht/2*FWEven(N_xtix)! GU's to R. of $Y$-axis

$14100 \quad K 1=$ hr*1.183/.67*Tht*YChar $+L h t *(1+.8+4 / 15 * x d s c+.5 * Q)$ ! 6U's to $L$ of $Y$-axis

$14106 Y 1=T h t * 16 / 15+L h t *(1+.8+4 / 15 * x d s c t .5 * P) \quad$ ! GU's below $X$-axis

14112 END If

14118 !

14124 If Hardplot ANO Sizetype'2 IHEN

14130 Linxl $=$ Linxl+Kl

14136 Linx2=Linx2-Kend

$14142 \quad$ Linyl $=$ Linyl $1+Y 1$

14148 Linyz=Limy2-Yend

14154 END If

14160 !

14166 Rat=RATI0*Crt+(Linx2-Linx1)/(Liny2-Liny1)*Hardpl ot

$14172 \mathrm{Kgu}=100 \times 4$ HAX(1,Rat) ! total GU's of plot $\langle X\rangle$

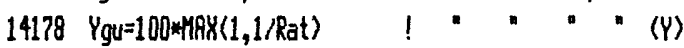

14184! Kgu_mo=(Limx2-Linxl)/Ygu ! m per GU (X), CRT

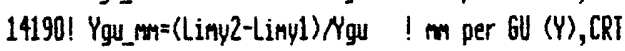

14196 !

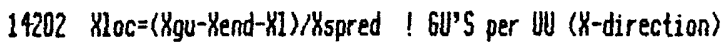

14208 Yloc $=\left(Y_{g u} Y_{\text {end }}-Y 1\right) /$ spred ! GU'S per WU (Y-direction)

14214 If Crt IHEN

$14220 \quad B=1$

14226 ELSE

14232 D=Plotter_ymax/100 I $m$ to bl conversion for 7475 (8.5x11" paper)

14238 END If

$14244 L x|=\operatorname{Lin} x| / 0$

$14250 \quad L \times 2=\operatorname{Lin} \times 2 / 0$

14256 Lyl $=$ Linyl/D 
14262 Ly2=Limy2/0

14268 If Crt IHEN

$14224 \quad X_{m}=((L$ inx2-Limxl-(X1+Xend))*160/133.44)/Xspred ! m/UU $(X)$

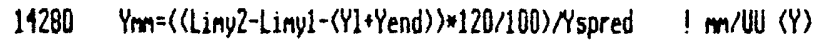

14286 ELSE

$14292 \quad x_{m}=(\operatorname{Lin} \times 2-\operatorname{Linx} 1) / x_{\text {spred }}$

$14298 \quad Y_{m}=(L i n y 2-L i n y 1) / Y_{s p r e d}$

14304 ENO IF

$14310 !$

14316 FOR $D x=0$ Io Double

14322 VIEUPORT $L x 1+0 x * 0 f f$ set, $L 2 \times 2+0 x * 0 f f$ set, $L y 1+0 x * 0 f f$ set, $L y 2+0 x * 0 f f$ set

14328 If Hardplot THEN

14334 UINDON Knin, Yhax, Ymin, Ymax

14340 DUTPUT Plotter;Speeds

14346 If Rotate_90 THEN OUTPUT Plotter; "R090;"

14352 If HOT Rotate_90 THEN OUTPUT Plotter; "RO;"

14358 ELSE

14364 UIKDow Ynin-Yl/Kloc, Ymax+Yend/Yloc, Ymin-YI/Yloc, Yhax+Yend/Yloc

14370 [ND IF

14376 CSIZE Tht, Ar

14382 ! Label every th tick, not every other tick if little room available

14388 xtm $=2 * x+$ tik_inter $* x+m ! m$ between every other tick on $y$-axis

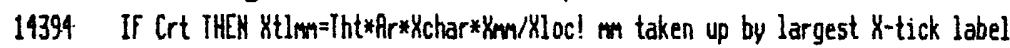

14400 If Hardplot THEN Ktlm=Tht*AF*1.183/.67 ! $m$ taken up by largest $x$-tick label

14406 If $\mathrm{xtlm} / \mathrm{Xtm}$. 5 THEN ! Avoid crouding $\mathrm{X}$-tick labels

$14412 \quad$ Mtik $=2$

14418 ELSE

$14424 \quad$ Mtik=4

14430 END If

14436 !

14412 If HOT Bypass IHEN

14448 Ticklabel (Xt_val\$(*),Ytik(*),Ydec(*),Xdec_max, LzerO,NX_majortix)

14454 Iicklabel(Yt_val\$ $\$(*), Y t i k(*), Y d e c(*), Y$ dec_max, Lzero, Hy_najortix)

14460 END IF

14466 !

14472 Xticksize $=2.5 \mathrm{Nloc}$

14478 Yticksize $=$ Xticksize*\%/m/ $/ \mathrm{H}_{\mathrm{m}}$

14484 If Crt THEN Cpen=1

14490 If HOT Bypass IHEN

14496 PEY Cpen

14582 If OROUKD (Knin, 9) $=0$ THEN Xnin=0 ! roundoff problens?

14508 If DROUND(Ynin, 9) $=0$ THEN Ymin=0 ! roundoff problems?

14514 MOUE Knin, YMin

14520 !

14526 ! draw axes and ticks

14532 Axis(1, Xspred,Xtik_inter, Xticksize,1)

14538 Axis(1,Yspred,Ytik_inter,YYticksize,0)

14544 Axis(-1, Xspred,ytik_inter, Yticksize,1)

14550 Axis $\left(-1, Y_{5 p r e d}, Y t i k_{-}\right.$inter, Yticksize,0)

14556 CLIP OFF

14562 !

14568

If NOT Stack THEN

$14524 \quad$ LORG 6

14580 FOR I=1 TO Hx_majortix STEP Htik/2! Label \&-ticks

14586 MOUE Xtik(I), Ymin

14592 LABECL $X t_{\text {_tals }}$ (I)

14598 HEXT I

14604 END If 
If Crt THEN $B=$ Tht*AR/2/1810c

If Hardplot ThEN $B=$ FNCuidth(Tht, $4 \mathrm{~m}, 0, \mathrm{Br}, 0) / 2$

FOR I 1 I 10 Ny_najortix

14640 ! Slightly offset 1st $y$-tick to avoid overlap w. 1st $x$-tick

14646 If NOT Stack OR I/I IHEN

14652

14658

14664

14670

14676

14682

14688

14694 If Crt THEN HOUE Xnin-B, Ytik(I)*(I=1)*Tht/3/Mloc

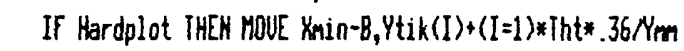
LABEL Yt_vals(I)

END If

NEXI I

ENO If

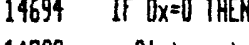

14700 Stripscript(Nus)! convert super/subscript tokens

14706 Stripscript(KXS)

14712 Remove(NxS, Hxrs, LX)! renove super/subscript tokens

14718 Renove(Hys, Hurs, Ly)

14724 ENO IF

14730 !

14736

14742

14748

14754

CSIZE Lht, Ar

CLIP off

LORG 1

If NOT Bypass Then

14960 If NOT Stack THEN

14766 If Crt THEK

14772 MOUE $X_{\text {in }}+Y_{\text {spred }} / 2, Y_{\text {min }}-\left(Y_{1}+L\right.$ Lht $\left.* 4 / 15\right) /$ loc

14778 IMOUE -LEK(XXr\$)/2*LLt:Ar/Kloc,0

14784 ELSE

14790 MOUE $X_{\text {min }}+Y_{5 p r e d} / 2, Y_{\text {min }}-\left(Y_{1}+L\right.$ Lht*9/15)/Mm

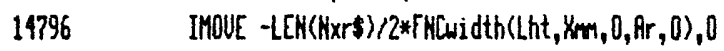

14802 END If

14808 FOR I $=1$ TO LEW $($ HXs)

$14814 \quad S=(N \times \$[I, I]=C H R S(30))-(N \times S[I, I]=C H R S(10))$

14820 If 5 IHEN

14826

14832

14838

14844

14850

14856

14862

14868 !

14874

14880

14886

14892

14898

14904

14910

14916

14922

14928

14934

14940

14946

14952

14958

14964

If Crt THEN IMOUE $0,5 * 5 / 15 *$ Lht $/$ loc

If Hardplot THEN IMOUE $0,5 * .5 *$ Lht $/ \mathrm{mm}$

ELSE

LABEL HXs[I,I];

ENO If

NEXT I

END IF

LOIR 90

If Crt IHEN

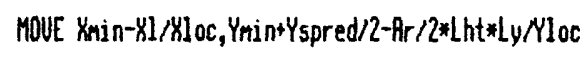

IMOUE Lht*(14/15+5s*5/15)/Kloc, 0

ELSE

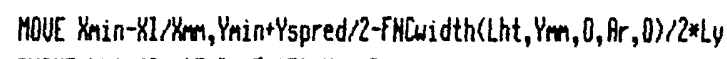

IMOUE $\operatorname{lht} *(21 / 15+5 \% * 5 / 15) / K_{\mathrm{m}}, 0$

END If

FOR I=1 TO LEN(Hys)

$S=($ Hus $5[\mathrm{I}, I]=$ CHRS $(30))-($ Nys $[I, I]=$ CHRS $(10))$

If $S$ THEN

If Crt THEN IMOUE $-5 / 15 * 5 *$ Lht/ $/ 10 \mathrm{loc}, 0$

If Hardplot THEN IMOUE - $-5 \times 5 *$ Lht/ $/ \mathrm{km}, 0$

ELSE

14976 END IF

LABEL Hys[I,I];

END IF

MEXT I 
14988 LDIR 0

14994 NEXT OX

$15000 \mathrm{~N} \times \mathrm{S}=\mathrm{N} \times \mathrm{xr} \boldsymbol{S}$ ! Stripped of " and * characters

15006 Rys=Hyrs

$15012 \mathrm{HXOS}=\mathrm{H} \times \mathrm{x}[1, \mathrm{MIN}(10, \mathrm{LEH}(\mathrm{H} \times \mathrm{s}))]$

15018 HyOs=Hys[1, MIN(10,LEN(Nys))]

15024 If hardplot AND NOT Cplot IHEN PEN O

15030 CLIP Ymin, $Y_{\text {max }}, Y_{\text {min }}, Y_{\max }$

15036 Plot_started=1

15042 SUBEXII

$15048 \quad !$

15054 [scape:[scape 1

15060 SUBEND

15066 !

15072 !

15078 Drauline:SuB Drauline(Slope,IInter, Klines, Solid_line,Line(*)) ! plot the best-fit line

15084 OPTION BRSE 1

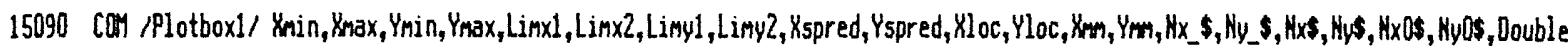

15096 Cot /Plotboxla/ LxI,LX2,Ly1,Ly2,0ffset

15102 COM /Plotbox2/ Hxrs, Hyrs, Plotdeu(*),Pr(*),Plotter,Plotter_dump, Ar, Iht, Lht, Iicksize, Kl, Yl, Kend, Yend, Speed\$, Crt, Hardplot, Cpen, X gu, Ygu

15108 Hlines=Hlinest(Nlines $\{10)$

15114 If Crt THEN

15120 Line(Hlines, 1$)=$ Slope

15126 Line(klines, 2)=Inter

15132 END If

15138 LINE TYPE $1+($ Solid_line $=0) *(4+$ Hlines)

15144 CLIP Ymin, $x_{\text {mint }} .97 * Y_{\text {spred, }} Y_{\text {min, }} Y_{\text {Mint }} .97 * Y_{\text {spred }}$

15150 PEN Cpen

15156 FOR DX=0 To Double

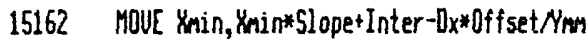

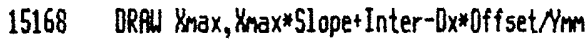

15174 NEXI $D X$

15180 MOUE Xmax, Y $Y_{\max }$

15186 LINE TYPE 1

15192 PEN Crt

15198 CLIP Xmin, $X_{\max }, Y_{\min }, Y_{\max }$

15204 SUBEND

15210 !

$15216 !$

15222 Letter: SU8 Letter (Letter\$(*), Letter (*), Nphrases, Phrase)

15228 APIIOH BRSE 1

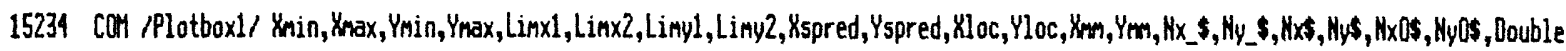

15240 Can /Plotboxla/ LxI, Lx2, Ly1, Ly2, Off set

15246 conl /Plotbox2/ Hxrs, Hyrs, Plotdev(*),Pr(*),Plotter,Plotter_dump, Ar, Tht, Lht, Ticksize, X1,Y1,Yend, Yend, Speeds, Crt, Hardplot, Cpen, X giu, Ygu

15252 DIM RS[32], Hu(4), P5[80], Pn (7,3)

15258 마 $1,10,-2,7,10,-1,4,13,-2,4,7,-1,1,4,-2,7,4,-1,9,1,-2$

15264 REAO Pm(*) ! plus-or-minus symbol

$15270 \quad !$

15276 first_letter $=1$

15282 Clear

15288 GRPPHICS OFF

15294 CLIP OFF

15300 OfF $\mathrm{KEY}$

15306 off KBD

15312 !

15318 If HOT Plotter_dump THEN

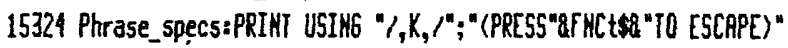




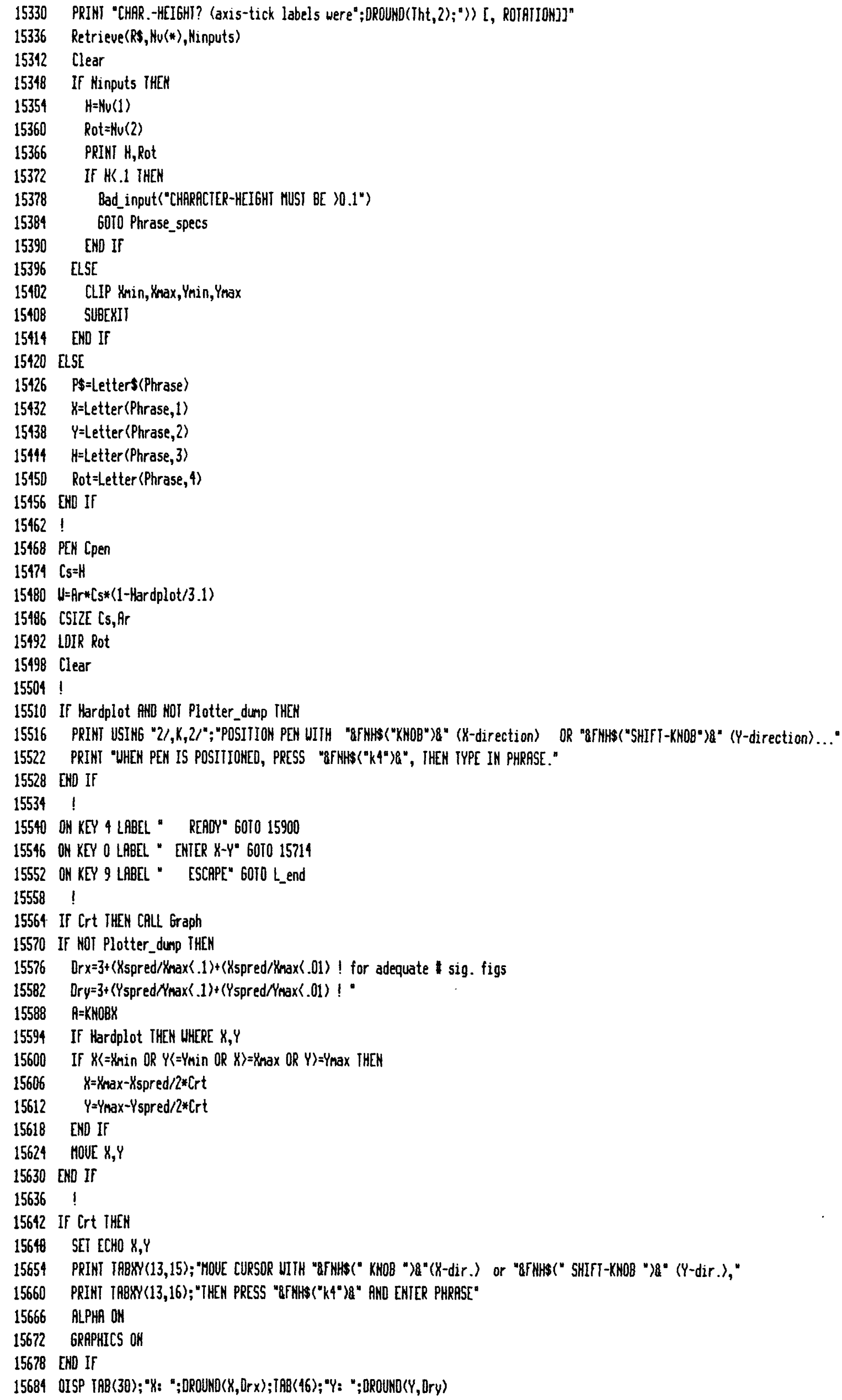


15690 If Plotter_dump THEN 15936

15696 ON KNOB .1 GOSUB 15798

15702601015702

$15708 \quad !$

$15714 ! x-y$ locating

15720 LOOP

15726 DISP "ENTER COOROIMATES $(X, Y)$ OF LOUER-LEFI CORNER OF PHRASE";

15732 Retrieve (Rs, Nu(*), Ninputs)

15738 EXIT If Ninputs $>1$

15744 Clunk

15750601015900

15756 ENO LOOP

$15762 x=H v(1)$

$15768 Y=H u(2)$

15774 PRIHT $X, Y$

15780 PEN Cpen

15786607015900

15792

15798 STATUS 2,10;0ir

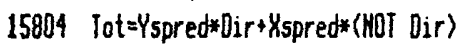

15810 Del ta=KNOBX/600*Tot/(1+Hardplot)

15816 If Dir $=0$ THEN $X=X+$ Delta

15822 If Dir THEN $Y=\psi+$ Delta

15828 If Crt IHEW

15834 If $Y>\rangle_{\text {max }}$ THEC $X=Y_{\text {nin }}$

15840 If YYYmax THEM $Y=Y_{\text {min }}$

15846 If $K\left(X_{\text {min }}\right.$ THEN $Y=Y_{\max }$

15852 If $Y$ Y Y min THEN Y $=Y_{\text {max }}$

15858 SET ECHO X,Y

15864 ELSE

15870 MOUE $X, Y$

15876 ENO IF

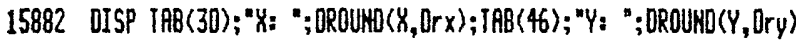

15888 RETURH

$15894 \quad !$

15900 OFF KBD

15906 OFF KHOB

15912 Clear

15918 MOUE $X, Y$

15924 If Crt THEN SET ECHO $2 * *_{\max }, 2 * Y_{\max }$

15930 !

15936 LORG 1

15942 If Crt THEN CALL Alpha

15948 If NOT Plotter_dump IHEN

15954 LIRPUT "PHRASE TO BE ORATIEO?", PS

15960 END If

15966 Clear

15972 PRINT Ps

15978 Stripscript(P\$)

$15984 P S=F M Z \operatorname{con} S(0, P \$)$

15990 L=LEN(P\$)

15996 !

16002 Charwidth=FNCuidth(Cs, $y_{m,}, X_{10 c}, A r,[r t)$

16008 Charheight $F F$ Mheight $\left(C_{5}, Y Y_{0 c}, Y_{m m},[r t)\right.$

16014 !

16020 FaR $D x=0$ io Double

16026 If OX IHEN

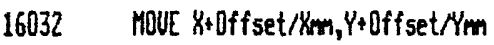

16038 ELSE

16044 If Crt THEN 


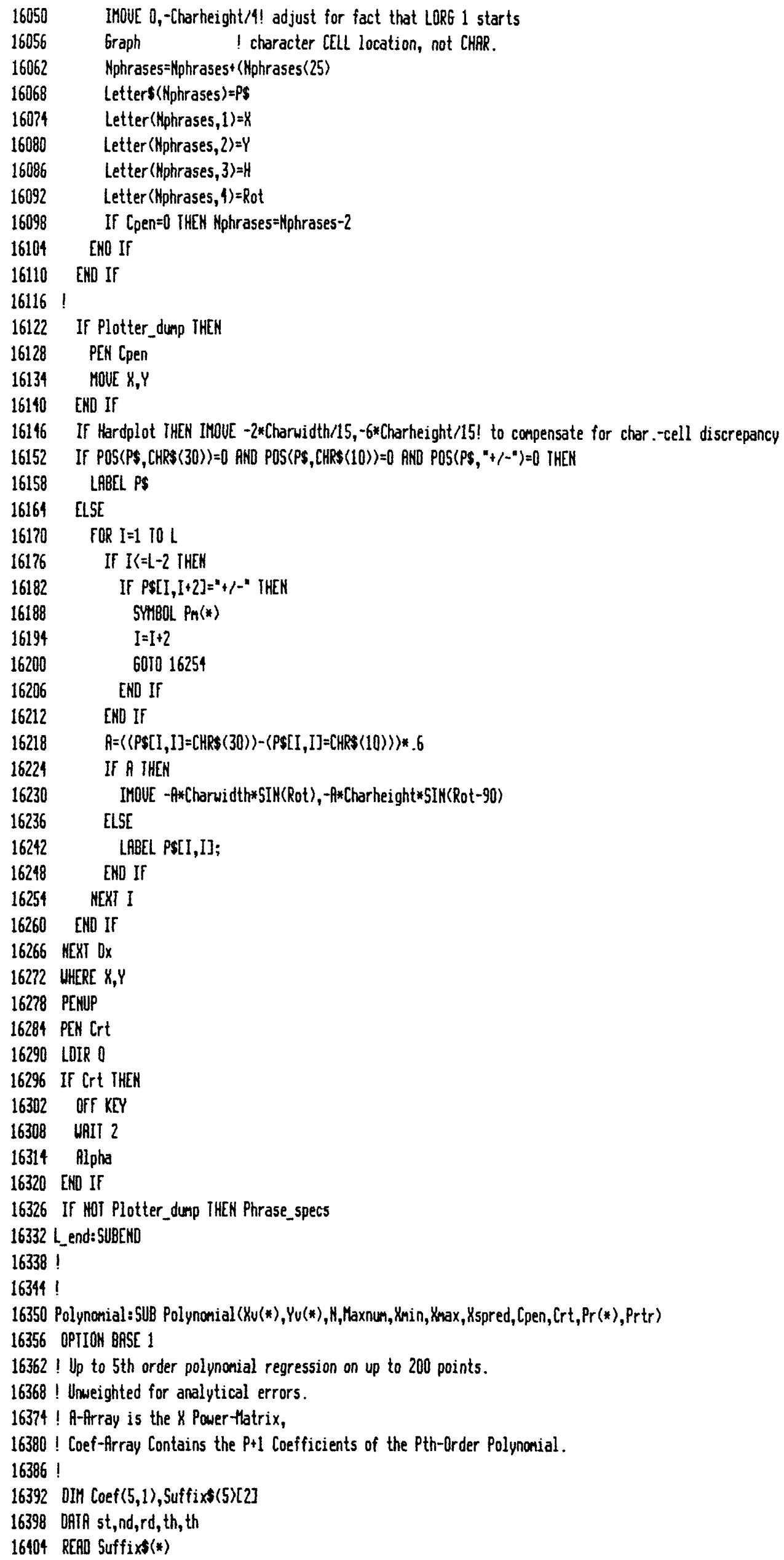


16410 Clear

16416 DFF KEY

16422 OFF KBD

16428 If N3200 THEN

16434 Bad_input("**** CAN'T FII MORE THRM 200 POIHTS *****")

16440 SUBEXIT

16446 ENO IF

16452 Max_order $=\#-1$

16458 If Max_order $>4$ THEW Max_order $=4$

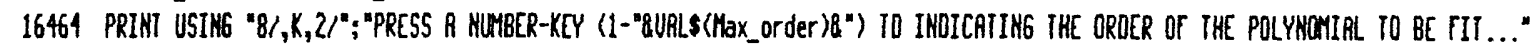

16470 PRIHT "(Press"afNCts8"to escape)"

16476 ON KBO 601016488

16482 GOTD 16482

$16488 \mathrm{k}=$ FHGet_key

$16494 \quad P=K-48$

16500 BEEP

16506 OFF KBD

16512 Clear

16518 If $k=-67$ JHEN SUBEXII ! CONIINUE key pressed

16524 If $P \angle D$ OR P>9 THEN 16410 ! illegal key

16530 If P(1 OR PYlax_order THEN

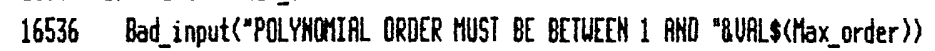

16542607016410

16548 END If

16554 !

16560 Polycalc $\left(Y_{u}(*), Y u(*), \operatorname{Coef}(*), P, H\right)$

16566 !

16572 POR $J=1$ TO Prtr

16578 PRINTER IS $\operatorname{Pr}(J)$

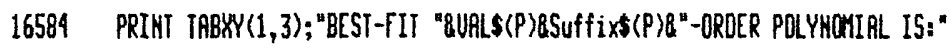

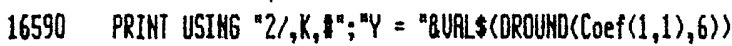

16596 FOR I $=1$ IO P

16602 If Coef $(I+1,1)<0$ THEN PRINT " -";

16608 If Coef $(I+1,1)\rangle=0$ THEN PRINT " +";

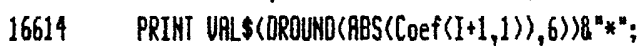

16620 If I=1 THEN PRINT " $x$ ";

16626 If I 11 THEN PRINT "Y^*QURLS(I);

16632 HEXT I

16638 PRINT

16644 NEXT J

16650 OISP

16656 PRINTER IS CRT

16662 PEN Cpen

16668 If Crt THEN CRLL Graph

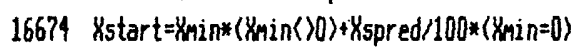

16680 FOR I $=$ Xstart TO Xnax STEP (Xspred)/100

$16686 \quad Y=0$

16692 If I=O THEN GOTO 16752

16698 FOR $J=1$ TO $P+1$

$16704 \quad \psi=\psi+\operatorname{Coef}(\mathrm{J}, 1) \times\left[\left[^{\wedge}(\mathrm{J}-1)\right.\right.$

16710 HEXT J

16716 If I $>>$ Sstart THEN

16722 OH ERROR GOTO 16752

16728 ORPU I,Y

16734 ELSE

16740 MOUE $I, Y$

16746 END If

16752 MEXI I

16758 PEN 0

16764 Alpha 
16770 DISP "PRESS "8FNCtSB" IO CONIINUE"

16776 PRILSE

16782 SUBEXIT

16788 !

16794 RETURH

16800 SUBENO

$16806 !$

$16812 !$

$16 B 18$ Expo_fit:SUB Expo_fit(N, $\left.X_{u}(*), Y_{u}(*), X_{\text {min }}, X_{\text {max }}, Y_{\text {min }}, Y_{\text {max }}, C_{\text {Crt }}, C_{p e n}, \operatorname{Pr}(*), \operatorname{Prtr}\right)$

16824 OPTIOH BASE 1

16830 OFF KEY

16836 Clear

$16842 \quad Z=N$

$16848 \mathrm{BO}=1$

16854 OH [RROR GOSUB Hext_maxpt

$16860 B D=L O G(Y u\langle Z) / A O) / K u(Z)$

16866 OFF ERROR

16872 LOOP

16878 OATR $0,0,0,0,0,0$

16884 RESTORE 16878

16890 REAO $\mathrm{FO}, \mathrm{A1}, \mathrm{B1}, \mathrm{B2}, \mathrm{Cl}, \mathrm{C2}$

16896 FOR $I=110 \mathrm{~K}$

$16902 \quad F O=Y u(I)-A D *[X P(B D * X U(I))$

$16908 \quad A 1=91+Y u(I) \wedge 2$

$16914 \quad B 2=B 2+\left(X_{U}(I) * Y_{U}(I)\right)^{\wedge} 2$

$16920 \quad B 1=B 1+X_{U}(I) * Y_{U}(I)^{\wedge} 2$

$16926 \quad C 1=C 1-Y_{v}(I) * F 0$

$16932 \quad\left[2=\left[2-X_{U}(I) * Y_{u}(I) * 50\right.\right.$

16938 NEXT I

16944 Count $=1+$ Count

16950 If Count>50 THEH

16956 Cant_fit

16962 SUBEXII

16968 END If

16974 ON ERROR G0T0 16956

16980 Al $=$ A1 $/$ /AO^2

$16986 \quad B 1=B 1 / \mathrm{AO}$

$16992 \quad C_{1}=C_{1} / \mathrm{AO}$

$16998 \quad R 2=B 1$

$17004 !$

$17010 \quad A=(C 1-B 1 * C 2 / B 2) /(A 1-A 2 * B 1 / B 2)$

$17016 \quad B=(C 2-A 2 * A) / B 2$

$17022 \quad A=A D-A$

$17028 \quad B=80-8$

17034 Aa $=$ OROUND(A,5)

$17040 \quad B b=$ DROUND(B,5)

17046 DISP 月a; 7 月8(20);Bb

17052 EXIT If Aa=OROUNDCBO,5) OR Bb=DROUHB(BO,5)

$17058 \quad$ BD $=$ 月

$17064 \quad B O=8$

12070 ENO LOOP

17076 !

17082 OFF ERROR

17088 FOR I=1 IO Prtr

17094 PRINTER IS Pr(I)

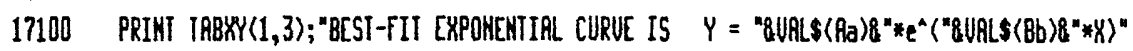

17106 NEXT I

17112 PRINTER IS CRT

17118 PEN Cpen

17124 IF Crt THEN GRPPHICS ON 
17130 FOR $X=X_{\min }$ 10 $X_{\max }-\left(X_{\max }-X_{\min }\right) / 70$ STEP $\left(X_{\max }-X_{\min }\right) / 70$

$17136 Y=$ Y A*EXP(B*X)

17142 If $X) Y_{\text {min }}$ RND Y) Y $Y_{\text {min }}$ AND Y Y Y max THEN

17148 DRRAL $X, Y$

17154 ELISE

17160 MOUE $X, Y$

17166 ENO If

17172 NEXT 8

17178 PEN 0

17189 GRAPHICS OFF

17190 OISP "PRESS "BINCtSL" TO CONTINUE"

17196 PAUSE

17202 SUBEXIT

$17208 !$

17214 Next_naxpt:z=2-1

17220 If 213 IHEN

17226 Cant_fit

17232 SUBEXII

17238 END IF

17244 RETURN

17250 SUBENO

17256 !

$17262 !$

17268 Logar_fit:SUB Logar_fit (Yu(*),Yu(*), H, Xmin, Xmax, Ymin, Ymax, Crt, Cpen,Pr(*),Prtr)

17274 OPIION BRSE 1

17280 OFf KEY

17286 Clear

17292 !

$17298 \quad 2=\mathrm{N}$

17304 AO $=1$

17310 ON ERROR GOSUB Hext_naxpt

$17316 \mathrm{BO}=\left[\mathrm{XP}\left(\mathrm{Y}_{\mathrm{U}}(2) / \mathrm{Hu}(2)\right)\right.$

17322 ON ERROR 6010 Bad_logfit

17328 LOOP

17334 DRTR $0,0,0,0,0$

17340 RESTORE 17334

17346 READ FO, $\mathrm{A1}, \mathrm{B1}, \mathrm{Cl}, \mathrm{C2}$

17352 FOR I $=1$ TO N

17358 If $\mathrm{Ru}(\mathrm{I})<=0$ THEN

17364 Cant_fit

17370 SUBEXII

17376 END IF

$17382 \quad F 0=Y U(I)-R 0 * 106(B D * 8 U(I))$

$17388 \quad A 1=\beta 1+Y_{U}(I)^{\wedge} 2$

$17394 \quad B 1=81+Y u(I)$

$17400 \quad\left[1=C 1-Y_{U}(1) * 50\right.$

$17406 \quad[2=[2-50$

17412 NEXI I

17418 Count $=1+$ Count

17424 If Count $>50$ THEN

17430 Cont_fit

17436 SUBEXII

17442 END IF

17448 ON ERROR GOTO 17430

17454 Al= $11 /$ 月0^?

$17460 B 2=H *(\mathrm{AO} / \mathrm{BO})^{\wedge} 2$

$17466 \quad \mathrm{A2}=\mathrm{B1} / \mathrm{BO}$

$17472 \quad B 1=81 / 80$

$17478 \quad C 1=C 1 / \mathrm{BO}$

$17484 \quad \mathrm{C} 2=C 2 * \mathrm{AO} / \mathrm{BO}$ 


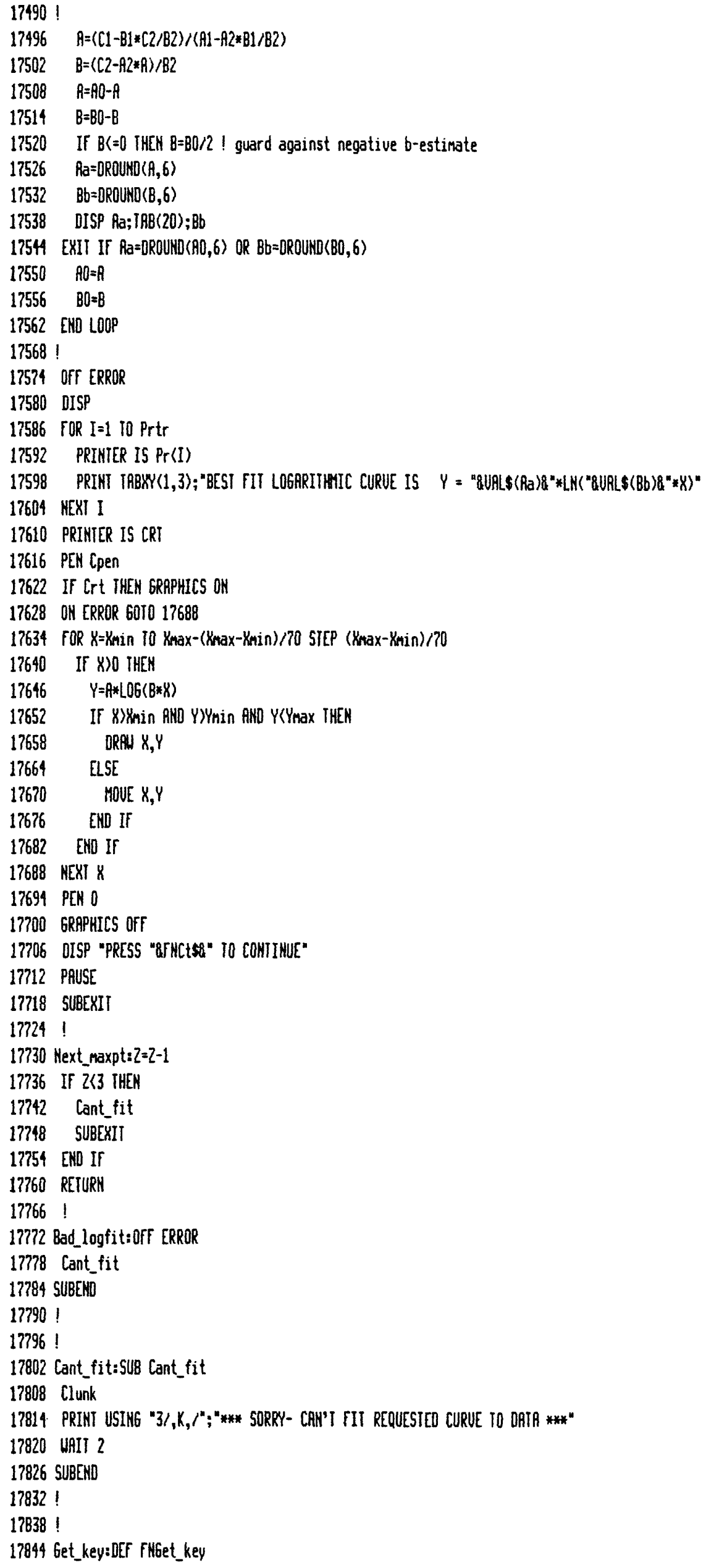


17880 CASE 1

17886 Keycode=Nun(Keys)

17892 CASE 2

17898 Keycodes-Kinc(Keys[2,2])

17904 CASE 3

17910 Keycode=-Nint(Keys[3,3])-128

17916 ENO SELLCT

17922 If Keycode $=-77$ THEN CALL Alpha

17928 If Keycode $=79$ THEN DUAP RLPHR

17934 If Keycodes -76 THEN CALL Graph

17940 RETURK Keycode

$17946 \quad$

17952 Error:RETURN 0

17958 FNENB

$17964 !$

17970 !

17976 Stripscript:SUB Stripscript(S\$) ! change * to subscript token, CIL-* to *

17982 OIM $[\$[80]$

17988 TOR P=1 TO LEN(S\$)

$17994 \quad H=$ HUHKSSEP, P])

18000 SELECT N

18006 CASE $94 !^{\wedge}$

18012 TS[P, P]=CHRS(30) ! CTRL *

18018 CASE 42 !*

18024 TS[P,P]=CHPS (10) ! CIRL *

18030 CRSE 30 ! CIRL *

$18036 \quad T S[P, P]==^{\mathrm{nAn}}$

18042 CASE 10 ! CIRL *

$18048 \quad[\leqslant[P, P]=" * "$

18054 CASE ELSE

$18060 \quad[S[P, P]=S \$[P, P]$

18066 END SELECT

18072 MEXT P

$18078 \quad 5 \$=5 s$

18084 If $5 \$="$ THEN $5 \$="$

18090 SUBEND

18096 !

18102 !

18108 Even: BEF FMEven(K) I deternine whether $N$ is an even integer

18114 If ABS(K/2-INT (N/2)) (1.E-10*N THEN

18120 RETURY 1

18126 ELSE

18132 RETURN 0

18138 ENB IF

18144 FNENB

18150 !

18156 !

18162 Help1: SuB Help1(Cplot) ! helpscreen for initial ("Begin:") display

18168 OPTIOK BASE 1

18174 OIM $5 \$(18)[160]$

18180 ORTA 0: PRINTER OUTPUT Use the external printer as the output device (toggles with CRT OUTPUI). $s$

18186 DATR O: CRT OUIPUT Use the CRT as the printer (toggles with PRIHTCR OUIPUT).\$

18192 DATA 1: PLOTTER PLOT

18198 OATA 1: CRT PLOT Use the external plotter as the plotting device (toggles with CRI PLOT).S

18204 OAIR 2: CHOOSE PEH Use the CRT as the plotting device (toggles with PLOTIER PLOT). (Plotter-plot only) Select a pen of the external plotter.5 
18210 DATR 3: STRCKED PLOT (Plotter plot only) Suppress the X-axis label and tick-labels so that it can be stacked on top of a nother plot that

18216 DATA has the sane $x$-axis and $X$-axis linits.\$4: HELP Invoke this HELP screen.S

18222 DATR"5: ADJ. LABELSIZZ (Plotter-plot only) Expand or contract the relative size of the axis- and tick-labels, as well as al pha-character plotting"

18228 DRTR symbols.S6: ROIATE PLOT (Plotter-plot only) Rotate the plot 90 degrees (toggles uith URROTATEO PLOT). $s$

18234 ORTR 6: UNROTATED PLDT (Plotter-plot only) Use the normal (unrotated) orientation of the plot (toggles with ROTATE PLOT).S

18240 DRTR 7: PEN SPEEO (Plotter-plot only) Select a pen-speed for the external plotter.s

18246 DATR 8: GET OATAFILE Transfer data from a VISICALC file into memory.

18252 DATA SCTRL- $C$ Toggle from Isochron plotter to Concordia Plotter.\$CTRL-I Toggle from Concordia Plotte

$r$ to Isochron Plotter.

18258 DATA"3: DASH CIICK (Concordia plots only) Use a dash-symbol for the concordia ticks, oriented (uith its labels) normal

to the"

18264 DATR" concordia curve (toggles with CIRCLE CIICK).S3: CIRCLE CIICK (Concordia-plots only) Use a small circle for the conco rdia ticks,"

18270 DRTA"with tick-labels oriented horizontal (toggles with DASH CIICK)."

18276 DRTA "\$9: STRRT Enter the axis-names and linits, then start drawing the plot (for a plotter-plot, if you want"

18282 DATR "to bypass the drawing of the plot-box and labels, press CTRL-k9 instead)."

18288 READ $55(*)$

18294 CALL Helpscreen(S\$(*),21)

18300 SUBEND

18306 !

18312 !

18318 Help2:SUB Help2(Cplot)

18324 OPIIOK BASE 1

18330 DIM $5 \$(17)[160]$

18336 DFTh 1: ERROR ELLIPSE Use an open (unshifted) or solid (shifted) error-ellipse as the plotting symbol s

18342 DATA 3: POLYGON

have any number of sides

Use an open (unshifted) or solid (shifted) regular polygon as the plotting symbol. The polygon can

18348 0ATh"and an arbitrary orientation. Star-shaped polygons are also pernitted.54: OpIIONS Select error-input as percen $t$ or absolute,"

18354 OATA"1-signa or 2-signa; do a polynomial, linear, or log fit to data (ISOPLOT only); store the CRI plot on a disk...5"

18366 ORTA 5: 50LIO ISOCHRON (Plotter-plot only) Use solid rather than dashed

18372 ORTR 5: PRINT PLOT (CRI-plot only) Dump the CRT plot to the printer.55: 50LIO ISOCHRON (Plotter-plot only) Use solid rather than dashed

18378 DATh lines to draw isochrons or chords56: ERRSE PEN? (CRT-plot only) toggle between uhite (normal)

18384 OATR and erasing pen.\$8: 6ET DATAFILE Transfer data from a UISICALC file into menory.S

18390 OATR 6: CHOOSE PEN (Plotter plot ony) Select a different pen fron the external plotter.S

18396 0月TA 7: YORKFII Do a Yorkfit (2-error linear regression) to the plotted data (nust have at least 2 points).s

18402 OATA 9: NEL PLOT Start a completely new plot.s

18414 ORTA CTRL-L

18420 DATR CTRL-D

Draft a phrase anywhere on the plot.SCTRL-P

Dump the CRT plot to the external plotter. $\$ *+0 \% \ldots . .$.

Dunp the CRI plot to the printer.s

Use any keyboard-synbol as a plotting

-synbol.s

18426 OATR CTRL-E

Oraw the 95\%-confidence limit error-envelope about the last Yorkfit line. $\$$

18432 OATH CTRL F:

(Concordia-plotter only) Force the next-invoked Yorkfit through an arbitrary age on the concordia-cu

rue.s

18438 OATH O: ERROR BOX

Use an open (unshifted) or solid (shifted) error-box as the plotting symbol.s

18444 DATR 2: ERROR CROSS

Use an error-cross as the plotting synbol.s

$18450 !$

18456 If Cplot THEN REDIM 5\$(15)

18462 REAO $5 \$(*)$

18468 CALL Helpscreen(\$\$(*),21)

18474 SUBENO

$18480 !$

$18486 !$

18492 Help3:SUB Help3

18498 OPIIOK BASE 1

18504 OIM $5 \$(7)[160]$

18510 DRTA 1: ABS ERRORS

PERCENT( rather than 
18516 OATA their RBSOLUTE values).S3: 1-SIG ERRORS Input errors at the 1-SIGHA level rather than the 2-SIGHA level.S4: 2-SI6 E

RRORS Input errors

18522 DAIR at the 2-SIGYA level rather than the 1-SIGAR level.s6: EXPO III fit an exponential curve

18528 OATh to the data of the forn $Y=a *{ }^{\wedge}(B * X) . S 7$ : LOG FII Fit a logarithmic curve to the data of the forn $Y=a * L N(b * X) . S$

18534 OAT 8: POLYII Fit a polynowial curve to the data.s

18540 DRTR D: LORD PLOT PUt a CRT plot stored on a disk onto the CRT .55: STORE PLOT (CRT-plot only) Store the current

CRI graphics on a disk.S

18546 DRTR 9: [SCAPC Return to the calling screen.

18552 READ Ss (*)

18558 CRLL Helpscreen(S5(*),21)

18564 SUBEND

$18570 !$

18576 !

18582 Help6: SUB Help6

$1858 B$ OPIIOH BRSE 1

18594 OIM $5 \$(5)[160]$

18600 DATR 1: PRINT PLOT Bump the CRI plot to the printer \$9: NEW PLOT Abandon this plot and start a new one.\$3: ROD POINTS Add

18612 0月Th more data-points to the plot and pool then with the previously-plotted points for any line- or curve-fitting.\$4: CONIIM UE Return

18618 OATh "to the 'Select Plotting-Symbol' screen, and don't pool any additional data-points with previously-input points for line - or curve-fitting."

18624 DATR "S5: ERROR ENUELOPE Oraw the 95\%-confidence linit error-envelope about the Yorkfit line."

18630 DATR "\$8: DELIEIC poINTS Delete a point fron the next line- or curve-fit."

18636 REAO $55(*)$

18642 CALL Helpscreen $(\$ \$(*), 21)$

18648 SUBEND

18654 !

$18660 !$

18666 Help5: SUB Help5

18672 OPIION BRSE 1

18678 OIM S\$ (5) [160]

18684 DRTA "1: MODEL-1 Choose the MODEL-1 Yorkfit as the best-fit line to the data (assumes that analytical error alone is the cause of the"

18690 ORTh "observed scatter, \& so weights the points according to their analytical errors) .5 "

18696 DRIR "2: MODEL-2 Choose the MODEL-2 Yorkfit as the best-fit line to the data lassunes nothing about the cause of the scatter, and weights"

18702 OATR "each point equally).\$3: MODEL-3 Choose the MODEL-3 Yorkfit as the best-fit line to the data (assumes that the points scatter because"

18708 OATh "of a conbination of analytical error and a normally-distributed variation in the initial-ratios):

$18714 \operatorname{RERB} 55(*)$

18720 CALL Helpscreen $(\$ s(*), 21)$

18726 SUBEND

$18732 !$

$18738 !$

18794 Help4:SUB Help4(Cplot)

18750 OPIION BRSE 1

18756 DIM $5 \$(8)[160]$

18762 0RTh 2: YORKFIT Do a York-type linear regression on the data-points and calculate isochron ages if appropriate.\$3: ADO POIHTS DO

18768 OATR include the last-plotted data-points with subsequently-input points for any line- or curve-fitting.S4: NEW POINTS

DON'I include the

18774 DATh last-plotted points with subsequently-input points for any line- or curve-fitting.s

18780 OATA 8: OELEIE POINT Delete a point for the next line- or curve-fit.\$9: NEW PLOT

Abandon this plot and start a co

ipletely new one.s

18786 ORTR CTRL L:

18787 OATA CIRL E:

Draft a phrase anwwhere on the plot.s

18792 OATA "SSCTRL F:

Oraw the 95\%-confidence linit error-envelope about the last Yorkfit line.

e concordia- intercepts"

(Concordia-plotter only) Do a York-type linear regression on the data points, forcing one of th

18798 0月Th through an arbitrary age on the concordia curve. Calculate the intercepts and errors. 
18804 If MOT Cplot THEN REOIM S\$(6)

18810 RERD SS $(*)$

18816 CfLL Helpscreen(S\$(*),21)

18822 SUBEND

18828 !

18834 !

18840 Pb_pb_age:SUB Pb_pb_age(R76, Rge, OPIIONFL R76_err, Rge_err, Hi_age, Lo_age)

18846 ! calculate $\mathrm{Pb}-207 / 206$ age and (optional) error

18852 OPIION BASE 1

18858 OIM Pb_pb_age(3)

18864 U238_235=137.88

18870 Lambda $238=1.55125[-4$

18876 Lambda235 $=9.8485 E-4$

18882 Age $=0$

18888 If NPAR $=6$ THEN Calc_errs $=1$

18894 MAT Pb_pb_age $=(0)$

$18900 \quad$ !

18906 FOR I=2-Calc_errs TO 2+Calc_errs

$18912 \quad R=R 76$

18918 If Caic_errs THEN

$18924 \quad R=R+(I-2) * R ? 6_{\text {_err }}$

18930 END IF

18936 IF $R>.0156$ RND R $<1.9$ THEN

18942 Trial_t $\left.\left.t=4500 *\left(S 6 H\left(-(1 / 2)+(R) L a n b d a 235 /\left(L a n b d a 238 * U 238 \_235\right)\right)\right)+(R) .7\right)\right)$

$18948 \quad$

18954 Change=1

18960 REPERT

$18966 \quad F=$ Lanbda238*EXP(Irial_t*(Lambda238-Lanbda235))/Lanbda235

18972 I=L06(1+(EXP(Trial_t*Lanbda238)-1-f*(EXP(Irial_t*Lanbda235)-1))/(1/(U238_235*R)-F ))/(anbda235

18978 Change=ABS(T-Irial_t $t)$

$18984 \quad$ Trial_t $t=T$

18990 UKTIL Changel.01

18996 Pb_pb_age $(I)=I$

19002 END IF

19008 NEXI I

19014 Age $=P p_{\text {_p pb_age }}(2)$

19020 If NPRR $=6$ THEN

19026 Hi_age=OROUKO(Pb_pb_age(3),3)

19032 Lo_age=DROUNO(Pb_pb_age (1),3)

19038 If Hi_age AND Lo_age THEN Age_err $=\left(H H_{-}\right.$age-Lo_age $) / 2$

19044 END If

19050 !

19056 SUBEND

19062 !

19068 !

19074 Concordia: SUB Concordia

19080 OPIIOK BASE 1

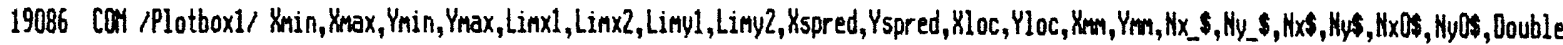

19092 COA /Plotboxla/ Lx1, Lx2, Ly1,Ly2, Offset

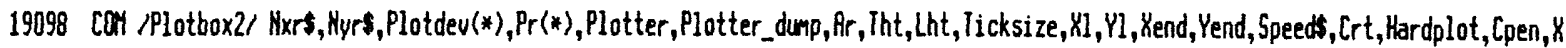
gu, Ygu

19104 Cor /Concordia/ Age_min, Age_nax, Ctick, oblique_tick

19110 off $\mathrm{XBD}$

19116 OFT KEY

19122 !

19128 If NOT Oblique_tick Then $C t s=" 0^{*}$

19134 If Oblique_tick THEN Cts $==^{-*}$

19140 Ctht $=$ Tht $/ 2$

19146 CRLL Tick(Age_nax-Rge_nin, Ctick)

19152 If Age_max-Age_min $>3000$ theN Ctick=Ctick/2 ! more ticks for large age-spans 
19158 If (Age_max-Age_min)/Ctick/8 THEN Ctick=Ctick/2

19164 Plot_concordia:K=8*Ctick

19170 Cht=1.05*Tht ! character-size for concordia-tick labels

19176 !

19182 ! Find where to put first tick for concordia curve

$19188 \quad C=0$

19194 FOR Pass=1 TO 2

19200 Rge_nin $=C$

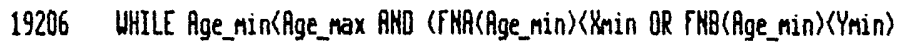

19212 Age_min=Rge_nintK

19218 END UHILE

19224 !

19230 If Pass=1 THEN

19236 C= Rge_nin-K

$19242 \quad k=C$ tick

19248 END IF

19254 KEXT Pass

$19260 \quad$ !

19266 FOR $D x=0$ io Double

19272 If Hardplot AND DX THEN

19278 UIELPORT $L x 1, L \times 2, L y 1, L y 2$

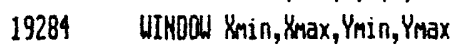

19290 END IF

19296 CLIP Xmin, Ymax, Ymin, Y Ymax

$19302 \quad 0=0$

19308 0[6

19314 FOR I= Rgge_nin-Ctick TO Age_max STCP Ctick/8! draw concordia curve

$19320 \quad X=F M A(T)$

$19326 \quad Y=F$ FB (T)

19332 If (X) X Xinin) AND (Y)YMin) THEN

19338 ORPHI X,Y

19344 [LSE

19350 MOUE $X, Y$

19356 END If

$19362 \quad$ !

19368 If $T / C$ tick $=$ INT(I/Ctick) THEN

19374 If T $>=$ Age_min THEN

$19380 !$

$19386 !$-..... Concordia ticks \& tick-labels -..-

19392 !

19398

19404

19410

19416

19422

19428

19434

19440

19446

19452

19458

19464

19470

19476

19482

19488

If $T$ THEK

If Oblique_tick THEN

I tangent to concordia for $T$, corrected for plot distortion Slope_angle=ATH(FNCslope $(T) * Y \mathrm{~m} / \mathrm{X} / \mathrm{m})$ lick_angle=Slope_angle-90! tick perpendicular to curve LOIR Tick_angle

END If

LORG 5

FOR L=1 TO $1+0 * *$ (Oblique_tick=0) !OISABLE LOOP

If Hardplot THEN IMOUE +FNCwidth(Ctht, $4 \mathrm{~mm}, 0,1,0) / 15$,-FNCheight $\left(C\right.$ tht, $\left.0, Y_{\mathrm{rm}}, 0\right) / 15$

CSIZE Ctht $*(4-L) / 3, .8+.2 * 0 b l i q u e$ tick

If $L=2$ THEN MOUE $X, Y$

LABEL Cts

NEXT L

If 0 THEK

! Check if tick-labels lie within plot-box $L=C h t *(1.5+I T T(L G T(T)))$

Nohars $t i c k=F M Z(T)+1$

If Hardplot THEN Length_tlabel $=(1.3 * 0 \mathrm{~b} l$ ique_tick $+1+N($ chars_tick $) * F N C w i d t h(C h t, 4 \times m, 0, A r, 0) !$ in WU

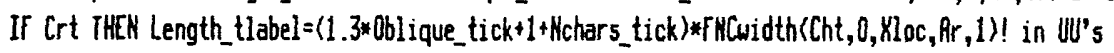


If Hardplot THEN Height_tlabel FNCheight $\left(C h t, 0, Y_{m}, 0\right) * 1.2$

\section{END If}

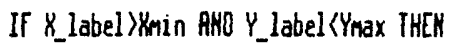
HOUE $x$-(Oblique_tick=0)*Cht*Ar/Xloc/2.,y LOR6 7+0blique_tick

csize Cht, Ar

If NOT Oblique_tick THEN LABEL FNZcons(I)

If Oblique_tick THEN LABEL FMZcons(T) $8^{*}$ "

\section{END If}

19620

19626

19632

19638

19644

ENB If

19650 NEXI I

19656 NEXT DX

19662 !

19668 If Files $="$ " THEN Errtype $=0$

19674 LDIR 0

19680 !

19686 SUBEND

19692 !

19698 !

19704 !

19710 R: UEC FNR(T) ! 207/235 ratio from age

19716 RETURN EXP(9.8485E-4*T)-1

19722 FNEND

19728 !

19734 B:DEF FHB(I)! 206/238 ratio from age

19740 RETURN EXP(1.55125[-4*])-1

19746 FNEND

$19752 !$

19758 Cslope:OEF FNCslope(I) ! Slope of concordia curve for time I

$19764 \quad L 8=1.55125 E-4$

$19770 \quad L 5=9.8485 E-4$

19776 RETURH LB*EXPi(L8-L5)*1)/L5

19782 FMEND

19788 !

19794 D:DEF FWO(X) I 207/235 age from ratio

19800 RETURN LOG $(1+\$) / 9.8485 E-4$

19806 FHEKO

$19812 !$

19818 E:DEF FNE(Y) ! 206/238 age frow ratio

19824 RETURN LOG $(1+4) / 1.55125[-4$

19830 FNEND

19836 !

19842 !

19848 Limi ts: SUB Limits (Xmin, Ymax, Ymin, Ymax, Rge_min, Rge__nax, Ytik_inter,Ytik_inter)

19854 ! Calculate $X$ - and $Y$-linits for age-defined concordia plot

19860 !

$19866 Y_{\max }=\mathrm{FH}$ H (Rge_nax)

$19872 Y_{\text {max }}=F$ HB $($ Age_nax $)$ 
19878 CALLL Tick(Yhax-FHAR(Age_min), Xtik_inter)

19884 CALL Tick(Ynax-FNB(Age_nin),Ytik_inter)

19890 !

$19996 \min =0$

19902 LOOP I calculate ninimum X-value for ninimum age

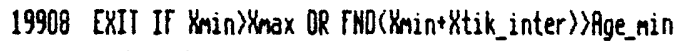

$19914 \quad X_{\text {min }}=X_{\text {min }}+$ Xtik_inter $_{2}$

19920 END LOOP

19926 !

19932 Ymin $=0$

19938 LOOP ! calculate minimun Y-value for minimur age

19949 EXIT If Ymin/Ymax OR FME(Ymin+2*Ytik_inter) ) Gge_min

$19950 Y_{\text {min }}=Y_{\text {min }}+Y_{\text {Y }}$ ik_inter

19956 END LOOP

19962 !

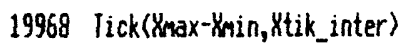

19974 Tick (Ynax-Ymin,Ytik_inter)

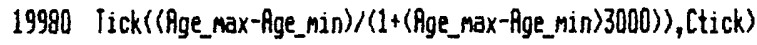

19986 !

19992 SUBENO

19998 !

20004 !

20010 Conc_inters:SUB Conc_inters(Slope, Intercept, Cint(*))

20016 OPIIOH BASE 1

20022 ! Calculate intercepts of a line with the concordia curve

20028 DIM Is (0:1)[5]

20034!

20040 FOR $J=0$ TO 1

20046 ! Ist Trial-T is $-500 \mathrm{Ma}$ (lower-int) or $5500 \mathrm{Ma}$ (upper)

20052 irial_t $t=-500+5500 * \mathrm{~J}$

20058 LOOP

20064 C=FNCslope(Trial_t)

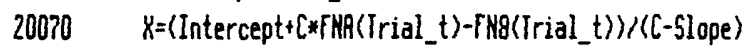

20076 If KK-1 THEN HO_inter

$20082 \quad !$

$20088 \quad T=F H D(X)$

20094 EXII If ABS(I-Irial_t $)<.01$

20100 Irial_t $t=1$

20106 END LOOP

$20112 \quad !$

$20118 \quad[\operatorname{int}(J+1)=0 R$ RONDO $(I, 6)$

20124 MEXI J

20130 SUBEXIT

20136 !

20142 No_inter:I\$(O)="LOULR"

20148 Is (1) = "UPPER"

20154 PRINT "YORKFII LINE HAS NO "QIS(J)\&" INTERCEPT WITH CONCORDIR..."

$20160 \operatorname{Cint}(J+1)=P I$

20166601020124

$20172 !$

20178 SUBEND

20184 !

$20190 !$

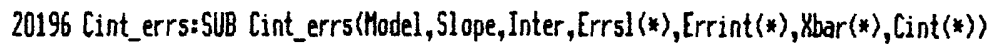

20202 ! Calculate concordia-intercept errors -.................

20208 !

20214 Slope_err=Errsl (Model)

20220 Inter_err=Errint(Hodel)

$20226 \quad X$ bar $=$ Xbar (Hodel)

20232 ! 
20238 FOR $J=3106$

20244 If J 35 AND Cint $(1)=P I$ THEN No_enu_inter

20250 If J $>4$ AND Cint(2) $=$ PI JHEN Ho_enu_inter

20256 SELECT J

20262 CASE 3 ! Lower intercept, younger age-limit

20268 Trial_ $t=-1000$

20274 CASE 4 ! Louer intercept, older age-limit

20280 Trial_t $t=[$ int (1)

20286 CASE 5 ! Upper intercept, younger age-linit

20292 Trial_t $t=[$ int (2)

20298 CASE 6 ! Upper intercept, older age-linit

20304 Trial_t $=6000$

20310 END SELECT

$20316 \quad$ !

20322 A=2*THEven $(\mathrm{J})-1$

$20328 \quad I=9 .[+99$

20334 LOOP

20340 S=FNCslope(Trial_t)

$20346 \quad B=$ FNB(Trial_t $t)-S *$ FNR(Trial_t $t)$-Inter

$20352 \theta=2 *\left(B *\left(S-S I_{0 p e}\right)+X_{-}\right.$bar $\left.* S l_{\text {ope_err }} 2\right)$

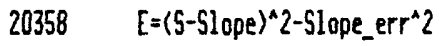

$20364 \quad G=0^{\wedge} 2-4 *\left[*\left(B^{\wedge} 2-\right.\right.$ Inter_err 2$)$

20370 If G<O THEN No_enU_inter

$20376 \quad X=(A \times S O R(6)-0)>(2 * E)$

20382 If $X(-1$ IHEN No_env_inter

$20388 \quad T=F N D(X)$

20394 If ABS(T-Trial_t) $)$ I THEN Ho_env_inter

20400 EXIT If RBS(I-Irial_t $)<.01$

20406 I $=$ ABS(T-Trial_t $)$

20412 Irial $t=T$

20418 END LDOP

20424 If $\operatorname{Cint}(J)\langle>P I$ THEM $\operatorname{Cint}(J)=$ DROUND(T,5)

20430 NEXT J

20436 Cint(7)=OROUKO(BBS(Cint(4)-Cint(3))/2,2)! average lower-int. error

$20442 \operatorname{Cint}(8)=0 R O U K D($ BQS $(C \operatorname{int}(6)-[\operatorname{int}(5)) / 2,2) !$ " upper-int.

20448 If $\operatorname{cint}(3)=P I$ OR $\operatorname{Cint}(4)=$ PI tHEN $\operatorname{Cint}(7)=$ PI

20454 If $\operatorname{Cint}(5)=$ PI $O R \operatorname{Cint}(6)=$ PI $T$ HEN $\operatorname{Cint}(8)=$ PI

20460 SUBEXIT

20466 !

20472 No_enu_inter:PRIMT "RRY"; J-2; "OF ERROR-ENUELOPE HAS MO COMCOROIA INTERCEPT..."

$20478 \operatorname{Cint}(\mathrm{J})=P \mathrm{PI}$

20484601020430

20490 !

20496 SUBEND

20502 !

20508 !

20514 Model4_calc:SUB Model4_calc(Slope, Inter, Cint(*), Inter $x y(*)$, Chordlength)

20520 CALL Conc_inters(Slope, Inter, Cint (*))

20526 Inter $x y(1,1)=F$ Hh(Cint(1)) I \& value of lower intercept

20532 Inter $x y(1,2)=F N B(C$ int $\{1)) \quad$ ! ${ }^{\prime \prime} " . "$

20538 Inter $x y(2,1)=F \operatorname{Nh}(\operatorname{Cint}(2))$ ! 8 " upper "

20544 Interxy $(2,2)=F$ N $B(\operatorname{Cint}(2)) \quad ! Y$

20550 Chordlength=SQR $\left(\left(\operatorname{Inter} x y\langle(2,1)-\text { Inter } x y(1,1))^{\wedge} 2+\langle\text { Interxy }(2,2)-\text { Inter } x y(1,2))^{\wedge} 2\right)\right.$

20556 SUBEND

20562 !

20568 !

20574 Help7:5U日 Help?

20580 OPIIOH BRSE 1

20586 OIH \$\$(13)[160]

20592 DATA"1: ACCEPT MODEL-1 Accept the MDOEL -1 fit as the best-fit line, so don't do any other nodel Yorkfits. s" $^{*}$ 
20598 DARA"2: DO MOOEL-2 Do a MODEL-2 fit to the data. A MODEL-2 fit weights each point equally, regardless of assigned erro rs. This nodel is useful"

20604 DATA "if the points are obviously scattered due to sone non-analytical cause, but you have no idea what that cause night be.s

20610 DARTA"

al to the degree"

6EMERRL MODEL -4 COMHENIS: A MODEL -4 fit assumes that the degree of scatter will increase proportion

20616 DATH of discordance either away fron the Upper Intercept (HOOEL-4UI) or the Lower Intercept (MODEL-4LI). So the HODEL-4UI fi $t$ weights the points

20622 DHTA "that are closest to the UPPER intercept the most, and HOOEL-4LI weights the points closest to the LOWER intercept the " ost. These nodels are"

20628 DATA"useful if you can assume that the most-concordant points are the least affected by complexities either in multi-episodic disturbance (4UI), or"

20634 DATA "inheritance of zircons with different 207/206 ages (4LI). SThe penalty you pay for choosing a MODEL - -1 fit is that the St udent's-t nul tiplier"

20640 DAIh "on the errors tends to be significantly higher, since except for the 2 nost-concordant points, the points only count as

'fractional' points"

20646 DATh "towards increasing the degrees of freedon of your data set."

20652 DATA "\$(Note: MOOEL-4 fits are only allowed with 6 or more points)\$"

20658 DATR 3: DD MODEL-4UI DO a MODEL-4UI fit to the data.s

20664 DATA 4: DO MODEL-4LI Do a MODEL-4LI fit to the data.

20670 READ S\$(*)

20676 CALL Helpscreen(S\$(*),21)

20682 SUBEND

20688 !

20694!

20700 Print_inters:SUB Print_inters(Cint_r(*),Cint(*),Prntr)

20706 ! print out the concordia intercepts, naking sure that no extra significant figures are printed out.

20712 DคTh $1,1,1,1$

20718 READ $A 1, \mathrm{A2}, 01,02$

20724 MAT Cint_ $r=$ Cint

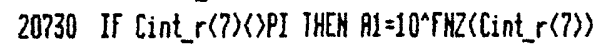

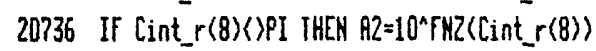

20242 If Cint_r(1)〈PI IHEN DI $=1$-FHZ(A1/Cint_r $r(1))$

20748 If Cint_r(2)<>PI THEN $02=1-$ FNZ(A2/Cint_r(2))

20754 If Cint_r(1)>PII IHEN Cint(1)=DROUNO(Cint_r $r(1), 01)$

20760 If Cint_r(2) (>PI THEN Cint(2)=DROUND(Cint_r(2),02)

20766 If Cint_r(1)-Cint(1))1 IHEN Cint(1)=INT(Cint_r(1))

20772 If Cint_r(2)-Cint(2))I THEN Cint (2) $=$ INT(Cint_r(2))

20778 PRINTER I5 Prntr

20784 PRINT

20790 If Cint_r(1)<>PI IHEN

20796 PRINT "LONER INTERCEPT: ";

20802 If Cint_r(1)()PI THEN PRINT Cint(1);

20808 If Cint_r(1)<>PI AND Cint_r(7)(PPI THEN PRINT "+/-";Cint(7);TRB(36); "Nla";

20814 If Cint_r(1)=PI OR Cint_r(7)=PI THEN PRINT TAB(36);" "lia";

20820 If Cint_r(3)=PI AND Cint_r(4)=PI THEN

20826 PRINT

20832 ELSE

20838 PRIKT TAB(53);"( ";

20844 ENO If

20850 If Cint_r(3)(PPI IHEN PRINT "> ";OROUNB(Cint(3),01);

20856 If Cint_r(4)(>PI IHEN PRINT " (";DROUND(Cint(4),01);")"

20862 If Cint_ $r(4)=$ PI THEN PRINT " $) "$

20868 END If

20874 If Cint_r(2)<>PI THEN

20880 PRINT "UPPER INTERCEPT: ";

20886 If Cint_r(2)<>PI THEN PRIHT Cint(2);

20892 If Cint_(2)<>PI AND Cint_r(8)<>PI THEN PRINT "+>-";Cint(8); TRB(36); "Ma";

20898 If Cint_(2)=PI OR Cint_r(8)=PI THEN PRINT TAB(36); "Na";

20904 If cint_(5)=PI AND Cint_r(6) $=$ PI THEN 


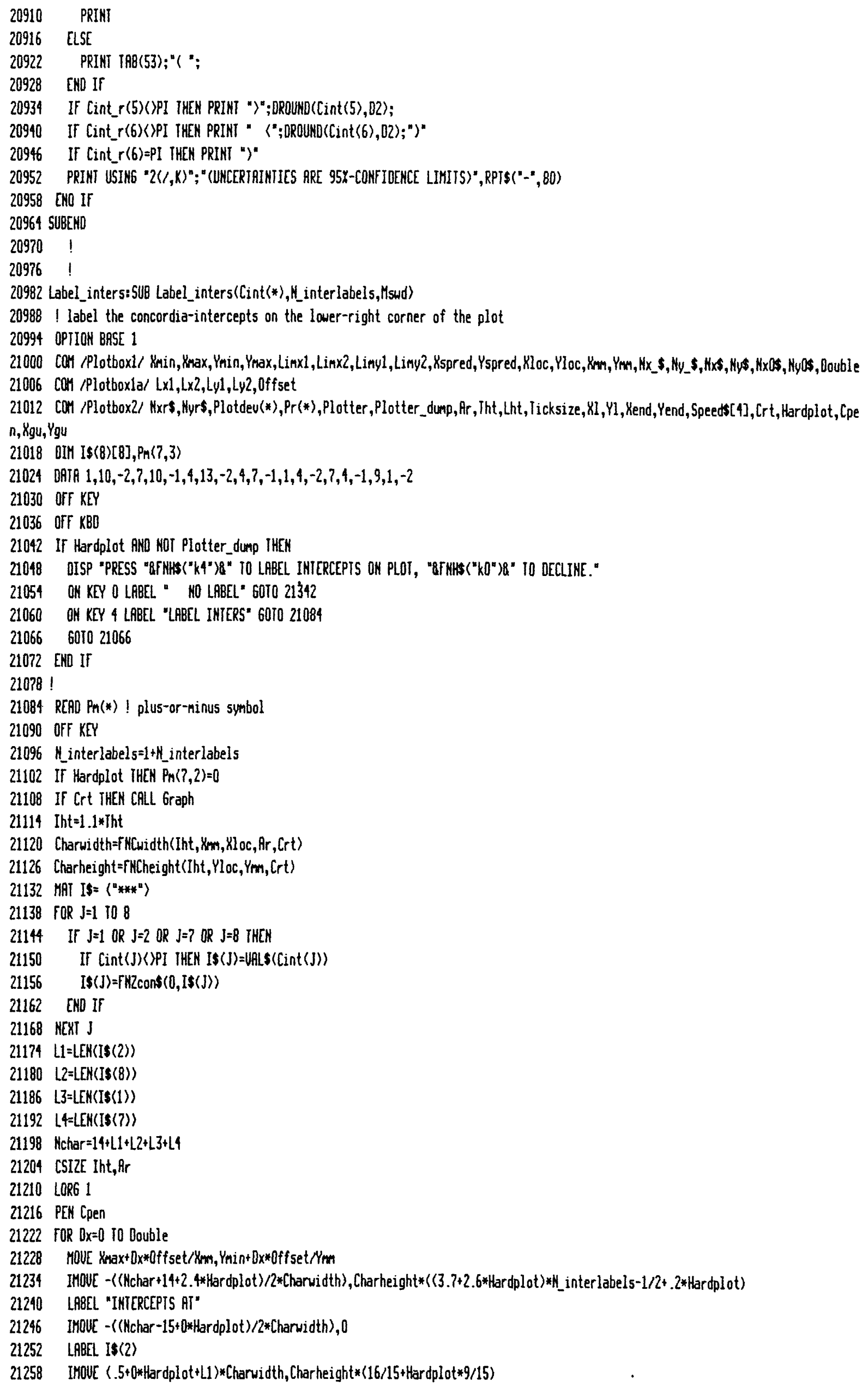


21264 SHMBOL PM(*)

21270 IMOUL $(1+0 *$ Hardplot $) *$ Charwidth $/ 2,0$

21276 LABELL Is (8)8" and "8Is(1)

21282 IMOUE $(12+13 * 5.5+0 *$ Hardplot $) *$ Charwidth, Charheight*(16/15+Hardplot*9/15)

21288 SMMBOL Pn(*)

21299 IMOUE $(1+0 *$ Hardplot $) *$ Charwidth $/ 2,0$

21300 LABEL Is $(7) 8^{*} \mathrm{Ma"}$

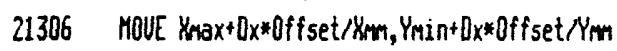

21312 IHoul ((-Nchar-12-2.9*Hardplot)/2*Charwidth), Charheight*((3.7*2.6*Hardplot) $) *$ _interlabel $5-2.8-1.3 *$ Hardplot $)$

21318 LABEL "(MSUD="BFHZCon\$ (DROUND(Msud,3))8")"

21324 NEXT $D X$

21330 MOUE $x_{\text {min, }} \gamma_{\text {max }}$

21336 PEN 0

21342 OISP

21348 SUBEND

21354 !

21360 !

21366 Graph:SUB Graph

21372 GRAPHICS ON

21378 ALPHA OFF

21384 SUBEND

21390 !

21396 !

21402 Rlpha: 5UB Alpha

21408 GRAPHTCS OfF

21414 ALPHA OK

21420 SUBEND

21426 !

21432 !

21438 Un:OEF FNUns (String\$)

21444 RETURN CHR\$(132)85tringst CHRS (128)

21450 FHEND

21456 !

21462 !

21468 Zcon:DEF FHZcons(U,OPIIONAt. 5\$) ! change variable to string, zeroes to O's

21474 DIM N\$5[80]

21480 IF NPRR $=1$ THEN

21486 ON ERROR $60 T 021558$

21492 US $=$ URL $\$(V)$

21498 OFF ERROR

21504 ELSE

$21510 \quad \$=5 \$$

21516 END IF

21522 FOR I=1 TO LEX(NS)

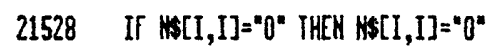

21534 NEXT I

21540 !

21546 RETURN *

21552 !

21558 RETURN " 0 "

21564 FNEND

21570 !

21576 !

21582 Ticklabel:SUB Ticklabel(Tick\$(*), Tickual (*), Dec(*), Dec_nax, Lzero, N_majortix)

21588 OPIIOK BASE 1

21594 ! convert the Iick-value array into a string array, with zeroes replaced by Oh's

$21600 !$

21606 DIM Zeros[10]

21612 Zeros $=" 0000000000 "$

21618 FOR I=1 TO H_majortix 
$21624 \operatorname{Tick} \$(I)=F H Z \operatorname{cons}($ Tickual $(I))$

21630 If Dec_nax AND Dec_nax $>0$ Cec(I) THEN

21636 If $\operatorname{Dec}(\mathrm{I})=0$ THEN $\operatorname{Ticks}(\mathrm{I})=\operatorname{Tick}(\mathrm{I}) \mathrm{s}^{\mathrm{a}}$."

$21642 T \operatorname{Tick} S(I)=T i c k s(I) 8 Z \operatorname{eros}[1, \operatorname{Dec} \max -\operatorname{Dec}(\mathrm{I})]$

21648 END If

21654 If Lzero THEN

21660 If Tickual(I)>O AND Tickual(I)《I THEN Tick\$(I)="0"8Iicks(I)

21666 If Tickual (I)<0 AND Tickual (I))-1 THEN Ticks(I) $=$ "-0"8 Ticks(I)[2]

21672 END If

21678 NEXI I

21684 SUBEND

21690 !

$21696 \quad$

21702 Cwidth:0EF FNCwidth(Charsize, thre, Xloc, Ar, Crt)

21708 ! return character width, in Uل's

21714 ffactor $=X 100 * \mathrm{Crt}+\mathrm{X}_{\mathrm{N}} *$ (NOT $\mathrm{Crt}$ )

21720 If Crt THEN RETURN Charsize*Ar//ffactor

21726 If HOT Crt THEN RETURN Charsize*Ar*1.183/.67/4factor

21732 FNEND

21738 !

21744 !

21750 Cheight:0EF FNCheight(Charsize,Yloc, Ym, Crt

21756 ! return character height in UU's

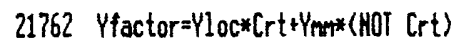

21768 RETURN Charsize/Nactor

21774 FMENO

$21780 !$

21786 !

21792 Printer_timeout: SUB Printer_timeout

21798 Cor /Printplot/ Printer_ok,Plotter_ok

21804 OFT IIMEOUT

21810 OFF KEY

21816 off KBD

21822 Printer_ok=0

21828 PRINTER IS CRT

21834 Clear

21840 PRINI TABXY(1,12);FNCenters(THHS ("*** PRINTER DOES NOT RESPOND ***"))

$21846 \operatorname{Beeper}(50,1200, .03, .01)$

21852 SUBEND

$21858 !$

21864 !

21870 Beeper:SUB Beeper(Ncycles, Hertz, Beep_time, Lait_time)

21876 FOR I=1 To Hcycles

21882 BEEP Hertz,Beep_time

21888 HAII Hait tine

21894 NEXI I

21900 SUBEND

21906 !

21912 !

21918 Plotter_timeout:SUB Plotter_timeout

21924 Con /Printplot/ Printer_ok,Plotter_ok

21930 OFF IIMEOUT

21936 Off KEY

21942 Off KBO

21948 Clear

21954 Plotter_ok=0

21960 PRIHT IABKY(1,12);FNCenters(FHHS("**** PLOITER GOES NOT RESPOND ****"))

$21966 \operatorname{Beeper}(50,1200, .03, .01)$

21972 SUBEND

21978 ! 
21990 Center:0EF FNCenters (\$\$) I create a centered string

21996 OIM Pad\$[80]

22002 L=LEN(SS)

22008 If L $\angle B O$ THEN

22014 Pads=RPTS(" ",(80-L)/2)

22020 RETURN PadshsshPads

22026 ELSE

22032 RETURN 5 S

22038 END If

22044 FNEND

$22050 !$

$22056 !$

22062 Check_rho: SUB Check_rho(Rho,_Yerr, Yerr, X,Y,Percent, Ok)

22068 If Percent THEN ! convert to either percent or fractional errors

22074 [x=kerr

$22080 \quad$ Ey=Yerr

22086 ELSE

$22092 \quad$ Ex=Rerr/4

$22098 \quad$ Ey=Yerr/Y

22104 ENO IF

$22110 !$

22116 If $A B S\left(R h_{0}\left\langle=1\right.\right.$ AND $\left(\left[x^{\wedge} 2+\left[y^{\wedge} 2\right)\right\rangle=\left(2 * R h_{0}\right) *\left[x_{*} * E y\right)\right.$ THEN

$221220 k=1$

22128 ELSE

$22134 \quad 0 \mathrm{k}=0$

22140 PRINT USING " $/, K^{n} ;$ FNH\$(" **** ERROR-CORREL. Of "QuRL\$(Rho)\&" IS NOT POSSIBLE **** ")

22146 Clunk

22152 UAIT 2

22158 END If

22164 SUBEND

22170 !

22176 !

22182 Col:0EF FNCol\$(K) ! return column-letter fron column-number

22188 If NK25 THEN RETURN CHPS $(\mathrm{N}+2+64$ )

22194 If H)=25 THEN RETURN "A"QCHRS $(H+2+64-26)$

22200 FNEND

$22206 !$

22212 !

22218 Convert_vc:5uB Convert_uc(Dfile, Uc $(*), \operatorname{Pr}(*)$, [Plot, Nanes $(*), 0 f_{-}$xnames, Df_ynames, Files, Mss $(*)$ )

22224 !

22230 ! Subprogran to access UISICALC/PF files. The UISICALC files must have

22236 ! the following attributes:

22242 !

22248 ! The file titie, if any, nust occupy the first row of the file, and

22254 ! will be printed out by this progran above the data.

$22260 !$

22266 ! The file must have been set for a column-width of 9 , and stored

22272 ! with a printer-uidth of 80 (not 132).

22278 !

22284 ! The column-nanes or headings can occupy 2 rows, but must appear dir-

22290 ! ectly above a line of "equals" signs (z=z:s=z=sz=s=) uhich serves as

22296 ! both an indicator of the column-heading rous and as a visual double-

22302 ! underscore sign.

22314 ! The sample or row names are assumed to be in the first two columns

$22320 !(A$ and $B)$ of the file.

22332 ! The file can contain up to 101 rous of sample-data and up to

22338 ! 50 columns. 
22350 OPIIOK BRSE 1

22356 OFF KEY

22362 Off KBD

22368 Alpha

22374 fileos $=$ Files

22380 !

22386 Cot/Printplot/ Printer_ok,Plotter_ok

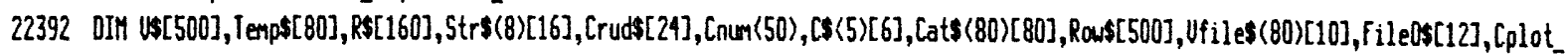
$\operatorname{col}(5)$, Ic(5), [p] $\$(5)[9]$

22398 DIM Titles[80],LLs5500],L2s[500], Ifiles[25], Top_cnames (50)[9], Botton_cnames (50)[9], Cnames (50)[18], Bh\$ (5) [9], it\$ (5) [9], Drive_s trings:[15], is[18]

22404 !

22410 Print $=0$

$22416 \quad$ Q $5=$ CHRS (34)

22422 Start:Clear

22428 OfF KEY

22434 ON KBD $60 T 022632$

22440 PRINIER IS CRT

$22446 !$

22452 PRITT TABXY(1,4); "Press "8FNHS(" kO ")6" for a catalog of the disk in the "gFNUns("left")\&"-hand drive."

22458 PRINT TABXY(1,6); "Press "8FNHS(" $k 1$ ") " for a catalog of the disk in the "afNUns ("right" ") "-hand drive."

22464 PRINT TABXY(1,8); "Press "8FWHS(" k2 ")8" or "BSTHHS(" CTRL H ")8" for HELP."

22470 PRINT TABXY(1,10); "Press "8FNHS(" k4 ")8" to load a Visicalc datafile into nemory."

22476 PRINT TABXY(1,14); "Press "8FFHHS(" $\mathrm{kg}$ ")8" to escape."

22482 !

22488 ON KEY 9 LABEL * ESCAPE" 601025140 I SUBENO line

22494 If Dfile THEN

22500 ON KEY 8 LABEL "SCRATCH FILE" 60TO Scratchfile

22506 PRINT TABXY(1,18); "(File "ifNUns(files):" currently in newory)"

22512 ELSE

22518 ON KEY 8 CRLL Clunk

22524 PRINT TABXY (1,18); "(No datafile currently in menory)"

22530 END IF

22536 If Dfile THEN ON KEY 8 LABEL "SCRATCH FILE" 60TO Scratchfile

22542 ON KEY 4 LABEL "LOAO ORTAFILE" GOTO Uhichfile

22518 ON KEY O LABEL "CATHLOG DrU "

22554 ON KEY 1 LABEL "CATHLOG Dru 11 " 601022884

22560 ON KEY 2 LABEL " HELP" 60TO 22679

22566 If Print THEN

22572 PRIHT IABXY(1,12); "Press "PFFHAS(" $\mathrm{k3}$ " 88 " to print out the datafile-data on the CRT."

22578 OH KEY 3 LABEL " DISPLAY OHTA" GOTO Use_crt

22584 DISP FNHS (" PRINTOUT OATh ON THE PRINTER (make sure printer is ON \& ON LINE)")

22590 ELSE

22596 PRINT TABXY(1,12); "Press "8FHHS(" $k 3$ ")\&" to print out the datafile-data on the printer."

22602 ON KEY 3 LABEL " PRINT DATA" 60TO Use_printer

22608 DISP FHHS (" DISPLAY DATA ON CRT OKLY ")

22614 END If

22620601022620

22626 !

$22632 k=F N 6 e t$ key

22638 off $\mathrm{KBO}$

22644 If $K=-79$ THEN DUMP RLPHA

22650 If K $>>8$ THEN

22656 Clunk

$22662 \quad 607022428$

22668 ELSE

22674 CRLL Help8

$22680 \quad 607022422$

22686 END If 
22698 Scratchfile: HAT $_{\mathrm{O}} U_{C}=(0)$

22704 MAT Hanes $=("$ ")

22710 Dfile $=0$

22716 OFF KEY

22722 Clear

22728 If Files $\langle$ )" IHEN

22734 DISP FHHS(" FILE "BCHRS(34)8Files\&CHRS (34)8" SCRATCHEO FROH MEMORY")

22740 Files $=" *$

22746 uAII 2

22752 END If

22758 SUBEXIT

22764 !

22770 Use_printer:Printer_ok=1

22776 ON IIMEOUT 7,1 CRLL Printer_timeout

22782 PRINTER IS 701

22788 PRINT

22794 If Printer_ok THEN

22800 OFT TIMEOUT

22806 Print $=1$

22812 BEEP

22818 END If

228246010 Start

22830 !

22836 Use_crt:Print=0

22842 BEEP

228486010 Start

22854 !

22860 Brive_string\$न\#/s\$(0)

22866 Brive_number=0

22872601022896

22878 !

22884 Drive_string\$=-1s\$ $\$ 1\rangle$

22890 Orive_number $=1$

22896 off $\mathrm{KEY}$

22902 Off KBD

22908 Clear

22914 DISP "Getting disk catalog..."

22920 FOR $P=1$ 10 1 I+Print

22926 If $P=1$ THEN PRINTER IS CRT

22932 If $P=2$ THEN PRIMTER IS 701

22938 ON ERROR GOTO Bad_cat

$22944 \quad S=8$ ! CAT strings containing filenanes start on 8th CAT string

22950 Nfiles $=0$

22956 CAT Drive_strings to Cats $(*)$

22962 DI5P

22968 OFF ERROR

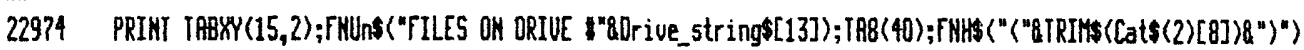

22980 PRINT

22986 LOOP ! deternine if file is a UISICALC /PF file (nust end in $A$ and be padded with underscore characters

22992 Tacs $=\operatorname{TRIH}(\operatorname{REUs}(\operatorname{Cat} \$(5)[1,10]))$

22998 EXIT IF LEM(Tacs) $=0$

23004 LOOP

23010 EXII If Tac $\$[1,1]()^{*}, "$

23016 Tacs=Tac\$[2]

23022 END LOOP

23028 If Tacs[1,1]="A" AND Nfiles $<=80$ THEN

$23034 \quad$ Nfiles $=1+$ Hfiles

23040 Ufiles(Hfiles)=REus(Tac $\$[2])$

23046 I display valid Visicalc files in inverse-video, non-yisicalc files normal \& in parentheses 


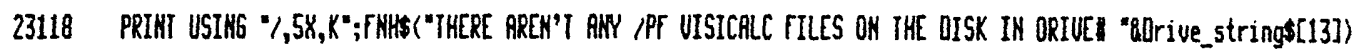

23124 DISP "Press"\&fNCto "when ready..."

23130 PAUSE

23136 G0I0 22422

23142 ELSE

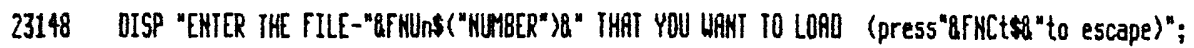

23154 Filenum $=0$

23160 INPUT filenum

23166 If Filenum $\{1$ OR Filenum $>$ Nfiles THEN 22422

23172 Temps=Ufiles(filenum)

231786010 Catfile

23184 END If

$23190 !$

23196 Bad_cat:OFF ERROR

23202 OISP FHHS(ERRN)

23208 Clunk

23214 UAIT 2

23220601022422

23226 !

23232 thichfile:Clear

23238 OFF KBD

23244 OFF KEY

23250 PRINT USING "2/,K,I,K,/"; "UHICH UISICALC ASCII-FILE [:ORIUE] DO YOU URHT TO BRING INTO MEMORY?","(example: Irjalfile:1)"

23256 If LEH(Files) THEN

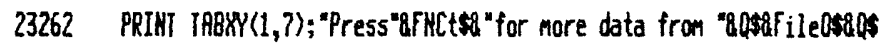

23268 Temps $=$ Files

23274 ENO If

23280 OH KBO 601023310

23286 ON KEY 9 LABEL " ESCAPE" GOTO 22422

23292 DISP "?"

23298601023298

23304 !

$23310 \quad K=$ FHGet_key

23316 off KBO

23322 OFf KEY

23328 SELECT $K$

23334 CASE 65 TO 90,97 TO 122

23340 BUTPUI KBD;CHR\$(K);

23316 PRINT TABXY $(1,7) ;$ RPTS(" ",80)

23352 OISP FHAst\$;

23358 INPUT Tenps

23364 If Temps $=$ "* THEN 22422

23370 CASE $-6 ?$ ! CONIINUE

23376 CASE ELSE

23382 G010 23280

23388 EHO SELECT

23394 Catfile:Clear

23400 L=LEN(Tenps)

23406 If $L=0$ THEN Whichfile! ENTER pressed with no input 
23412 If $L / 3$ THEN

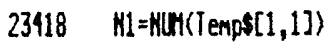

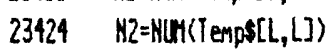

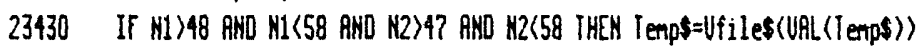

23436 END If

23442 LOOP

23448 Colon=PoS〈Tenps, ":"〉 ! Use colon to separate file-name fron drivel

23454 EXIT If Colon

23460 Temps=TRIMs(Temps)Q": "Quals(Drive_number)

23466 END LOOP

23472 ON ERROR 60TO Badspec

23478 Files $=$ TRIMS(Tenps[1, Colon-1])

23494 file0s=files

23490 L=LEN(Files)

23496 If L L10 THEN file $\$=$ file $\$ 8$ " $\mathrm{A}$ "

23502 Drive_number $=U$ ULL (Temp $\$[1+[0 l o n])$

23508 Orive_strings=Hs\$(Drive_number)

23514 OFf ERROR

$23520 !$

23526 L=LEN(Files)

23532 If L(10 THEN files[L+1,10]=RPTS(" ", 10-L) ! add underscore characters as fill to be compatible with the Visicalc format

$23538 \quad !$

23544 Badcount $=0$

23550 Recoup: ON ERROR GOTO Badfile

23556 DISP "Accessing file "8058file058058"..."

23562 If illes=files\&olive_string\$

23568 ASSIGN QP1 TO Ifiles

23574 ENTER EP1; US

23580 L=LEN(U)

$23586 L=4 I N(80, L)$

23592 Titles $=u s[1, L]$ ! first line in the file assumed to be a title-line

23598 files=ienps

23604 OFF ERROR

23610 !

23616 Clear

23622 !

23628 Controll=POS(Iitles, CHRS(27)) ! Escape-character location -

23634 If Controll THEN ! trin printer-control code if present

23640 Control2=pos(Titles[Control1]," "I! end of control-sequence

23646 If Control2 iHEN Titles=iitles[1, Control1-1]81itles[Contro12]

23652 END If

23658 PRINT USING "K, $/$ ";itlles

23664 OFF KBD

23670 605UB Search_colnanes

23676 !

23682 If HOT COOI THEN

23688 ! Enter the column-identifiers (alphabetic) and convert to colunn-numbers

23694 Enter_columns:DISP "EHTER THE COLUTHS $(0,6$, BF ...) CONTAINING \& [, Yerr], Y [, Yerr [, Rho(WY)]]";

23700 Enter coll: $: R 5=" *$

23706 MPT CnUm $=(0)$

23712 C_kbd: ON KBD GOTO 23742

23718 ON KEY 9 LPBEL " ESCPPE" 60TO Exit_sutb

23724 DISP "?"

$23730 \quad 607023730$

23736 !

$23742 \quad K=F N G e t$ key

23748 OfF KBD

23754 OfF $\mathrm{KEY}$

23760 If $K(65$ OR (K>90 PND K(97) OR K)122 THEN C_kbd

23766 OUTPUT KBD;CHRS(K); 
OISP FNAsts;

23778 Retrieve_str (Rs, Str\$(*), Hinputs)

23784 If Strs $(1)={ }^{*} *^{*}$ THEN Exit_sub

23790 MAT $c$ (s) (" " ")

$23796 \quad$

23802

23808

SELECT Hinputs

CASE 2

I $x$ - and Y-colutuns only

$23814 \quad \operatorname{cs}(1)=\operatorname{str} s(1)$

$23820 \quad[s(3)=5 \operatorname{st} s(2)$

23826 CRSE 4,5

$23832 \quad[s(1)=5 \operatorname{tr} s(1)$

$23838 \quad \operatorname{cs}(2)=5 \operatorname{tr} s(2)$

$23844 \quad \operatorname{cs}(3)=5 \operatorname{tr} s(3)$

$23850 \quad(s(4)=5 \operatorname{tr} s(4)$

$23856 \quad[\$(5)=S \operatorname{str}(5)$

23862 CASE ELSE

23868 CALL Bad_input("INURLIO COLUHW-INPUT")

238746070 Entercoll

23880 END SELECI

23886 ENO IF

$23892 !$

$23898 \quad !$

23904 FOR I=1 io 5 ! figure out where in the row-strings to find the numeric

23910 !

$23916 \quad L=\operatorname{LEN}(S S(I))$

23922 If L THEN

$23928 \quad \operatorname{cs}(\mathrm{I})=\mathrm{UPC}(\mathrm{Cs}(\mathrm{I})) \quad$ ! convert column-letters to uppercase

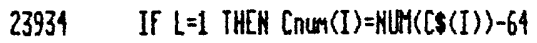

23940 ! Cnum(I) is the column-number for column-letter [s(I)

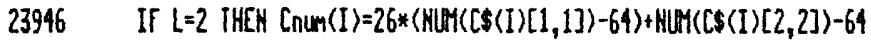

23952 IF L/2 THEN

23958 Bad_input(Cruds)

23964 G0T0 Entercoll

23970 END If

23976 If $((I=1$ OR I $=3$ ) AND Cnum(I)=0) OR Cnum(I) (3 OR Cnum(I)-2) Mum_datacolumns THEM

23982 Bad_input("INURLID COL UNN-SPECIFIER RESPOHSE")

$23988 \quad 6010$ Entercoll

23994 END IF

24000 END IF

24006 NEXI I

24012 !

24018 MAT $U_{c}=(0)$

24024 Row $=0$

24030 Lo0P I get sample names and numeric data from file

24036 ON ERROR GOTO Done

24042 Rous =" " I this row-string is the entire row of the file

24048 FOR I=1 TO Nsux ! build row-string from "Nsux" calls from file

24054 ENTER QP1; is

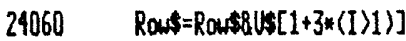

24066 MEXI I

24072 OFF ERROR

$24078 \quad R O N=1+$ Row

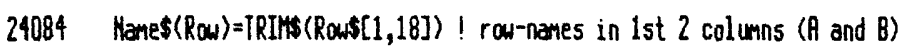

24090 FOR Col $=1$ i0 5

$24096 \quad C=$ Cnum(Col)

24102 If $C$ THEN

24108 OH ERROR $60 T 024132$ !**

$24114 \quad V_{C}($ Row, $[01)=V B L(R$ ow $5[9 *[-8,9 *[])$ ! extract numeric value

24120 0ISP Nanes (Rour), Rou; Col, Uc (Row, Col)

24126 ENO If 


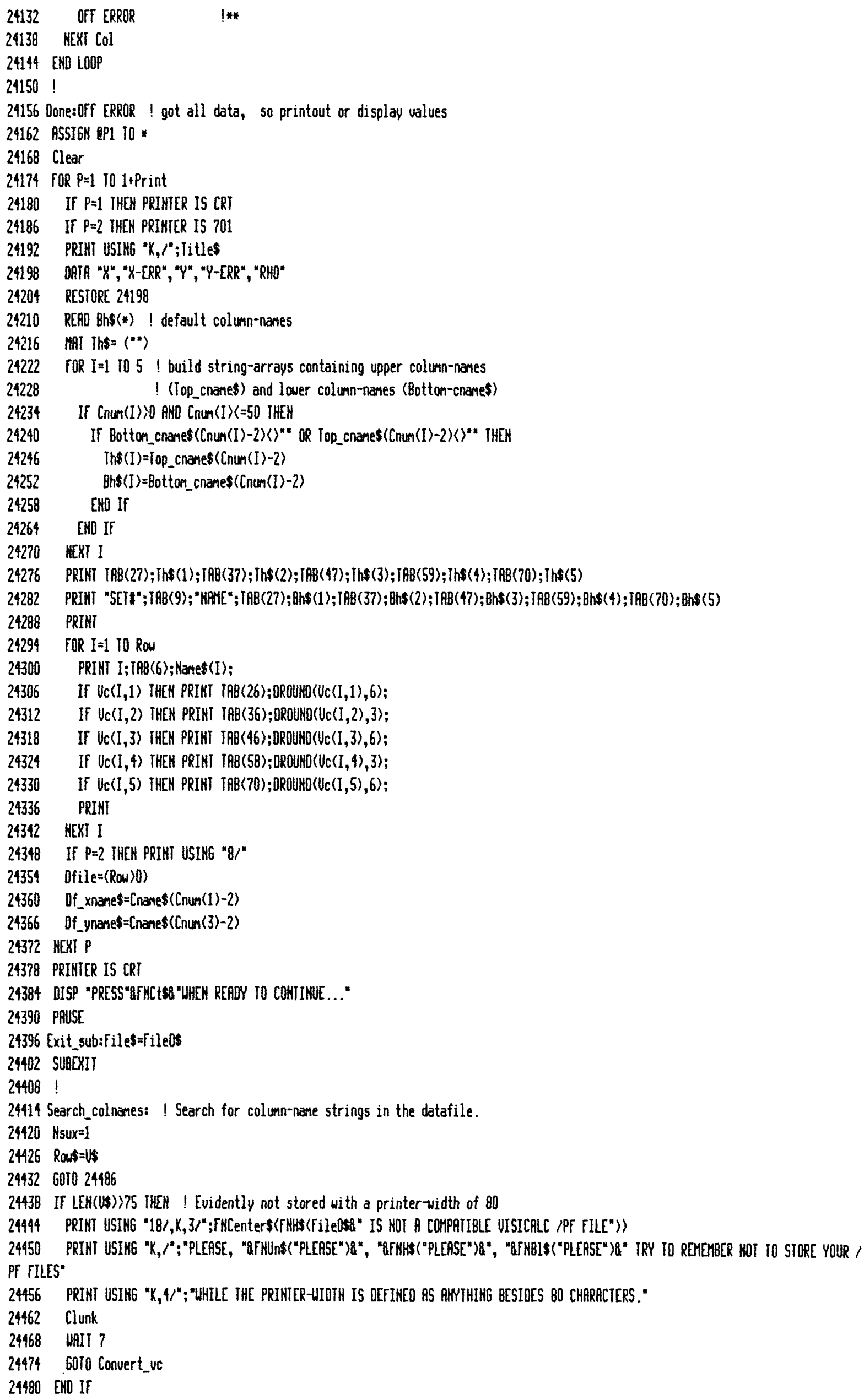


24886 If LEM(LS $)=72$ IHEN ! Length of 1 st string is 72 only if the real row

24492 LOOP ! is longer than 72 characters.

24498 ENTER RP1; US

24504 If LEN(us)<>72 THEN ! Sorry, really only $N * 8$ rows if still $=72$

$24510 \quad$ Hsux $=1+$ Hsux

$24516 \quad$ Rous =Roussius [4]! The operating system adds 3 space-characters after

24522 END IF

24528 EXIT If LEN(Us)<75 ! each 72 characters in the row.

24534 END LOOP

24540 ENO If

24546 Ncols=iNT(LEW(Rous)/9) I Assume a column-uidth of 9 characters.

$24552 !$

24558 ON ERROR GOTO No colnames ! Look for a row-string of repeating-equals

24564 LIs:" $\quad$ I signs extending across the whole file-width.

24570 L2S $=$ "n I This row separates the column-names from the

24576 LOOP ! actual data.

24582 Rous $="=$

24588 FOR I=1 IO Hsux

24594 ENTER EP1; US

24600 Rous $=$ Roussils $[1+3 *(I>1)]$

24606 MEXI I

24612 EXII If POS(Rous,RPIS("=",9*\#(cols))

$24618 \quad$ L2S $=$ L1S

$24624 \quad$ L1\$ $=$ Rows

24630 ENO LOOP

24636 OFF ERROR

24542 !

24648 ! extract column-names from the 2 rows above the "=sz=s ..." row.

24654 Nim_datacolumns=Hcols-2

24660 If Mur_datacolumns 22 JHEN

24666 Bad_input("FILE CONTAINS LESS IHAN THO OATR-COLLMNS")

24672 60T0 Convert_ve

24678 END If

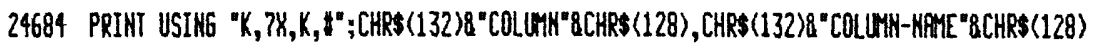

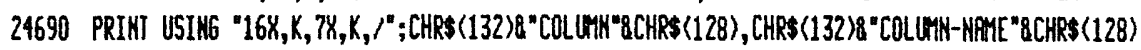

24696 FOR I $=1$ IO Mur_datacolumns

24702 ON ERROR GOTO 24714

24708 Top_cnanes $(I)=\operatorname{TRI}(L 25[9 *(I+1)+1,9 *(I+2)])$

24714 ON ERROR 6OTO 24732

29720 Botton_cnames $(\mathrm{I})=\operatorname{TRIH}(\mathrm{L} 1 \mathrm{~s}[9 *(\mathrm{I}+1)+1,9 *(\mathrm{I}+2)])$

24726 Cnames(I) $=$ Top_cnanes(I)8" "QBotton_cnanes(I)

24732 Off ERROR

24738 NEXT I

24744

24750 If Cplot THEN I Locate the correct columns if possible.

24756 MAT Cplot_col $=(0)$

$24762 \quad \mathrm{CoOl}=0$

24768 FOR I $=1$ TO Num datacolurns

24774 MAT $T C=(0)$

24780 TS=TRIHs(UPCs(Cnames(I)))

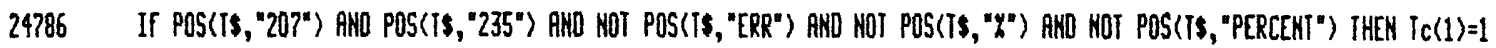

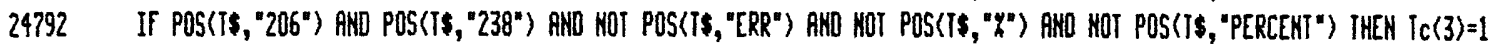

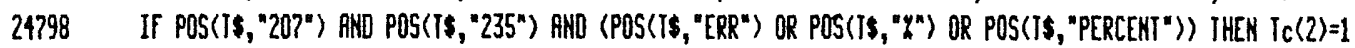

24804 If POS(IS,"206") AKD POS(I\$, "238") AND (POS(Is, "ERR") OR POS(Is, "z") OR POS(IS, "PERCENT")) THEN TC(4)=1

24810 If POS(Ts, "6/") AKD POS(IS, "8") ANO POS(IS, "7/") AHD POS(TS, "5") ANO POS(IS, "RHO") THEN CPlot_col(5)=I

$24816 \quad$ FOR J $=1$ IO 4

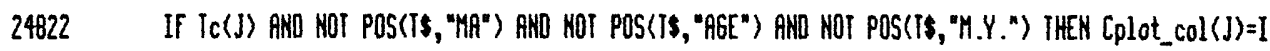

24828 MEXT J

24834 NEXT I

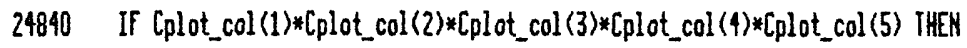


DATR "207/235", "7/5 ERROR", "206/238", "6/8 ERROR", "RHO"

24952

RESTORE 24846

24858

RLAO CP]s(*)

24864

FOR I $=1105$

24870

If Cplot_col(I)<=24 IHEN CS(I) $=$ CHRS $\left(C_{p} \operatorname{lot}_{-} \operatorname{col}(I)+66\right)$

24976

24882

24888

If Cplot_col(I))24 THEN CS(I) $=" A *$ RCHRS (Cplot_col(I) +40)

PRINT TAB(3);CS(I);TAB(14);CPIS(I)

\section{NEXI I}

Ccol=1

24900 END If

24906 END If

24912 !

24918 ! Printout the column-nanes in two vertical columns to avoid scrolling

24924 If NOT CoOl THEN

24930 Halfcols=IHT(Num_datacolumns/2)

24936 FOR I $=1$ TO Halfcols

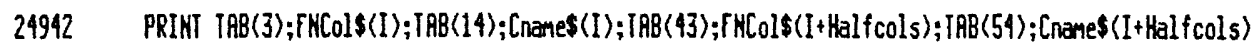

24948 NEXI I

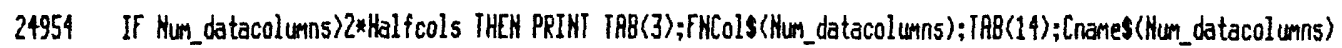

24960 END If

24966 RETURH

24972 !

24978

24984 Badfile:Badcount=1+Badcount! Assume that either the case or the drive-

24990 If Badcount/7 TheN I specifier is urong, \& keep trying until all

24996 ! pernutations of urong drives/case are tried.

25002 If Badcount=3 THEM Files:UPCS(Files)

25008 If Badcount $=5$ IHEN Files=Lucs(Files)

25014 Orive_strings[13,13]=UALS(HOT (URL(Drive_string $\$[13,13])$ ))

250206010 Recoup

25026 ENO If

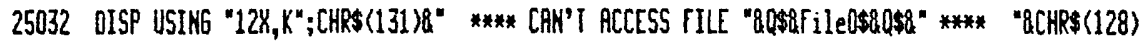

25038 files $=" *$

25044 Clunk

25050 UAIT 3

250566070 Convert_uc

25062 !

25068 Badspec:Bad_input("INURLIO FILE OR MASS-STORAGE SPECIfIER")

25074 riles="

250806070 thichfile

25086 !

25092 No_colnames:Clear

25098 OFF ERRDR

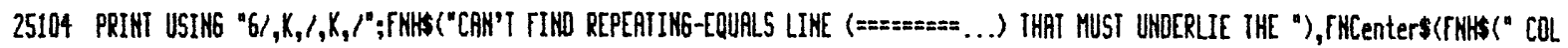
UHA-HFY[S "))

25110 Clunk

25116 UAII 5

25122 Clear

251286010 Convert_ve

$25134 \quad !$

25140 SUBEND

25146 !

25152 !

25158 Helpg: SUB Help8

25164 OPIION BASE 1

25170 OIM S\$(11)[160]

25176 DATA"4: LOAD DATPFILE Bring data from a UISICALC file on either drive into memory. The UISICALC file must conform to the following fornat: 1) It"

25182 0月Th "nust be a IPF file, 2) the column-width must be 9 characters, 3) sample-names nust appear in the first two columns, 4) a nane for the file" 
25188 DATR" should occupy the first row of the file, 5) the column names nust appear above the numeric data and be separated from $t$ he data by a"

25194 DATA"directly-underlying row of repeating equals- characters $\Leftrightarrow$, and (6) the file must have been stored with a printer-widt h of 80 , $\mathrm{NOT} 132 . "$

25200 DATA "So if you printed the file out on the printer with compressed type, be sure and restore the printer-width to 80 before storing the file."

25206 OATA" The column names can occupy either 1 or 2 rous.\$0: CATALOG Dru 10 Display a catalog of the files on the disk in the left-hand drive. $\$$

25212 DATA"1: CATALOf OrU 11 Display a catalog of the files on the disk in the right-hand drive. 5 "

25218 DAIA "6: PRINI DRTA Use the printer, rather than the CRT to print out the listing of the data obtained from the LOAD DA

TRFILE operation.\$"

25224. OATR"7: DISPLAN DRTA Use the CRT to show the data obtained from the LORD OATAFILE operation, rather than printing it out

on the printer . ${ }^{*}$

25230 DATR"B: SCRRTCH FILE Delete data fron any data-file currently in menory (but don't delete the data from the disk). Any $k$ eyboard- entered data will"

25236 DRTR "then be temporarily stored in merory as a set of datafile-data, and 50 can be re-used without typing the data in again.

\$"

25242 DATA $^{n}$ : ESCAPE Leave this function.

25248 !

25254 REAO S\$(*)

25260 CALL Helpscreen(S\$(*),21)

25266 SUBEND

25272 !

25278 !

25284 Bl:OEF FHBI\$(SS)

25290 RETURN CHRS(131)Q" "QS50" "QCHRS(128)

25296 FHENO

25302 !

25308 Ct: DEF fNCts

25314 RETURH " "\&FFHS("CONTINUE")\&" "

25320 FNEND

$25326 !$

25332 !

25338 Helpscreen:SuB Helpscreen(Input_string\$(*), Indent)

25344 ! prints out a message on the CRT with linefeeds at appropriate breaks

25350 ! between words. Also inserts one linefeed for each $\$$ symbol, and in-

25356 ! dents each line after a linefeed by Indent spaces. Tricky, huh?

25362 !

25368 OIM Largestring\$[2400], Linestring\$[80], Substring\$[2400], Revstring\$[80]

25374

25380 Clear

25386 OfF KEY

25392 OFF KBD

25398 OFF KNOB

25404 PRINTER IS CRT

25410 GRAPHICS OFF

25416 Largestring\$="

25422 ! build a single large string out of the input-string array

25428 FOR I=1 TO SIZE(Input_string\$,1)

25434 Largestring\$=Largestring\$itRIM\$(Input_string\$(I))\&: "

25440 NEXT I

25446 Maxlen $=80$

25452 !

25458 PRINT USING " 16X,K, /";FHhs (" FUNCTIONS OF KEYS DEFINED OURING LRST OISPLAN: ")

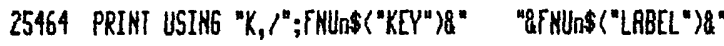

25470 Mlines $=5$ ! starting-line of HELP minus one.

25476 firstscreen=1 I First screen of this HELP?

25482 Firstline $=1$ ! Indicates not an indented line

25488 !

25494 LOOP 
EXII If LEN(TRIMs(Largestring\$)) $=0$

25506 Maxlen=80-Indent*(Firstline $=0)$

! Max. pernissible length, not

25512 Maxlen=HIN(haxlen,LEN(Largestrings))! including indentation.

25518 Lf=PoS(Largestrings, "s")

25524 If $L f=1$ THEN If first character is a linefeed token, then

25530 PRIMT I print a blank line \& strip the token.

25536 Mlines=1+Nlines ! Increrent linel counter.

25542 Firstlines! I Mext line not to be indented.

25548 If Largestrings: $=8$ " THEM Done

25554 Largestrings:Largestrings[2]! Strip linefeed token.

25560

ELSE

! first character not a linefeed token.

25566 Hext_lf=POS(Largestrings, "s") ! Position of next linefeed token.

25572 If Mext_lf iHEM I Substring to next linefeed token.

25578 Substring\$_Largestring\$[1, Hext_lf $f-1]$

25584 ELSE

25590 Substring\$:Largestrings

25596 END IF

25602 If LEN(Substring\$) (ftlaxlen THEN ! Don't need to find wordbreak.

25608 If Indent RMO MOI Firstline THEN PRINT IAB(Indent); ! Indent line.

25614 PRINT IRIRs(Substrings)

$25620 \quad$ Firstline $=0$

$25626 \quad$ Nlines=1+Hlines

25632 I Strip last printed line from total string.

25638 If LEW(Substring\$)<=LEN(Largestring\$) THEH Largestring\$=Largestring\$[1+LEN(Substring\$)]

25644 ELSE

25650 Linestrings=Largestrings $[1$, Maxlen] I fragment that can fit on line.

25656 Reustring\$=REus(Linestring\$) ! Reverse-string.

25662 REPERT I Don't include any linefeed tokens

25668 Lf=P0S(Reustrings, "s")

25624 If Lf THEN Reustrings=Reustring\$[2]

$25680 \quad$ UNIIL $L f=0$

25686 S=POS(Reustrings," ") ! find wordbreak position

25692 If $S$ THEN Reustrings=Reustrings[1+S] ! Goto wordbreak position.

25698 Lrevel.EM(Reustrings)

25704 If Indent AMO MOT Firstline IHEM PRIHT IAB(Indent); ! Indent line.

25710 PRINT TRIM(REUs(Reustring\$))

25716

25722

25728

25734

25740

25746

25752

25758

25764

25770

25776

Mlines $=1+$ Hlines

IIncrement linet-counter

If LEN(Largestring\$) $)=L$ rev THEN Largestrings:Largestrings[Lrevtl]

firstline $=0$

! Hext line will be indented.

ENO If

If Hlines $>16$ THEN I Pause for user to read.

OISP "Press"\&FHCtS\&" for nore HELP...';

IF NOT Firstscreen IHEN

OISP RPTS(CHRS(B),3)8", Roll screen with arrous"

ELSE

OISP

END If

25782 Firstscreen $=0$
25788 Nines $=0$

25794 PRUSE

25800 END IF

25806 END If

25812 ENO LOOP

$25818 \quad !$

25824 Done:0ISP "Roll screen with arrous, press"8fictsen"to return to nain program..."

25830 PAUSE

25836 SUBENO

25842 ! 
25848 !

25854 Students_t:DEF FuStudents_t(Hu)

25860 ! Student's-t approxination (fran Rndy Jurek for Mu $>=1$, KRL for less)

25866 If $H_{u}=0$ IHEH $H_{u}=1 . E-9$

25872 SELECT Hu

25878 CASE 1

25884 RETURN $12 . ?$

25890 CASE 11

$25896 \quad U=\| u-.85$

25902 RETURN $1.96-.00554568 / 59 R(W)+2.4059333 N+.6711777 / N^{\wedge} 2-.3831214 N^{\wedge} 3$

25908 CASE $<1$

25914 RETURH $1 /\left(-.00765 * H_{u+} .11085 * H_{u} \wedge 2-.02446 * H_{u} \wedge 3\right)$

25920 END SELECT

25926 FHEHD

25932 Rist: DEF FNAsts

25938 RETURH "(enter "QFHHS ("* $) 8$ " to escape)"

25944 THEYM

$25950 !$

25956 !

25962 Polycalc:SUB Polycalc(X(*),Y_in $(*)$, Coef $(*)$, Order, H)

25968 OPIION BASE 1

25974 ! Do actual calculations for a least-squares polynonial regression

25980 DIM $X_{a}(5,200), X_{b}(5,5), X_{d}(5,200), P_{0} y_{-}$array $(200,5), X_{c}(5,5), Y(200,1)$

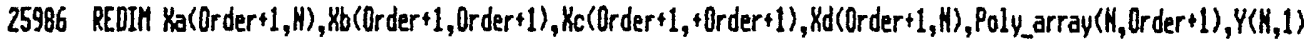

25992 ! Create Power natrix fron \&-walues

25998 FOR I $=1$ iO N

$26004 Y(I, 1)=\psi$ in $(I)$

26010 FOR $\mathrm{J}=1$ iO Order+1

$26016 \quad$ Poly_array $(I, J)=h(I)^{\wedge}(J-1)$

26022 NEXI J

26028 NEXT I

26034 !

26040 ! Solve natrix equations for best-fit polynomial coefficients

26046 NAT $K_{a}=$ TRN(Poly_array)

26052 MAT $x_{b}=Y_{a} \times P_{0}$ Ply $_{2} a r r a y$

26058 MAT $X_{c}=\operatorname{InU}\left(X_{b}\right)$

26064 HAT $X_{d}=X_{c} * X_{a}$

26070 HAT Coef $=X d * Y$

26076 SUBEND

$26082 !$

26088 Clear:SUB Clear

2609 OUTPUT KBD;Chrs (255)aChrs (25);

26100 SUBEMD

26106 !

26112 !

26118 Pbgrouth:SUB Pbgrouth

26124 ! Oraw a single-stage Pb-isotope grouth-curve if appropriate.

26130 OPIIOH BASE 1

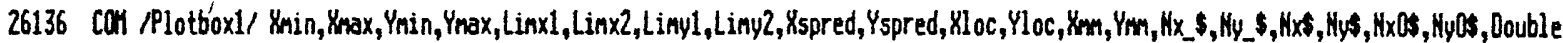

26142 cor /Plotboxla/ LX1, Lx2,Ly1,Ly2,0ffset

26148 CoH /Plotbox2/ Hxr\$, Hyr\$,Plotdeu(*),Pr(*),Plotter,Plotter_dump, Pr, Tht, Lht, Ticksize, X1, Y1, Xend, Yend, Speed, Crt, Hardplot, Cpen, X

gu, Ygu

26154 Car /Plotbox3/ Of_xnanes,Df_ynames,Dfile,Stack

26160 COI / 6 routh/ Lanbda $(0: 2), R O(0: 2), P(0: 2), E(0: 2)$

26166 oIH $2(0: 2), 4 \$(2)[7]$, Input $\$[80], \mathrm{Nu}(4)$

$26172 N \times s=[K Z \operatorname{con} \$(0, N \times s)$

26178 Hys $=F \mathrm{HZCOn} \$(0, H y)$

26184 If POS(Hxs, "206") AND POS(Nxs,"204") AND POS(Hy*,"207") AND POS(Hys,"204") IHEN Pbtype=1

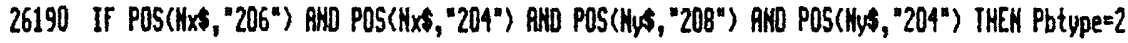

26196 If NOT Pbtype THEN SUBEEII 
26208 ОНTค $.155125 E-3, .98485 E-3, .049475\left[-3,11.152,12.998,31.230,1,137.88, .26455,3700,9.74, " 207 / 204^{\prime \prime}, 208 / 204 "\right.$

26214 RESTORE 26208

26220 RERO Lambda(*), RO(*),Z(*), $T 0, \mathrm{HuO}$, Ys(*)

26226 !

26232 Clear

26238 Alpha

26244 PRINT TABXY(1,11);"DO YOU UANT TO INCLUDE A SINGLE-STAGE GROUIH-CURUE IN IHE PLOT?"

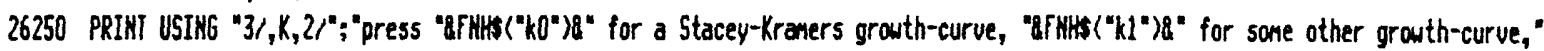

26256 PRINT "press "8FWHS (" $\mathrm{g}$ ") $)$ " to decline grouth-curve."

26262 ON KEY O LABEL "STACEY-KRPAERS" GOTO S_k

26268 OH KEY 1 LABEL " OTHER" GOTO Other

26274 OH KEY 9 LABEL " HO CURUE" GOTO 26892

26280601026280

26286 !

26292 Other: OFF KEY

26298 Clear

26304 PRINT IABXY $(1,10\rangle ;$;ENTER 4 URLUES TO DEFINE A SINGLE-STRGE Pb-ISOTOPE GROUTH CURUE:"

26310 PRINT TABYY (1,12);"206/204 at start of grouth, "QYS(Pbtype)Q" at start of grouth,"

26316 PRINT IABXY (1,13); "Tine before present to start grouth, and Mu (238/204) of source..."

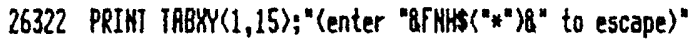

26328 Retrieve(Inputs, Hu(*), Hinputs)

26334 If TRIMs(Inputs) $=" *^{*}$ iHEN Pbgrowth

26340 If Minputs $\backslash>4$ THEN

26346 Bad_input( "HEED EXACTLY 4 MUMERIC URLUES FOR RESPONSE")

26352 60T0 Other

26358 END If

26364 If Hu(1)<=0 OR (Pbtype=1 AND Hu(2)<5) OR (Pbtype=2 AND Hu(2)<20) OR Hu(3)>6000 OR Hu(4)〈=0 THEN

26370 Bad_input("INURLID INPUT - CHECK HIMBERS \& IRY RGAIN.")

26376 60T0 other

26382 END If

$26388 R O(0)=H v(1)$

$26394 \mathrm{RO}(\mathrm{Pbtype})=\mathrm{Hu}(2)$

$2640010=H u(3)$

26406 MublHu(4)

26412 If Pbtype=2 THEN

26418 Clear

26424 INPUT "Enter the present-day 232/238 of the source...", Thu

26430 If Thus $=0$ THEN SUBEXII

$264362(2)=1 /$ Thu

26442 END IF

26448 !

$26454 S_{-} k: 0 F F \mathrm{KEY}$

26460 Oraw_grouthcurviClear

26466 PEN Cpen

26472 FOR I $=0102$

$26478\left[(\mathrm{I})=\mathrm{EXXP}_{\mathrm{N}}(\mathrm{Lanbda}(\mathrm{I}) * T 0)\right.$

$26484 P(\mathrm{I})=\mathrm{H}(\mathrm{W} / 2(\mathrm{I})$

26490 MEXI I

26496 Rige_min=0

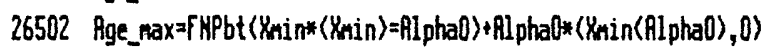

26508 Tick (Age_nax-Hge_nin, Tik)

$26514 k=8 * 7 \mathrm{ik}$

26520 Pht $=$ Tht $*(1-$ Hardplot/5)

$26526 \quad D=0$

26532 Ptht=Tht $/ 2.5$

26538 C $=10^{\wedge} \mathrm{FHz}(10)$

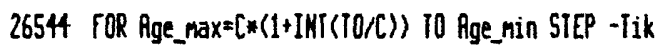

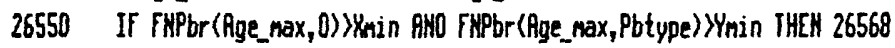

26556 NEXI Age_max 
26562 ! Avoid crouding of tick-labels

26568 Curvex_nin=FMPbr (HIH (Rge_nax, 10$), 0)$

26574 Curvex_nax=FNPbr (HAX(Age_min, 0$), 0$ )

$26580 \mathrm{f}=($ Curvex_nax-Curvex_nin)/3spred

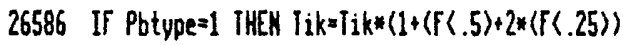

26592 !

26598 Graph

26604 FOR Dx=0 to Double

26610 If Hardplot AND Dx IHEN

26616 UIEUPORT $L x 1$, LxZ,LyI, LyZ

26622 WIHoOl Ynin, $Y_{\text {max }}, Y_{\min }, Y_{\text {max }}$

26628 END IF

26634 CLIP Xmin, Mnax $_{\text {, }}$ Mmin, $_{\text {, }}$ max

$26640 \quad 0=0$

26646 FOR T=Rge_max+2*Tik IO Age_min STEP -Tik/8 I Oraw grouth-curve

$26652 \quad K=F \mathrm{HPbr}(T, 0) \quad ! 206 / 204$

$26658 \quad Y=F \mathrm{Pbr}(1, \mathrm{Pbt}$ type $) \quad$ ! 207/204 or 208/204

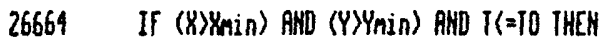

26670 DRPA $X, Y$

26676 ELSE

26682 MOUE $X, Y$

26688 END If

26694 !

26700 If DROUND(T/Tik,9)=INT(T/Tik) AND I $\langle=T O$ THEN

26706 If $\mathrm{T}\rangle=$ Rge_nin THEN

26712 !

26718 ! -.-.- gouth-curve ticks \& tick-labels -...-

26724 !

26730

26736

26742

26748

26754

26760

26766

26772

26778

26784

26790

26796

26802

26808

26814

26820

26826

26832

26838

26844

26850

26856

26862

26868

26874

LOR6 5

If Hardplot THEN IHOUE +FNCuidth(Ptht, $\left.x_{m}, 0,1,0\right) / 15$, FNCheight(Ptht, $\left.0,4 \mathrm{~m}, 0\right) / 15$

CSIZE Ptht, .8

LABEL "O"

If O THEN

! Check if tick-labels lie within plot-box

$L=P h t *(1.5+\operatorname{INT}(L \mathrm{LT}(T+(T=0))))$

Nchars_tick=FHZ(T)+1

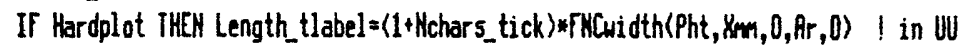

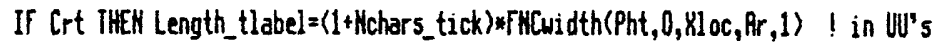

If Hardplot iHEN Height_tlabel =5NCheight $(P h t, 0, Y \mathrm{~m}, 0) \times 1.2$

If Crt THEN Height_tlabel=Pht/Nloc*11/15*1.2 ! in 6U's,m

$X$ _label=8-Length_tlabel

$Y$ _label=Y+Height_tlabel

If X_label) $X_{\text {min }}$ AnD Y_label(Y Max THEN

noue $x$-Pht*Ar/XIoc/7.,Y

LORG ?

CSIZE Pht, Br

LABEL FHZCons(T)

END If

END If

HOUE $X, Y$

END IF

DENOT (D)

26880 NEXTT T

26886 MEXT DX

26892 SUBEND

26898 !

26904 Pbr:DEF FMPbr(I,I) I Pb-isotope ratio fron age, assuming single-stage

26910 Con /Grouth/ Lambda(*),RO(*),P(*),[(*)

26916 RETURN RO(I)+P(I)*(E(I)-EXP(Lanbda(I)*I])) 
26922 FNENO

269281

26934 Pbt:DEF FHPbt(R,I) I Rge from Pb-isotope ratio, assuming single-stage

26940 CON / Grouth/ Lambda $(*), R O(*), P(*), E(*)$

26946 RETURY LOG(E(I)-(R-RO(I))/P(I))/Lambda(I)

26952 FNEND

26958 !

$26964 !$

26970 Err_env:SUB Err_enu(Slope, Slope_err, Inter, Inter_err, Xbar, Ymin, Ymax, Ymin, Ynax)

26976 ! Plot error-enuelope for a least-squares line

26982 OfF KEY

26988 OFF KBD

26994 FOR Arn=-1 101 STEP 2

$27000 \quad Y=1 . E-99$

$27006 \quad x=1 . E-99$

27012 Started $=0$

27018 UHILE XCXmax AHD YKYmax

27024 FOR $X=X_{\text {min }}$ to $Y_{\text {max }}$ STEP $\left(Y_{\max }-X_{\text {min }}\right) / 200$

27030 Ysqr_incr=Inter_err ${ }^{\wedge}+$ Slope_err ${ }^{\wedge} 2 * X *(X-2 *$ Wbar $)$

$27036 \quad Y_{s q}$ _incr $\left.=Y_{\text {sq__incr* }}\left(Y_{\text {sqr_incr }}\right\rangle 0\right)$

22042 If Ysqr_incr THEX

$27048 \quad Y=$ Slope* $X_{+}$Inter+Arm*SQR(Ysq_incr)

27054 If Y)Ynin AMO X) X Mnin THEN Started=1

27060 If Started THEN

$27066 \quad$ ORRI $X, Y$

27072 ELSE

27078 HOUE $X, Y$

27084 END If

27090 EYD If

27096 NEXT X

27102 END UHILE

27108 VExT Arn

22114 PEN 0

27120 SUBEND

27126 Clunk:SUB Clunk ! Clunky sound to indicate error

27132 ! BEEP 250,.1 ! for quiet enviroments

27138 ! BEEP 100, .2

27144 FOR $\mathrm{J}=0$ IO 2000 STEP 200

27150 FOR I=1 TO I ! Better for a noisy enviromment

27156 BEEP 3500-J, .001

27162 NEXI I

27168 NEXT J

27174 SUBEND 